\title{
A POSTERIORI ERROR ESTIMATION FOR A PDE-CONSTRAINED OPTIMIZATION PROBLEM INVOLVING THE GENERALIZED OSEEN EQUATIONS.*
}

\author{
ALEJANDRO ALLENDES ${ }^{\dagger}$, ENRIQUE OTÁROLA $^{\ddagger}$, AND RICHARD RANKIN ${ }^{\S}$
}

\begin{abstract}
We derive globally reliable a posteriori error estimators for a linear-quadratic optimal control problem involving the generalized Oseen equations as state equations; control constraints are also considered. The corresponding local error indicators are locally efficient. The assumptions under which we perform the analysis are such that they can be satisfied for a wide variety of stabilized finite element methods as well as for standard finite element methods. When stabilized methods are considered, no a priori relation between the stabilization terms for the state and adjoint equations is required. If a lower bound for the inf-sup constant is available, a posteriori error estimators that are fully computable and provide guaranteed upper bounds on the norm of the error can be obtained. We illustrate the theory with numerical examples.
\end{abstract}

Key words. linear-quadratic optimal control problems; generalized Oseen equations; Brinkman equations; Stokes equations; a posteriori error estimators; stabilized adaptive finite element methods.

AMS subject classifications. 49K20, 49M25, 65K10, 65N15, 65N30, 65N50, 65Y20.

1. Introduction. In this work we shall be interested in the design and analysis of computable a posteriori error estimators for a linear-quadratic optimal control problem involving the generalized Oseen equations; control constraints are also considered. To make matters precise, let $\Omega \subset \mathbb{R}^{d}$, with $d \in\{2,3\}$, be an open and bounded polytopal domain with Lipschitz boundary $\partial \Omega$ and $\mathbf{f} \in L^{2}(\Omega)^{d}$. Given a regularization parameter $\vartheta>0$ and a desired state $\mathbf{y}_{\Omega} \in L^{2}(\Omega)^{d}$, we define

$$
J(\mathbf{y}, \mathbf{u})=\frac{1}{2}\left\|\mathbf{y}-\mathbf{y}_{\Omega}\right\|_{L^{2}(\Omega)^{d}}^{2}+\frac{\vartheta}{2}\|\mathbf{u}\|_{L^{2}(\Omega)^{d}}^{2}
$$

We will be interested in the following PDE-constrained optimization problem: Find

$$
\min J(\mathbf{y}, \mathbf{u})
$$

subject to the generalized Oseen equations

$$
\left\{\begin{aligned}
-\varepsilon \Delta \mathbf{y}+(\mathbf{c} \cdot \nabla) \mathbf{y}+\kappa \mathbf{y}+\nabla \mathbf{p} & =\mathbf{f}+\mathbf{u} & & \text { in } \Omega, \\
\nabla \cdot \mathbf{y} & =0 & & \text { in } \Omega, \\
\mathbf{y} & =\mathbf{0} & & \text { on } \partial \Omega,
\end{aligned}\right.
$$

and the control constraints

$$
\mathbf{a} \leq \mathbf{u} \leq \mathbf{b} \text { a.e. in } \Omega,
$$

with $\mathbf{a}, \mathbf{b} \in \mathbb{R}^{d}$ satisfying $\mathbf{a}<\mathbf{b}$; the previous vector inequalities being understood componentwise. In (1.3), $\varepsilon, \kappa \in \mathbb{R}$ and are such that $\varepsilon>0$ and $\kappa \geq 0$ and $\mathbf{c} \in \mathbf{W}^{1, \infty}(\Omega)$

${ }^{*}$ Funding: Alejandro Allendes was supported by CONICYT through FONDECYT project 1170579. Enrique Otárola was supported by CONICYT through FONDECYT project 3160201. Richard Rankin was partially supported by BASAL PFB03 CMM project, Universidad de Chile.

†Departamento de Matemática, Universidad Técnica Federico Santa María, Valparaíso, Chile (alejandro.allendes@usm.cl).

${ }^{\ddagger}$ Departamento de Matemática, Universidad Técnica Federico Santa María, Valparaíso, Chile (enrique.otarola@usm.cl).

$\S$ School of Mathematical Sciences, University of Nottingham Ningbo China, Ningbo, China (richard.rankin@nottingham.edu.cn). 
is a solenoidal field. The generalized Oseen equations describe the low-Reynoldsnumber flow in porous media in situations where velocity gradients are non-negligible; they provide a unified approach to model flows of viscous fluids in a cavity and a porous media. It is well-known that the following choices of the parameters $\mathbf{c}$ and $\kappa$ yield the following flow models:

$$
\left\{\begin{array}{cl}
\mathbf{c}=\mathbf{0}, \kappa=0 & :-\varepsilon \Delta \mathbf{y}+\nabla \mathrm{p} \quad \text { (Stokes), } \\
\mathbf{c}=\mathbf{0} & :-\varepsilon \Delta \mathbf{y}+\kappa \mathbf{y}+\nabla \mathrm{p} \quad \text { (Brinkman), } \\
\kappa=0 & :-\varepsilon \Delta \mathbf{y}+(\mathbf{c} \cdot \nabla) \mathbf{y}+\nabla \mathrm{p} \quad \text { (Oseen) }
\end{array}\right.
$$

Our analysis allows for these choices of $\mathbf{c}$ and $\kappa$. Consequently, we present a unified analysis for the Stokes, Brinkman, Oseen and generalized Oseen equations.

The design of numerical techniques for approximating the solution to (1.3) has two major difficulties: first, in view of the so-called inf-sup condition [23, 24], arbitrary finite element methods are not allowed, and second, considering standard finite element methods produces poor approximation results when convection-dominated regimes are considered [38]. In order to overcome such difficulties, a variety of finite element techniques have been proposed and analyzed in the literature: the family of stabilized finite element methods. We refer the reader to [38] for an extensive overview.

In the PDE-constrained optimization context, a usual alternative for approximating the solution to the optimal control problem (1.2)-(1.4) is based on the so-called optimize-then-discretize approach. This technique discretizes the associated optimality system: the state equations (1.3), the adjoint equations and a variational inequality that characterizes the optimal control $\overline{\mathbf{u}}$. Consequently, the difficulties presented in the discretization of (1.3) are also present in the numerical approximation of the solution to (1.2)-(1.4). In addition, (1.2)-(1.4) is intrinsically nonlinear and, if $\mathbf{c} \neq \mathbf{0}$, presents a crosswind phenomena; the convection field of the adjoint equations is the negative of the one appearing in (1.3). The latter further motives the development of an efficient solution technique that, in convection-dominated regimes, properly treats the oscillatory behaviors that occur when approximating $\overline{\mathbf{y}}$ and its adjoint variable $\overline{\mathbf{w}}$ and resolves interior or boundary layers exhibited by both variables. Failure to resolve boundary layers can pollute the numerical solution in the entire domain; see [25] for results involving the scalar version of (1.2)-(1.4). However, numerical schemes based only on stabilized techniques are not sufficient to approximate the solution to (1.2)-(1.4): in addition to the efficient resolution of either interior or boundary layers, some possible geometric singularities must be resolved. This motivates the methods that we will use in this work: stabilized adaptive finite element methods.

Adaptive finite element methods (AFEMs) are iterative methods that improve the quality of the finite element approximation to a partial differential equation (PDE) on the basis of an essential ingredient: an a posteriori error estimator. The a posteriori error analysis for standard finite element approximations of linear second-order elliptic boundary value problems has a solid foundation $[3,36,46]$. When stabilized approximations are considered, several estimators have been introduced and analyzed in the literature; see, for instance, [1, 5, 9, 43, 47]. However, the a posteriori error analysis for finite element approximations of constrained optimal control problems has not been fully developed. In view of their inherent nonlinear feature, which appears due to the control constraints, the analysis involves more arguments and technicalities. An attempt to unify the available results has been carried out recently in [28] where the authors derive an important relationship between the error in optimal control problems and estimators, that satisfy a set of suitable assumptions, for problems associated with the state and adjoint equations [28, Theorem 3.2]. 
In the current work, the assumptions under which we perform the analysis are such that they can be satisfied for a wide variety of stabilized finite element methods as well as for standard finite element methods. This includes using a different stabilization method to approximate the state equation from that used to approximate the adjoint equation. We derive a posteriori error estimators that are globally reliable. Moreover, if a lower bound for the inf-sup constant is available, we can obtain a posteriori error estimators that are fully computable and provide guaranteed upper bounds on the norm of the error. Consequently, the estimators can be used as a stopping criterion in adaptive algorithms. The local error indicators that can be used to adaptively refine the mesh are locally efficient. Furthermore, we observe that they can be used to efficiently resolve boundary layers.

The outline of this paper is as follows. In section 2 we introduce some terminology used throughout this work. In section 3 we study the optimal control problem (1.2)(1.4) and obtain the associated optimality system. In section 4 we give the general form of the finite element methods that we consider for approximating the solution to (1.2)-(1.4). The core of our work is section 5 , where we devise a family of a posteriori error estimators. Under suitable assumptions, we obtain abstract reliability results in section 5.1 and local efficiency of the corresponding error indicators in section 5.2. In section 6 we consider the estimators that we can obtain for a particular approximation method in more detail. Finally, in section 7 we present a series of numerical examples to illustrate the theory.

\section{Preliminaries.}

2.1. Notation. For a bounded domain $A \subset \mathbb{R}^{t}, t \in\{1,2,3\}, L^{2}(A)$ and $H^{1}(A)$ denote the standard Lebesgue and Sobolev spaces, respectively; $L_{0}^{2}(A)$ is the subspace of $L^{2}(A)$ containing functions with zero mean value on $A$, and $H_{0}^{1}(A)$ is the subspace of $H^{1}(A)$ containing functions whose trace is zero on $\partial A$. We use bold letters to denote the vector-valued counterparts of the aforementioned spaces and an extra under accent for their matrix-valued counterparts. For instance, for $d \in\{2,3\}$, we denote $\mathbf{L}^{2}(A)=L^{2}(A)^{d}$ and $\underset{\approx}{\boldsymbol{L}}(A)=L^{2}(A)^{d \times d}$.

We now proceed to define notation associated with the discretization of the domain. Let $\mathscr{T}=\{K\}$ be a conforming partition of $\bar{\Omega}$ into simplicial elements $K$ $[18,23]$. We assume that $\mathscr{T}$ is a member of a shape regular family of partitions. Let $\mathcal{F}$ denote the set of all element edges(2D)/faces(3D) and $\mathcal{F}_{I} \subset \mathcal{F}$ denote the set of interior edges $(2 \mathrm{D}) /$ faces $(3 \mathrm{D})$.

For an element $K \in \mathscr{T}$, let:

- $\mathbb{P}_{n}(K)$ denote the space of polynomials on $K$ of total degree at most $n$;

- $\mathcal{F}_{K} \subset \mathcal{F}$ denote the set containing the individual edges(2D)/faces(3D) of $K$;

- $h_{K}$ denote the diameter of $K$;

- $\boldsymbol{n}_{\gamma}^{K}$ denote the unit exterior normal vector to the edge(2D)/face(3D) $\gamma \in \mathcal{F}_{K}$. For an edge(2D)/face(3D) $\gamma \in \mathcal{F}$, let:

- $\mathbb{P}_{n}(\gamma)$ denote the space of polynomials on $\gamma$ of total degree at most $n$;

- $\Omega_{\gamma}=\left\{K \in \mathscr{T}: \gamma \in \mathcal{F}_{K}\right\}$

- $h_{\gamma}$ denote the diameter of the edge(2D)/face(3D) $\gamma$.

To simplify the exposition of the material, we define $\mathbf{V}=\boldsymbol{H}_{0}^{1}(\Omega)$ and $Q=L_{0}^{2}(\Omega)$ with norms $\|\cdot\|_{\mathbf{V}, \Omega}$ and $\|\cdot\|_{Q, \Omega}$ defined, for all $\boldsymbol{\xi} \in \mathbf{V}$ and $\phi \in Q$, by

$$
\|\boldsymbol{\xi}\|_{\mathbf{V}, \Omega}^{2}:=\sum_{K \in \mathscr{T}}\|\boldsymbol{\xi}\|_{\mathbf{V}, K}^{2} \text { and }\|\phi\|_{Q, \Omega}^{2}:=\sum_{K \in \mathscr{T}}\|\phi\|_{Q, K}^{2}
$$


where

$$
\|\boldsymbol{\xi}\|_{\mathbf{V}, K}^{2}:=\varepsilon\|\nabla \boldsymbol{\xi}\|_{\underset{L^{2}(K)}{2}}^{2}+\kappa\|\boldsymbol{\xi}\|_{L^{2}(K)}^{2} \text { and }\|\phi\|_{Q, K}^{2}:=\|\phi\|_{L^{2}(K)}^{2}
$$

The relation $a \lesssim b$ indicates that there exists a constant $C$ such that $a \leq C b$. The constant $C$ may be different at each occurrence but is independent of $a, b$ and the size of the elements in the mesh.

2.2. Inequalities. For $K \in \mathscr{T}$ and nonnegative integers $l$, we denote by $\Pi_{K, l}$ the $\boldsymbol{L}^{2}(K)$-orthogonal projection operator onto $\mathbb{P}_{l}(K)^{d}$. This operator is defined as

$$
\Pi_{K, l}: \boldsymbol{L}^{2}(K) \rightarrow \mathbb{P}_{l}(K)^{d}, \quad\left(\mathbf{t}-\Pi_{K, l}(\mathbf{t}), \mathbf{v}\right)_{L^{2}(K)}=0 \quad \forall \mathbf{v} \in \mathbb{P}_{l}(K)^{d}
$$

Throughout the manuscript we will frequently make use of the following inequalities. First, if $K \in \mathscr{T}$ and $\boldsymbol{\xi} \in \mathbf{V}$, we have the Poincaré inequalities [10, 33, 37]

$$
\|\boldsymbol{\xi}\|_{\boldsymbol{L}^{2}(\Omega)} \leq \mathrm{C}_{P, \Omega}\|\nabla \boldsymbol{\xi}\|_{\boldsymbol{\sim}^{2}(\Omega)} \text { and }\left\|\boldsymbol{\xi}-\Pi_{K, 0}(\boldsymbol{\xi})\right\|_{\boldsymbol{L}^{2}(K)} \leq \frac{h_{K}}{\pi}\|\nabla \boldsymbol{\xi}\|_{\boldsymbol{L}^{2}(K)}
$$

where

$$
\mathrm{C}_{P, \Omega}=\frac{1}{\pi}\left(\sum_{i=1}^{d} \frac{1}{\left|l_{i}\right|^{2}}\right)^{-1 / 2}
$$

with $\left|l_{1}\right|, \ldots,\left|l_{d}\right|$ being the sides of a $d$-dimensional box containing $\Omega$. We immediately comment that these inequalities imply that, for $\boldsymbol{\xi} \in \mathbf{V}$ and $K \in \mathscr{T}$,

$$
\|\boldsymbol{\xi}\|_{\boldsymbol{L}^{2}(\Omega)} \leq \mathrm{C}_{\Omega}\|\boldsymbol{\xi}\|_{\mathbf{V}, \Omega} \text { and }\left\|\boldsymbol{\xi}-\Pi_{K, 0}(\boldsymbol{\xi})\right\|_{\boldsymbol{L}^{2}(K)} \leq \mathrm{C}_{K}\|\boldsymbol{\xi}\|_{\mathbf{V}, K}
$$

where

$$
\mathrm{C}_{\Omega}= \begin{cases}\frac{\mathrm{C}_{P, \Omega}}{\sqrt{\varepsilon}}, & \text { if } \kappa=0 \\ \min \left\{\frac{\mathrm{C}_{P, \Omega}}{\sqrt{\varepsilon}}, \frac{1}{\sqrt{\kappa}}\right\}, & \text { if } \kappa \neq 0\end{cases}
$$

and

$$
\mathrm{C}_{K}= \begin{cases}\frac{h_{K}}{\pi \sqrt{\varepsilon}}, & \text { if } \kappa=0 \\ \min \left\{\frac{h_{K}}{\pi \sqrt{\varepsilon}}, \frac{1}{\sqrt{\kappa}}\right\}, & \text { if } \kappa \neq 0\end{cases}
$$

We define $\mathcal{A}: \mathbf{V} \times \mathbf{V} \rightarrow \mathbb{R}, \mathcal{B}: \mathbf{V} \times Q \rightarrow \mathbb{R}$ and $\mathcal{C}: \mathbf{V} \times \mathbf{V} \rightarrow \mathbb{R}$ by

$$
\left\{\begin{array}{l}
\mathcal{A}(\boldsymbol{\xi}, \boldsymbol{\zeta}):=\varepsilon(\nabla \boldsymbol{\xi}, \nabla \boldsymbol{\zeta})_{\underset{\approx}{\boldsymbol{L}^{2}}(\Omega)}+(\kappa \boldsymbol{\xi}+(\mathbf{c} \cdot \nabla) \boldsymbol{\xi}, \boldsymbol{\zeta})_{\boldsymbol{L}^{2}(\Omega)} \\
\mathcal{B}(\boldsymbol{\zeta}, \phi):=(\phi, \nabla \cdot \boldsymbol{\zeta})_{L^{2}(\Omega)} \\
\mathcal{C}(\boldsymbol{\xi}, \boldsymbol{\zeta}):=\varepsilon(\nabla \boldsymbol{\xi}, \nabla \boldsymbol{\zeta})_{\underset{\approx}{\boldsymbol{L}^{2}(\Omega)}}+(\kappa \boldsymbol{\xi}-(\mathbf{c} \cdot \nabla) \boldsymbol{\xi}, \boldsymbol{\zeta})_{\boldsymbol{L}^{2}(\Omega)}
\end{array}\right.
$$

The fact that $\mathbf{c}$ is a solenoidal vector field and integration by parts implies that

$$
\mathcal{A}(\boldsymbol{\xi}, \boldsymbol{\zeta})=\mathcal{C}(\boldsymbol{\zeta}, \boldsymbol{\xi}) \quad \forall \boldsymbol{\xi}, \boldsymbol{\zeta} \in \mathbf{V}
$$

Moreover, for all $\boldsymbol{\xi} \in \mathbf{V}$,

$$
\mathcal{A}(\boldsymbol{\xi}, \boldsymbol{\xi})=\mathcal{C}(\boldsymbol{\xi}, \boldsymbol{\xi})=\|\boldsymbol{\xi}\|_{\mathbf{V}, \Omega}^{2}
$$


and, for all $\boldsymbol{\xi}, \boldsymbol{\zeta} \in \mathbf{V}$,

$$
\mathcal{A}(\boldsymbol{\xi}, \boldsymbol{\zeta}) \leq \mathrm{C}_{\mathrm{ct}}\|\boldsymbol{\xi}\|_{\mathrm{V}, \Omega}\|\boldsymbol{\zeta}\|_{\mathrm{V}, \Omega}, \quad \mathcal{C}(\boldsymbol{\xi}, \boldsymbol{\zeta}) \leq \mathrm{C}_{\mathrm{ct}}\|\boldsymbol{\xi}\|_{\mathrm{V}, \Omega}\|\boldsymbol{\zeta}\|_{\mathrm{V}, \Omega}
$$

where

$$
\mathrm{C}_{\mathrm{ct}}=1+\frac{\mathrm{C}_{\Omega}}{\sqrt{\varepsilon}}\|\mathbf{c}\|_{L^{\infty}(\Omega)},
$$

with $\|\mathbf{| c |}\|_{L^{\infty}(\Omega)}$ being the $L^{\infty}(\Omega)$ norm of $|\mathbf{c}|$ and $\mathrm{C}_{\Omega}$ being given by (2.7).

We now recall the standard inf-sup condition [23, 24]: there exists a positive constant $\beta$ such that

$$
\beta\|\phi\|_{L^{2}(\Omega)} \leq \sup _{\boldsymbol{\xi} \in \mathbf{V} \backslash\{\mathbf{0}\}} \frac{\mathcal{B}(\boldsymbol{\xi}, \phi)}{\|\nabla \boldsymbol{\xi}\|_{\widetilde{\sim}^{2}(\Omega)}} \quad \forall \phi \in Q
$$

Notice that, in view of $\|\boldsymbol{\xi}\|_{\mathbf{V}, \Omega}^{2} \leq\left(\varepsilon+\kappa \mathrm{C}_{P, \Omega}^{2}\right)\|\nabla \boldsymbol{\xi}\|_{\underset{\sim}{\boldsymbol{\sim}^{2}(\Omega)}}^{2}$, we have that

$$
\|\phi\|_{Q, \Omega} \leq \mathrm{C}_{\text {is }} \sup _{\boldsymbol{\xi} \in \mathbf{V} \backslash\{\mathbf{0}\}} \frac{\mathcal{B}(\boldsymbol{\xi}, \phi)}{\|\boldsymbol{\xi}\|_{\mathbf{V}, \Omega}} \quad \forall \phi \in Q
$$

where

$$
\mathrm{C}_{\text {is }}=\frac{\sqrt{\varepsilon+\kappa \mathrm{C}_{P, \Omega}^{2}}}{\beta} .
$$

3. Optimal control problem: optimize. In this section we briefly analyze the optimal control problem (1.2)-(1.4). To accomplish this task, we begin by introducing the following weak version of the state equations (1.3): Find $(\mathbf{y}, \mathbf{p}) \in \mathbf{V} \times Q$ such that

$$
\left\{\begin{aligned}
\mathcal{A}(\mathbf{y}, \boldsymbol{\xi})-\mathcal{B}(\boldsymbol{\xi}, \mathrm{p}) & =(\mathbf{f}+\mathbf{u}, \boldsymbol{\xi})_{\boldsymbol{L}^{2}(\Omega)} & & \forall \boldsymbol{\xi} \in \mathbf{V}, \\
\mathcal{B}(\mathbf{y}, \phi) & =0 & & \forall \phi \in Q,
\end{aligned}\right.
$$

where the bilinear forms $\mathcal{A}$ and $\mathcal{B}$ are defined by (2.9) and we recall that $\varepsilon>0, \kappa \geq 0$, $\mathbf{c} \in \mathbf{W}^{1, \infty}(\Omega)$ is a solenoidal field, $\mathbf{f} \in \mathbf{L}^{2}(\Omega), \mathbf{V}=\boldsymbol{H}_{0}^{1}(\Omega)$ and $Q=\boldsymbol{L}_{0}^{2}(\Omega)$. In view of the fact that $\mathcal{A}$ satisfies (2.11) and (2.12) and $\mathcal{B}$ satisfies the inf-sup conditions (2.14) and (2.15), we conclude the well-posedness of problem (3.1) [23, 24]. We also mention that, due to de Rham's Theorem (see Section 4.1.3 and Theorem B73 in [23]), we can consider the following equivalent formulation of problem (3.1): Find $\mathbf{y} \in \mathbf{V}_{0}$ such that

$$
\mathcal{A}(\mathbf{y}, \boldsymbol{\xi})=(\mathbf{f}+\mathbf{u}, \boldsymbol{\xi})_{\boldsymbol{L}^{2}(\Omega)} \quad \forall \boldsymbol{\xi} \in \mathbf{V}_{0}
$$

where $\mathbf{V}_{0}:=\left\{\mathbf{v} \in \boldsymbol{H}_{0}^{1}(\Omega): \nabla \cdot \mathbf{v}=0\right\}$.

To analyze our optimal control problem, we follow [30,44] and introduce the socalled control to state map $S: \boldsymbol{L}^{2}(\Omega) \rightarrow \mathbf{V}_{0}$ which, given a control $\mathbf{u}$, associates to it the state $\mathbf{y}$ that solves (3.2). In addition, we define, for $\mathbf{a}, \mathbf{b} \in \mathbb{R}^{d}$ with $\mathbf{a}<\mathbf{b}$, the set

$$
\mathbf{U}_{a d}:=\left\{\mathbf{v} \in \mathbf{L}^{2}(\Omega): \mathbf{a} \leq \mathbf{v} \leq \mathbf{b} \quad \text { a.e. in } \Omega\right\} ;
$$

the vector inequalities being understood componentwise. The set $\mathbf{U}_{a d}$ is a bounded, convex, closed and nonempty subset of $\mathbf{L}^{2}(\Omega)$ and consequently weakly sequentially compact. Thus, in view of the fact that the reduced cost functional

$$
f(\mathbf{u}):=\frac{1}{2}\left\|S(\mathbf{u})-\mathbf{y}_{\Omega}\right\|_{\boldsymbol{L}^{2}(\Omega)}^{2}+\frac{\vartheta}{2}\|\mathbf{u}\|_{\boldsymbol{L}^{2}(\Omega)}^{2}
$$


is weakly lower semicontinuous and strictly convex $(\vartheta>0)$, we conclude the existence and uniqueness of an optimal control $\overline{\mathbf{u}}$ and an optimal state $\overline{\mathbf{y}}$ that satisfy (3.2), or equivalently (3.1); see Theorem 2.14 in [44]. The existence of $\bar{p}$ such that $(\overline{\mathbf{y}}, \overline{\mathbf{p}})$ solves (3.1) follows from de Rham's Theorem. In addition, we have that $\overline{\mathbf{u}}$ satisfies the first-order optimality condition

$$
f^{\prime}(\overline{\mathbf{u}})(\mathbf{u}-\overline{\mathbf{u}}) \geq 0 \quad \forall \mathbf{u} \in \mathbf{U}_{a d}
$$

see [44, Lemma 2.21]. To explore this variational inequality, and to obtain optimality conditions, we define, on the basis of the formal Lagrange method (see [21, Section $3.3]$ and [44, Section 2.10]), the adjoint state ( $\mathbf{w}, \mathbf{q})$ as the unique solution to the following weak problem: Find $(\mathbf{w}, \mathbf{q}) \in \mathbf{V} \times Q$ such that

$$
\left\{\begin{aligned}
\mathcal{C}(\mathbf{w}, \boldsymbol{\zeta})+\mathcal{B}(\boldsymbol{\zeta}, \mathbf{q}) & =\left(\mathbf{y}-\mathbf{y}_{\Omega}, \boldsymbol{\zeta}\right)_{\boldsymbol{L}^{2}(\Omega)} & & \forall \boldsymbol{\zeta} \in \mathbf{V}, \\
\mathcal{B}(\mathbf{w}, \psi) & =0 & & \forall \psi \in Q .
\end{aligned}\right.
$$

With this adjoint state at hand, the variational inequality (3.4) can be rewritten as

$$
(\overline{\mathbf{w}}+\vartheta \overline{\mathbf{u}}, \mathbf{u}-\overline{\mathbf{u}})_{L^{2}(\Omega)} \geq 0 \quad \forall \mathbf{u} \in \mathbf{U}_{a d} .
$$

We have thus arrived at the following optimality system: $(\overline{\mathbf{y}}, \overline{\mathrm{p}}, \overline{\mathbf{u}}) \in \mathbf{V} \times Q \times \mathbf{U}_{a d}$ is optimal for the PDE-constrained optimization problem (1.2)-(1.4) if and only if $(\overline{\mathbf{y}}, \overline{\mathbf{p}}, \overline{\mathbf{w}}, \overline{\mathbf{q}}, \overline{\mathbf{u}}) \in \mathbf{V} \times Q \times \mathbf{V} \times Q \times \mathbf{U}_{a d}$ solves

$$
\left\{\begin{aligned}
\mathcal{A}(\overline{\mathbf{y}}, \boldsymbol{\xi})-\mathcal{B}(\boldsymbol{\xi}, \overline{\mathbf{p}}) & =(\mathbf{f}+\overline{\mathbf{u}}, \boldsymbol{\xi})_{L^{2}(\Omega)}, & & \forall \boldsymbol{\xi} \in \mathbf{V}, \\
\mathcal{B}(\overline{\mathbf{y}}, \phi) & =0, & & \forall \phi \in Q, \\
\mathcal{C}(\overline{\mathbf{w}}, \boldsymbol{\zeta})+\mathcal{B}(\boldsymbol{\zeta}, \overline{\mathbf{q}}) & =\left(\overline{\mathbf{y}}-\mathbf{y}_{\Omega}, \boldsymbol{\zeta}\right)_{L^{2}(\Omega)}, & & \forall \boldsymbol{\zeta} \in \mathbf{V}, \\
\mathcal{B}(\overline{\mathbf{w}}, \psi) & =0, & & \forall \psi \in Q, \\
(\overline{\mathbf{w}}+\vartheta \overline{\mathbf{u}}, \mathbf{u}-\overline{\mathbf{u}})_{\boldsymbol{L}^{2}(\Omega)} & \geq 0, & &
\end{aligned}\right.
$$

see also [39, Section 2] and [34, Section 2] for similar results when the state equations (1.3) are the Stokes equations.

We finally recall the projection formula for the optimal control variable: the variational inequality in (3.6) can be equivalently written as [44, Chapter 2]

$$
\overline{\mathbf{u}}=\Pi_{[\mathbf{a}, \mathbf{b}]}\left(-\frac{1}{\vartheta} \overline{\mathbf{w}}\right) \quad \text { a.e. in } \Omega,
$$

where $\Pi_{[\mathbf{a}, \mathbf{b}]}(\boldsymbol{\zeta})(\boldsymbol{x}):=\min \{\mathbf{b}, \max \{\mathbf{a}, \boldsymbol{\zeta}(\boldsymbol{x})\}\}$ and it is understood componentwise. We note that

$$
\left\|\Pi_{[\mathbf{a}, \mathbf{b}]}(\boldsymbol{\xi})-\Pi_{[\mathbf{a}, \mathbf{b}]}(\boldsymbol{\zeta})\right\|_{\boldsymbol{L}^{2}(K)} \leq\|\boldsymbol{\xi}-\boldsymbol{\zeta}\|_{\boldsymbol{L}^{2}(K)} \quad \forall \boldsymbol{\xi}, \boldsymbol{\zeta} \in \mathbf{V} .
$$

4. Finite element discretization. We follow the optimize-then-discretize approach and introduce a numerical scheme to approximate the solution to (3.7). The scheme allows for the incorporation of stabilization terms into the standard Galerkin discretizations of the state and adjoint equations; no a priori relation between the stabilized terms is required. We refer the reader to Remark 4.1 below for a discussion regarding the advantages of the proposed approach when solving (1.2)-(1.4).

The stabilized scheme reads as follows: Find $\left(\overline{\mathbf{y}}_{\mathscr{T}}, \overline{\mathbf{p}}_{\mathscr{T}}, \overline{\mathbf{w}}_{\mathscr{T}}, \overline{\mathbf{q}}_{\mathscr{T}}, \overline{\mathbf{u}}_{\mathscr{T}}\right) \in \mathbf{V}(\mathscr{T}) \times$ $Q(\mathscr{T}) \times \mathbf{V}(\mathscr{T}) \times Q(\mathscr{T}) \times \mathbf{U}_{a d}(\mathscr{T})$ such that

$$
\left\{\begin{aligned}
\mathcal{A}\left(\overline{\mathbf{y}}_{\mathscr{T}}, \boldsymbol{\xi}\right)-\mathcal{B}\left(\boldsymbol{\xi}, \overline{\mathbf{p}}_{\mathscr{T}}\right)+\mathcal{S}\left(\overline{\mathbf{y}}_{\mathscr{T}}, \overline{\mathbf{p}}_{\mathscr{T}}, \mathbf{f}+\overline{\mathbf{u}}_{\mathscr{T}} ; \boldsymbol{\xi}\right) & =\left(\mathbf{f}+\overline{\mathbf{u}}_{\mathscr{T}}, \boldsymbol{\xi}\right)_{L^{2}(\Omega)} \\
\mathcal{B}\left(\overline{\mathbf{y}}_{\mathscr{T}}, \phi\right)+\mathcal{H}\left(\overline{\mathbf{y}}_{\mathscr{T}}, \overline{\mathbf{p}} \mathscr{T}, \mathbf{f}+\overline{\mathbf{u}}_{\mathscr{T}} ; \phi\right. & =0 \\
\mathcal{C}\left(\overline{\mathbf{w}}_{\mathscr{T}}, \boldsymbol{\zeta}\right)+\mathcal{B}\left(\boldsymbol{\zeta}, \overline{\mathbf{q}}_{\mathscr{T}}\right)+\mathcal{Q}\left(\overline{\mathbf{w}}_{\mathscr{T}}, \overline{\mathrm{q}}_{\mathscr{T}}, \overline{\mathbf{y}}_{\mathscr{T}}-\mathbf{y}_{\Omega} ; \boldsymbol{\zeta}\right) & =\left(\overline{\mathbf{y}}_{\mathscr{T}}-\mathbf{y}_{\Omega}, \boldsymbol{\zeta}\right)_{L^{2}(\Omega)} \\
\mathcal{B}\left(\overline{\mathbf{w}}_{\mathscr{T}}, \psi\right)+\mathcal{K}\left(\overline{\mathbf{w}}_{\mathscr{T}}, \overline{\mathbf{q}}_{\mathscr{T}}, \overline{\mathbf{y}}_{\mathscr{T}}-\mathbf{y}_{\Omega} ; \psi\right) & =0 \\
\left(\overline{\mathbf{w}}_{\mathscr{T}}+\vartheta \overline{\mathbf{u}}_{\mathscr{T}}, \mathbf{u}-\overline{\mathbf{u}}_{\mathscr{T}}\right)_{L^{2}(\Omega)} & \geq 0
\end{aligned}\right.
$$


for all $(\boldsymbol{\xi}, \phi, \boldsymbol{\zeta}, \psi, \mathbf{u}) \in \mathbf{V}(\mathscr{T}) \times Q(\mathscr{T}) \times \mathbf{V}(\mathscr{T}) \times Q(\mathscr{T}) \times \mathbf{U}_{a d}(\mathscr{T})$; the bilinear forms $\mathcal{A}, \mathcal{B}$ and $\mathcal{C}$ being defined as in (2.9). We consider the setting where the discrete spaces $\mathbf{V}(\mathscr{T})$ and $Q(\mathscr{T})$ are subspaces of $\mathbf{V}$ and $Q$, respectively, and the discrete set $\mathbf{U}_{a d}(\mathscr{T})$ is a subset of $\mathbf{U}_{a d}$. Hence, $\mathbf{V}(\mathscr{T}) \subset \mathbf{V}, Q(\mathscr{T}) \subset Q$ and $\mathbf{U}_{a d}(\mathscr{T}) \subset \mathbf{U}_{a d}$. The terms $\mathcal{S}$ and $\mathcal{H}$, and $\mathcal{Q}$ and $\mathcal{K}$ in (4.1), correspond to stabilization terms for the state and adjoint equations, respectively. Finally, we assume that $\mathbf{V}(\mathscr{T}), Q(\mathscr{T}), \mathbf{U}_{a d}(\mathscr{T}), \mathcal{S}$, $\mathcal{H}, \mathcal{Q}$ and $\mathcal{K}$ are such that at least one solution to (4.1) exists.

Remark 4.1 (optimize-then-discretize approach). In general, there are two approaches to approximate the solution to an optimal control problem: the optimizethen-discretize approach, that discretizes the associated optimality system, and the discretize-then-optimize approach, that first discretizes the continuous problem and then optimizes the obtained finite dimensional problem. We must immediately comment that these techniques do not always coincide [16, 19, 26, 29]. For a detailed discussion on these approaches and their respective advantages and disadvantages, we refer the reader to [26, Section 3.2] and [15, Chapter 3]. In [19], it was observed that, when solving an optimal control problem for a convection-reaction-difussion equation on the basis of the SUPG method, both approaches lead to substantially different results. Later, in [25], the authors continue with the study started in [19] and show that the failure to resolve boundary layers exhibited by the solution can pollute the numerical solution in the entire domain. In order to develop our a posteriori error analysis, we follow the optimize-then-discretize approach. This allows for the simple formulation (4.1) of the discrete optimality system and the incorporation of stabilization terms into the discrete state and adjoint equations; no a priori relationship between such stabilization terms is required. We remark that the latter property is particularly convenient since it allows for the use of the a posteriori error estimators that are already available in the literature. In contrast, the use of the discretize-thenoptimize approach imposes a relationship between the stabilization terms which could result in the presence of undesirable stabilization terms in the discrete formulation. If both terms $\mathcal{S}$ and $\mathcal{H}$ are symmetric and $\mathcal{Q}=\mathcal{S}$ and $\mathcal{K}=\mathcal{H}$, then the aforementioned approaches coincide; we refer the reader to [16] for details.

Before proceeding with the analysis of our method, it is instructive to comment on those advocated in the literature. Regarding the a priori theory, in the absence of control constraints, the design and analysis of numerical techniques for solving (1.2)(1.3), with $\mathbf{c}=\mathbf{0}$ and $\kappa=0$, have been investigated in several papers; see [14, 40, 42] and references therein. To the best of our knowledge, and again, for $\mathbf{c}=\mathbf{0}$ and $\kappa=0$, the first work that incorporates control constraints and analyzes stabilized schemes for (1.2)-(1.4) is [39]; the optimal control is discretized by using piecewise constant functions. The authors, on the basis of postprocessing techniques, provide a quadratic error estimate for the approximation of the optimal control variable [39, Theorem 2.8]. Subsequently, the authors of [34] extend the results of [39] and analyze nonconforming schemes for the discretization of the state and adjoint equations; in contrast to [39], the vector field is not assumed to be in $\mathbf{H}^{2}(\Omega) \cap \mathbf{W}^{1, \infty}(\Omega)$. In addition, [34] analyzes an anisotropic scheme for approximating the solution to (1.2)-(1.4) when $\Omega$ is not convex; a domain with a reentrant edge $(d=3)$ is considered. We conclude this paragraph by mentioning the reference [22], where the authors investigate numerical techniques for solving a modification of problem (1.2)-(1.4) that, in addition, includes constraints on the state variable.

Regarding the a posteriori error analysis, to the best of our knowledge, the first work to propose an error estimator for (1.2)-(1.4), with $\mathbf{c}=\mathbf{0}$ and $\kappa=0$, is [32]. In this 
work, the authors follow the discretize-then-optimize approach and obtain a discrete optimality system with no stabilization terms [32, equation (2.9)]. They propose an error estimator in a two-dimensional setting and analyze its reliability properties [32, Theorem 3.1]. However, there is no efficiency analysis. Later, an asymptotically exact ZZ-type a posteriori error estimator was proposed in [31]. The authors derive upper and lower bounds for the error in terms of the proposed estimator [31, Theorem 5.1] that relies on an error non-degeneracy condition [31, inequality (2.24)] and strong regularity assumptions on $(\overline{\mathbf{y}}, \overline{\mathrm{p}})$ : it is assumed to belong to $\mathbf{H}^{3}(\Omega) \cap \mathbf{V} \times \mathbf{H}^{1}(\Omega) \cap Q$ [31, Lemma 4.2]. In [20], the authors propose an a posteriori error estimator for (1.2)(1.4) but with the state equations (1.3) replaced by a Stokes-Darcy system: they study the reliability and efficiency properties of the proposed estimator. We also mention [35], where a similar PDE-constrained optimization problem has been analyzed but with the control constraint (1.4) replaced by the state constraint $\|\mathbf{y}\|_{\mathbf{L}^{2}(\Omega)} \leq \gamma$, where $\gamma>0$ : an error estimator is proposed and its reliability and efficiency properties are investigated. All the aforementioned references consider plain Galerkin discretizations for the state and adjoint equations, i.e., no stabilization terms are considered. We conclude this paragraph by mentioning the so-called dual weighted residual method (DWR) [13] and its applications to the optimal control of flow problems [11, 12].

Recently, the authors of [28] propose and analyze an a posteriori error estimator for problem (1.2)-(1.4) when $\kappa=0$ [28, Section 5]. The associated discrete optimal system incorporates stabilized terms, into the state and adjoint equations, that are based on the streamline-diffusion finite element method (SDFEM). On the basis of proposed and analyzed a posteriori error estimators for the state and adjoint equations, the authors derive an estimator for (1.2)-(1.4). We comment that the obtained upper bound for the error, in terms of the a posteriori error estimator, is not computable.

In this work we analyze a family of a posteriori error estimators in a unifying framework that incorporates a wide variety of standard and stabilized finite element methods.

5. A posteriori error analysis. In this section we derive and analyze a posteriori error estimators for the solution to the discretization (4.1) of the optimal control problem (3.7).

5.1. Reliability analysis. We begin this section by introducing the following notation. Let $\mathbf{e}_{\mathbf{y}}:=\overline{\mathbf{y}}-\overline{\mathbf{y}}_{\mathscr{T}}, \mathrm{e}_{\mathrm{p}}:=\overline{\mathrm{p}}-\overline{\mathrm{p}}_{\mathscr{T}}, \mathbf{e}_{\mathbf{w}}:=\overline{\mathbf{w}}-\overline{\mathbf{w}}_{\mathscr{T}}, \mathrm{e}_{\mathrm{q}}:=\overline{\mathrm{q}}-\overline{\mathrm{q}}_{\mathscr{T}}$ and $\mathbf{e}_{\mathbf{u}}:=\overline{\mathbf{u}}-\overline{\mathbf{u}}_{\mathscr{T}}$, where $(\overline{\mathbf{y}}, \overline{\mathbf{p}}, \overline{\mathbf{w}}, \overline{\mathbf{q}}, \overline{\mathbf{u}}) \in \mathbf{V} \times Q \times \mathbf{V} \times Q \times \mathbf{U}_{a d}$ is the solution to the optimality system (3.7) and $\left(\overline{\mathbf{y}}_{\mathscr{T}}, \overline{\mathbf{p}}_{\mathscr{T}}, \overline{\mathbf{w}}_{\mathscr{T}}, \overline{\mathbf{q}}_{\mathscr{T}}, \overline{\mathbf{u}}_{\mathscr{T}}\right) \in \mathbf{V}(\mathscr{T}) \times Q(\mathscr{T}) \times \mathbf{V}(\mathscr{T}) \times Q(\mathscr{T}) \times \mathbf{U}_{\text {ad }}(\mathscr{T})$ is its numerical approximation given as the solution to (4.1). The goal of this section is to obtain an upper bound for

$$
\left\|\left(\mathbf{e}_{\mathbf{y}}, \mathrm{e}_{\mathrm{p}}, \mathbf{e}_{\mathbf{w}}, \mathrm{e}_{\mathbf{q}}, \mathbf{e}_{\mathbf{u}}\right)\right\|_{\Omega}^{2}:=\sum_{K \in \mathscr{T}}\left\|\left(\mathbf{e}_{\mathbf{y}}, \mathrm{e}_{\mathrm{p}}, \mathbf{e}_{\mathbf{w}}, \mathrm{e}_{\mathbf{q}}, \mathbf{e}_{\mathbf{u}}\right)\right\|_{K}^{2}
$$

where

$$
\left\|\left(\mathbf{e}_{\mathbf{y}}, \mathrm{e}_{\mathrm{p}}, \mathbf{e}_{\mathbf{w}}, \mathrm{e}_{\mathbf{q}}, \mathbf{e}_{\mathbf{u}}\right)\right\|_{K}^{2}:=\left\|\mathbf{e}_{\mathbf{y}}\right\|_{\mathbf{V}, K}^{2}+\varrho\left\|\mathrm{e}_{\mathrm{p}}\right\|_{Q, K}^{2}+\left\|\mathbf{e}_{\mathbf{w}}\right\|_{\mathbf{V}, K}^{2}+\varrho\left\|\mathrm{e}_{\mathbf{q}}\right\|_{Q, K}^{2}+\left\|\mathbf{e}_{\mathbf{u}}\right\|_{\boldsymbol{L}^{2}(K)}^{2} .
$$

The norms $\|\cdot\|_{\mathbf{V}, K}$ and $\|\cdot\|_{Q, K}$ are defined as in (2.2) and the parameter $\varrho$ is a nonnegative constant that will be arbitrary in the analysis but fixed in the numerical experiments of Section 7 . 
The upper bound for the error (5.1) that we obtain is constructed using upper bounds on the error between the solution to the discretization (4.1) and auxiliary variables that we define in what follows. Let $(\hat{\mathbf{y}}, \hat{\mathbf{p}}) \in \mathbf{V} \times Q$ be the solution to

$$
\left\{\begin{aligned}
\mathcal{A}(\hat{\mathbf{y}}, \boldsymbol{\xi})-\mathcal{B}(\boldsymbol{\xi}, \hat{\mathbf{p}}) & =\left(\mathbf{f}+\overline{\mathbf{u}}_{\mathscr{T}}, \boldsymbol{\xi}\right)_{\boldsymbol{L}^{2}(\Omega)} & & \forall \boldsymbol{\xi} \in \mathbf{V}, \\
\mathcal{B}(\hat{\mathbf{y}}, \phi) & =0 & & \forall \phi \in Q .
\end{aligned}\right.
$$

We notice that, in view of (4.1), we have that $\left(\overline{\mathbf{y}}_{\mathscr{T}}, \overline{\mathbf{p}} \mathscr{T}\right) \in \mathbf{V}(\mathscr{T}) \times Q(\mathscr{T})$ satisfies

$$
\left\{\begin{aligned}
\mathcal{A}\left(\overline{\mathbf{y}}_{\mathscr{T}}, \boldsymbol{\xi}\right)-\mathcal{B}\left(\boldsymbol{\xi}, \overline{\mathbf{p}}_{\mathscr{T}}\right)+\mathcal{S}\left(\overline{\mathbf{y}}_{\mathscr{T}}, \overline{\mathbf{p}}_{\mathscr{T}}, \mathbf{f}+\overline{\mathbf{u}}_{\mathscr{T}} ; \boldsymbol{\xi}\right) & =\left(\mathbf{f}+\overline{\mathbf{u}}_{\mathscr{T}}, \boldsymbol{\xi}\right)_{L^{2}(\Omega)} \\
\mathcal{B}\left(\overline{\mathbf{y}}_{\mathscr{T}}, \phi\right)+\mathcal{H}\left(\overline{\mathbf{y}}_{\mathscr{T}}, \overline{\mathbf{p}}_{\mathscr{T}}, \mathbf{f}+\overline{\mathbf{u}}_{\mathscr{T}} ; \phi\right) & =0
\end{aligned}\right.
$$

for all $\boldsymbol{\xi} \in \mathbf{V}(\mathscr{T})$ and $\phi \in Q(\mathscr{T})$. Consequently, $\left(\overline{\mathbf{y}}_{\mathscr{T}}, \overline{\mathrm{p}}_{\mathscr{T}}\right)$ can be seen as a finite element approximation of the solution to (5.2). We thus make the following assumption:

Assumption 1. There exist quantities $\eta_{\mathbf{y}}$ and $\eta_{\mathrm{p}}$ which depend on the discrete solution and data and are such that

$$
\left\|\hat{\mathbf{y}}-\overline{\mathbf{y}}_{\mathscr{T}}\right\|_{\mathbf{V}, \Omega} \leq \eta_{\mathbf{y}} \text { and }\left\|\hat{\mathbf{p}}-\overline{\mathbf{p}}_{\mathscr{T}}\right\|_{Q, \Omega} \leq \eta_{\mathbf{p}}
$$

Let $(\hat{\mathbf{w}}, \hat{\mathbf{q}}) \in \mathbf{V} \times Q$ be the solution to

$$
\left\{\begin{aligned}
\mathcal{C}(\hat{\mathbf{w}}, \boldsymbol{\zeta})+\mathcal{B}(\boldsymbol{\zeta}, \hat{\mathbf{q}}) & =\left(\overline{\mathbf{y}}_{\mathscr{T}}-\mathbf{y}_{\Omega}, \boldsymbol{\zeta}\right)_{\boldsymbol{L}^{2}(\Omega)} & & \forall \boldsymbol{\zeta} \in \mathbf{V} \\
\mathcal{B}(\hat{\mathbf{w}}, \psi) & =0 & & \forall \psi \in Q .
\end{aligned}\right.
$$

We notice that, again in view of $(4.1),\left(\overline{\mathbf{w}}_{\mathscr{T}}, \overline{\mathbf{q}}_{\mathscr{T}}\right) \in \mathbf{V}(\mathscr{T}) \times Q(\mathscr{T})$ satisfies

$$
\left\{\begin{aligned}
\mathcal{C}\left(\overline{\mathbf{w}}_{\mathscr{T}}, \boldsymbol{\zeta}\right)+\mathcal{B}\left(\boldsymbol{\zeta}, \overline{\mathbf{q}}_{\mathscr{T}}\right)+\mathcal{Q}\left(\overline{\mathbf{w}}_{\mathscr{T}}, \overline{\mathbf{q}}_{\mathscr{T}}, \overline{\mathbf{y}}_{\mathscr{T}}-\mathbf{y}_{\Omega} ; \boldsymbol{\zeta}\right) & =\left(\overline{\mathbf{y}}_{\mathscr{T}}-\mathbf{y}_{\Omega}, \boldsymbol{\zeta}\right)_{L^{2}(\Omega)}, \\
\mathcal{B}\left(\overline{\mathbf{w}}_{\mathscr{T}}, \psi\right)+\mathcal{K}\left(\overline{\mathbf{w}}_{\mathscr{T}}, \overline{\mathbf{q}}_{\mathscr{T}}, \overline{\mathbf{y}}_{\mathscr{T}}-\mathbf{y}_{\Omega} ; \psi\right) & =0,
\end{aligned}\right.
$$

for all $\boldsymbol{\zeta} \in \mathbf{V}(\mathscr{T})$ and $\psi \in Q(\mathscr{T})$, and hence $\left(\overline{\mathbf{w}}_{\mathscr{T}}, \overline{\mathbf{q}}_{\mathscr{T}}\right)$ corresponds to a finite element approximation of the solution to (5.5). We thus make the following assumption:

Assumption 2. There exist quantities $\eta_{\mathbf{w}}$ and $\eta_{\mathbf{q}}$ which depend on the discrete solution and data and are such that

$$
\left\|\hat{\mathbf{w}}-\overline{\mathbf{w}}_{\mathscr{T}}\right\|_{\mathbf{V}, \Omega} \leq \eta_{\mathbf{w}} \text { and }\left\|\hat{\mathbf{q}}-\overline{\mathbf{q}}_{\mathscr{T}}\right\|_{Q, \Omega} \leq \eta_{\mathbf{q}} .
$$

We introduce the auxiliary control variable

$$
\tilde{\mathbf{u}}=\Pi_{[\mathbf{a}, \mathbf{b}]}\left(-\frac{1}{\vartheta} \overline{\mathbf{w}}_{\mathscr{T}}\right) \text {. }
$$

We define the error between this auxiliary control variable and $\overline{\mathbf{u}}_{\mathscr{T}}$ as follows:

$$
\eta_{\mathbf{u}}:=\left(\sum_{K \in \mathscr{T}} \eta_{\mathbf{u}, K}^{2}\right)^{1 / 2}, \text { with } \eta_{\mathbf{u}, K}:=\left\|\tilde{\mathbf{u}}-\overline{\mathbf{u}}_{\mathscr{T}}\right\|_{L^{2}(K)} .
$$

We also define

$$
\begin{gathered}
\mathfrak{C}_{\mathbf{y}}=2+2 \mu \mathrm{C}_{\Omega}^{6}+4(1+\varrho \omega)\left(\mathrm{C}_{\Omega}^{4}+\mu \mathrm{C}_{\Omega}^{8}+2 \mu \mathrm{C}_{\Omega}^{12}\right), \\
\mathfrak{C}_{\mathbf{w}}=2+\mu \mathrm{C}_{\Omega}^{2}+2 \mu(1+\varrho \omega)\left(\mathrm{C}_{\Omega}^{4}+2 \mathrm{C}_{\Omega}^{8}\right)
\end{gathered}
$$

and

$$
\mathfrak{C}_{\mathbf{u}}=2+2 \mu \mathrm{C}_{\Omega}^{8}+4(1+\varrho \omega)\left(\mathrm{C}_{\Omega}^{2}+2 \mathrm{C}_{\Omega}^{6}+\mu \mathrm{C}_{\Omega}^{10}+2 \mu \mathrm{C}_{\Omega}^{14}\right),
$$

with $\mu=4 \vartheta^{-2}$ and $\omega=\mathrm{C}_{\text {is }}^{2}\left(1+\mathrm{C}_{\mathrm{ct}}\right)^{2}$.

We now present the analysis through which we obtain an upper bound for the total error. 
Theorem 5.1 (global reliability). If Assumptions 1 and 2 hold, then

$$
\left\|\left(\mathbf{e}_{\mathbf{y}}, e_{\mathrm{p}}, \mathbf{e}_{\mathbf{w}}, \mathrm{e}_{\mathbf{q}}, \mathbf{e}_{\mathbf{u}}\right)\right\|_{\Omega}^{2} \leq \Upsilon^{2}
$$

where

$$
\Upsilon^{2}:=\mathfrak{C}_{\mathbf{y}} \eta_{\mathbf{y}}^{2}+2 \varrho \eta_{\mathrm{p}}^{2}+\mathfrak{C}_{\mathbf{w}} \eta_{\mathbf{w}}^{2}+2 \varrho \eta_{\mathrm{q}}^{2}+\mathfrak{C}_{\mathbf{u}} \eta_{\mathbf{u}}^{2}
$$

and $\mathfrak{C}_{\mathbf{y}}, \mathfrak{C}_{\mathbf{w}}$ and $\mathfrak{C}_{\mathbf{u}}$ are defined by (5.10), (5.11) and (5.12), respectively.

Proof. We proceed in 6 steps.

Step 1. The goal of this step is to control the term $\left\|\mathbf{e}_{\mathbf{u}}\right\|_{L^{2}(\Omega)}$. We begin with a simple application of the triangle inequality to write

$$
\left\|\mathbf{e}_{\mathbf{u}}\right\|_{L^{2}(\Omega)}^{2} \leq 2\|\overline{\mathbf{u}}-\tilde{\mathbf{u}}\|_{L^{2}(\Omega)}^{2}+2\left\|\tilde{\mathbf{u}}-\overline{\mathbf{u}}_{\mathscr{T}}\right\|_{L^{2}(\Omega)}^{2}=2\|\overline{\mathbf{u}}-\tilde{\mathbf{u}}\|_{L^{2}(\Omega)}^{2}+2 \eta_{\mathbf{u}}^{2},
$$

where $\tilde{\mathbf{u}}=\Pi_{[\mathbf{a}, \mathbf{b}]}\left(-\frac{1}{\vartheta} \overline{\mathbf{w}}_{\mathscr{T}}\right)$ and $\eta_{\mathbf{u}}$ is defined as in (5.9).

Let us now bound the first term on the right hand side of (5.15). To accomplish this task we first observe a key property that the auxiliary control variable $\tilde{\mathbf{u}}$ satisfies:

$$
\left(\overline{\mathbf{w}}_{\mathscr{T}}+\vartheta \tilde{\mathbf{u}}, \mathbf{u}-\tilde{\mathbf{u}}\right)_{\boldsymbol{L}^{2}(\Omega)} \geq 0 \quad \forall \mathbf{u} \in \mathbf{U}_{\mathrm{ad}}
$$

see Lemma 2.26 and Theorem 2.28 in [44]. Set $\mathbf{u}=\tilde{\mathbf{u}}$ in the variational inequality of (3.7) and $\mathbf{u}=\overline{\mathbf{u}}$ in (5.16). We thus obtain that

$$
(\overline{\mathbf{w}}+\vartheta \overline{\mathbf{u}}, \tilde{\mathbf{u}}-\overline{\mathbf{u}})_{L^{2}(\Omega)} \geq 0, \quad\left(\overline{\mathbf{w}}_{\mathscr{T}}+\vartheta \tilde{\mathbf{u}}, \overline{\mathbf{u}}-\tilde{\mathbf{u}}\right)_{L^{2}(\Omega)} \geq 0,
$$

and, consequently, that

$$
\vartheta\|\overline{\mathbf{u}}-\tilde{\mathbf{u}}\|_{L^{2}(\Omega)}^{2} \leq(\overline{\mathbf{w}}-\overline{\mathbf{w}} \mathscr{T}, \tilde{\mathbf{u}}-\overline{\mathbf{u}})_{L^{2}(\Omega)} .
$$

In order to bound the right hand side of (5.17), we first define $(\tilde{\mathbf{y}}, \tilde{\mathbf{p}}) \in \mathbf{V} \times Q$ as the solution to

$$
\left\{\begin{aligned}
\mathcal{A}(\tilde{\mathbf{y}}, \boldsymbol{\xi})-\mathcal{B}(\boldsymbol{\xi}, \tilde{\mathbf{p}}) & =(\mathbf{f}+\tilde{\mathbf{u}}, \boldsymbol{\xi})_{L^{2}(\Omega)} & & \forall \boldsymbol{\xi} \in \mathbf{V} \\
\mathcal{B}(\tilde{\mathbf{y}}, \phi) & =0 & & \forall \phi \in Q .
\end{aligned}\right.
$$

In addition, we define $(\tilde{\mathbf{w}}, \tilde{\mathbf{q}}) \in \mathbf{V} \times Q$ as the solution to

$$
\left\{\begin{aligned}
\mathcal{C}(\tilde{\mathbf{w}}, \boldsymbol{\zeta})+\mathcal{B}(\boldsymbol{\zeta}, \tilde{\mathbf{q}}) & =\left(\tilde{\mathbf{y}}-\mathbf{y}_{\Omega}, \boldsymbol{\zeta}\right)_{\boldsymbol{L}^{2}(\Omega)} & & \forall \boldsymbol{\zeta} \in \mathbf{V} \\
\mathcal{B}(\tilde{\mathbf{w}}, \psi) & =0 & & \forall \psi \in Q
\end{aligned}\right.
$$

Utilizing the states $\hat{\mathbf{w}}$ and $\tilde{\mathbf{w}}$ defined as the solutions to (5.5) and (5.19), respectively, we arrive at

$$
\begin{gathered}
\vartheta\|\overline{\mathbf{u}}-\tilde{\mathbf{u}}\|_{L^{2}(\Omega)}^{2} \leq(\overline{\mathbf{w}}-\tilde{\mathbf{w}}, \tilde{\mathbf{u}}-\overline{\mathbf{u}})_{L^{2}(\Omega)}+(\tilde{\mathbf{w}}-\hat{\mathbf{w}}, \tilde{\mathbf{u}}-\overline{\mathbf{u}})_{L^{2}(\Omega)}+(\hat{\mathbf{w}}-\overline{\mathbf{w}} \mathscr{T}, \tilde{\mathbf{u}}-\overline{\mathbf{u}})_{L^{2}(\Omega)} \\
\quad \leq(\overline{\mathbf{w}}-\tilde{\mathbf{w}}, \tilde{\mathbf{u}}-\overline{\mathbf{u}})_{L^{2}(\Omega)}+\frac{1}{\vartheta}\|\tilde{\mathbf{w}}-\hat{\mathbf{w}}\|_{L^{2}(\Omega)}^{2}+\frac{1}{\vartheta}\left\|\hat{\mathbf{w}}-\overline{\mathbf{w}}_{\mathscr{T}}\right\|_{L^{2}(\Omega)}^{2}+\frac{\vartheta}{2}\|\overline{\mathbf{u}}-\tilde{\mathbf{u}}\|_{L^{2}(\Omega)}^{2}
\end{gathered}
$$

upon using Cauchy-Schwarz and Young's inequalities. Hence,

$$
\|\overline{\mathbf{u}}-\tilde{\mathbf{u}}\|_{\boldsymbol{L}^{2}(\Omega)}^{2} \leq \frac{2}{\vartheta}(\overline{\mathbf{w}}-\tilde{\mathbf{w}}, \tilde{\mathbf{u}}-\overline{\mathbf{u}})_{\boldsymbol{L}^{2}(\Omega)}+\frac{2}{\vartheta^{2}}\left(\|\tilde{\mathbf{w}}-\hat{\mathbf{w}}\|_{\boldsymbol{L}^{2}(\Omega)}^{2}+\left\|\hat{\mathbf{w}}-\overline{\mathbf{w}}_{\mathscr{T}}\right\|_{\boldsymbol{L}^{2}(\Omega)}^{2}\right) .
$$

We proceed to bound $(\overline{\mathbf{w}}-\tilde{\mathbf{w}}, \tilde{\mathbf{u}}-\overline{\mathbf{u}})_{\boldsymbol{L}^{2}(\Omega)}$. To accomplish this task, we first notice that, since $(\overline{\mathbf{w}}, \overline{\mathbf{q}})$ solves the adjoint problem of the optimality system (3.7) and $(\tilde{\mathbf{w}}, \tilde{\mathbf{q}})$ 
solves (5.19), the fact that $\overline{\mathrm{p}}-\tilde{\mathrm{p}} \in Q$ implies that $\mathcal{B}(\overline{\mathbf{w}}-\tilde{\mathbf{w}}, \tilde{\mathrm{p}}-\overline{\mathrm{p}})=0$. Thus, since $(\overline{\mathbf{y}}, \overline{\mathbf{p}})$ and $(\tilde{\mathbf{y}}, \tilde{\mathbf{p}})$ solve $(3.7)$ and (5.18), respectively, we arrive at

$$
(\overline{\mathbf{u}}-\tilde{\mathbf{u}}, \overline{\mathbf{w}}-\tilde{\mathbf{w}})_{L^{2}(\Omega)}=\mathcal{A}(\overline{\mathbf{y}}-\tilde{\mathbf{y}}, \overline{\mathbf{w}}-\tilde{\mathbf{w}}) .
$$

We now invoke (2.10) and, again, the fact that $(\overline{\mathbf{w}}, \overline{\mathbf{q}})$ and $(\tilde{\mathbf{w}}, \tilde{\mathbf{q}})$ solve (3.7) and (5.19), respectively, to obtain that

$$
(\tilde{\mathbf{u}}-\overline{\mathbf{u}}, \overline{\mathbf{w}}-\tilde{\mathbf{w}})_{\boldsymbol{L}^{2}(\Omega)}=\mathcal{A}(\tilde{\mathbf{y}}-\overline{\mathbf{y}}, \overline{\mathbf{w}}-\tilde{\mathbf{w}})=\mathcal{C}(\overline{\mathbf{w}}-\tilde{\mathbf{w}}, \tilde{\mathbf{y}}-\overline{\mathbf{y}})=-\|\overline{\mathbf{y}}-\tilde{\mathbf{y}}\|_{L^{2}(\Omega)}^{2} \leq 0,
$$

upon noticing that, since $(\overline{\mathbf{y}}, \overline{\mathbf{p}})$ solves the state equations of the optimality system (3.7) and $(\tilde{\mathbf{y}}, \tilde{\mathbf{p}})$ solves $(5.18)$, the fact that $\overline{\mathbf{q}}-\tilde{\mathrm{q}} \in Q$ implies that $\mathcal{B}(\overline{\mathbf{y}}-\tilde{\mathbf{y}}, \overline{\mathbf{q}}-\tilde{\mathbf{q}})=0$.

Using the previous estimate in (5.20) we obtain that

$$
\|\overline{\mathbf{u}}-\tilde{\mathbf{u}}\|_{L^{2}(\Omega)}^{2} \leq \frac{2}{\vartheta^{2}}\|\tilde{\mathbf{w}}-\hat{\mathbf{w}}\|_{L^{2}(\Omega)}^{2}+\frac{2}{\vartheta^{2}}\left\|\hat{\mathbf{w}}-\overline{\mathbf{w}}_{\mathscr{T}}\right\|_{L^{2}(\Omega)}^{2} .
$$

The control of the second term on the right hand side of (5.22) follows from (2.6) and Assumption 2:

$$
\left\|\hat{\mathbf{w}}-\overline{\mathbf{w}}_{\mathscr{T}}\right\|_{\boldsymbol{L}^{2}(\Omega)}^{2} \leq \mathrm{C}_{\Omega}^{2} \eta_{\mathbf{w}}^{2} .
$$

We now turn our attention to bounding the term $\|\tilde{\mathbf{w}}-\hat{\mathbf{w}}\|_{L^{2}(\Omega)}$. Applying similar arguments to the ones that lead to (5.21) we obtain that

$$
\begin{aligned}
\|\tilde{\mathbf{w}}-\hat{\mathbf{w}}\|_{\mathbf{V}, \Omega}^{2} & =\mathcal{C}(\tilde{\mathbf{w}}-\hat{\mathbf{w}}, \tilde{\mathbf{w}}-\hat{\mathbf{w}})=\left(\tilde{\mathbf{y}}-\overline{\mathbf{y}}_{\mathscr{T}}, \tilde{\mathbf{w}}-\hat{\mathbf{w}}\right)_{L^{2}(\Omega)} \\
& \leq \mathrm{C}_{\Omega}\left\|\tilde{\mathbf{y}}-\overline{\mathbf{y}}_{\mathscr{T}}\right\|_{L^{2}(\Omega)}\|\tilde{\mathbf{w}}-\hat{\mathbf{w}}\|_{\mathbf{V}, \Omega},
\end{aligned}
$$

where we have also used (2.6). Consequently, $\|\tilde{\mathbf{w}}-\hat{\mathbf{w}}\|_{\boldsymbol{L}^{2}(\Omega)}^{2} \leq \mathrm{C}_{\Omega}^{4}\left\|\tilde{\mathbf{y}}-\overline{\mathbf{y}}_{\mathscr{T}}\right\|_{\boldsymbol{L}^{2}(\Omega)}^{2}$, upon using, again, (2.6). It thus suffices to bound $\left\|\tilde{\mathbf{y}}-\overline{\mathbf{y}}_{\mathscr{T}}\right\|_{\boldsymbol{L}^{2}(\Omega)}$. We proceed as follows:

$$
\left\|\tilde{\mathbf{y}}-\overline{\mathbf{y}}_{\mathscr{T}}\right\|_{L^{2}(\Omega)}^{2} \leq 2\|\tilde{\mathbf{y}}-\hat{\mathbf{y}}\|_{L^{2}(\Omega)}^{2}+2\left\|\hat{\mathbf{y}}-\overline{\mathbf{y}}_{\mathscr{T}}\right\|_{L^{2}(\Omega)}^{2} .
$$

To control the second term on the right hand side of the previous expression, we invoke Assumption 1 and (2.6). We thus conclude that

$$
\left\|\hat{\mathbf{y}}-\overline{\mathbf{y}}_{\mathscr{T}}\right\|_{\boldsymbol{L}^{2}(\Omega)}^{2} \leq \mathrm{C}_{\Omega}^{2} \eta_{\mathbf{y}}^{2} .
$$

To bound the first term, we employ that $(\hat{\mathbf{y}}, \hat{\mathbf{p}})$ and $(\tilde{\mathbf{y}}, \tilde{\mathbf{p}})$ solve $(5.2)$ and (5.18), respectively. This, on the basis of $\nabla \cdot \mathbf{c}=0$ and (2.6), yields

$$
\begin{aligned}
\|\tilde{\mathbf{y}}-\hat{\mathbf{y}}\|_{\mathbf{V}, \Omega}^{2} & =\mathcal{A}(\tilde{\mathbf{y}}-\hat{\mathbf{y}}, \tilde{\mathbf{y}}-\hat{\mathbf{y}})=\left(\tilde{\mathbf{u}}-\overline{\mathbf{u}}_{\mathscr{T}}, \tilde{\mathbf{y}}-\hat{\mathbf{y}}\right)_{L^{2}(\Omega)} \\
& \leq \mathrm{C}_{\Omega}\left\|\tilde{\mathbf{u}}-\overline{\mathbf{u}}_{\mathscr{T}}\right\|_{L^{2}(\Omega)}\|\tilde{\mathbf{y}}-\hat{\mathbf{y}}\|_{\mathbf{V}, \Omega},
\end{aligned}
$$

which allows us to conclude, in view of (5.9) and (2.6), that

$$
\|\tilde{\mathbf{y}}-\hat{\mathbf{y}}\|_{L^{2}(\Omega)}^{2} \leq \mathrm{C}_{\Omega}^{4} \eta_{\mathbf{u}}^{2}
$$

On the basis of (5.15) and (5.22), we combine our previous findings and arrive at

$$
\left\|\mathbf{e}_{\mathbf{u}}\right\|_{\mathbf{L}^{2}(\Omega)}^{2} \leq 2 \mu \mathrm{C}_{\Omega}^{6} \eta_{\mathbf{y}}^{2}+\mu \mathrm{C}_{\Omega}^{2} \eta_{\mathbf{w}}^{2}+\left(2+2 \mu \mathrm{C}_{\Omega}^{8}\right) \eta_{\mathbf{u}}^{2},
$$


where $\mu=4 \vartheta^{-2}$.

Step 2. The goal of this step is to bound $\left\|\mathbf{e}_{\mathbf{y}}\right\|_{\mathbf{V}, \Omega}$. To accomplish this task, we apply the triangle inequality and invoke Assumption 1. In fact,

$$
\left\|\mathbf{e}_{\mathbf{y}}\right\|_{\mathbf{V}, \Omega}^{2} \leq 2\|\overline{\mathbf{y}}-\hat{\mathbf{y}}\|_{\mathbf{V}, \Omega}^{2}+2\left\|\hat{\mathbf{y}}-\overline{\mathbf{y}}_{\mathscr{T}}\right\|_{\mathbf{V}, \Omega}^{2} \leq 2\|\overline{\mathbf{y}}-\hat{\mathbf{y}}\|_{\mathbf{V}, \Omega}^{2}+2 \eta_{\mathbf{y}}^{2}
$$

To control the remaining term we employ similar ideas to the ones that lead to (5.24). These arguments reveal that

$$
\|\overline{\mathbf{y}}-\hat{\mathbf{y}}\|_{\mathbf{V}, \Omega}^{2} \leq \mathrm{C}_{\Omega}^{2}\left\|\overline{\mathbf{u}}-\overline{\mathbf{u}}_{\mathscr{T}}\right\|_{\boldsymbol{L}^{2}(\Omega)}^{2},
$$

which combined with (5.25) and (5.26), implies the error estimate

$$
\left\|\mathbf{e}_{\mathbf{y}}\right\|_{\mathbf{V}, \Omega}^{2} \leq 2\left(2 \mu \mathbf{C}_{\Omega}^{8}+1\right) \eta_{\mathbf{y}}^{2}+2 \mu \mathrm{C}_{\Omega}^{4} \eta_{\mathbf{w}}^{2}+2 \mathrm{C}_{\Omega}^{2}\left(2+2 \mu \mathrm{C}_{\Omega}^{8}\right) \eta_{\mathbf{u}}^{2}
$$

Step 3. We now bound the term $\left\|\mathbf{e}_{\mathbf{w}}\right\|_{\mathbf{V}, \Omega}$. To accomplish this task, we use, again, the triangle inequality and Assumption 2 to obtain that

$$
\left\|\mathbf{e}_{\mathbf{w}}\right\|_{\mathbf{V}, \Omega}^{2} \leq 2\|\overline{\mathbf{w}}-\hat{\mathbf{w}}\|_{\mathbf{V}, \Omega}^{2}+2 \eta_{\mathbf{w}}^{2} .
$$

To bound $\|\overline{\mathbf{w}}-\hat{\mathbf{w}}\|_{\mathbf{V}, \Omega}^{2}$ we invoke the optimality system (3.7) and (5.5). In fact, the arguments that allow us to obtain (5.23) immediately yield

$$
\begin{aligned}
\|\overline{\mathbf{w}}-\hat{\mathbf{w}}\|_{\mathbf{V}, \Omega}^{2} & =\mathcal{C}(\overline{\mathbf{w}}-\hat{\mathbf{w}}, \overline{\mathbf{w}}-\hat{\mathbf{w}})=\left(\overline{\mathbf{y}}-\overline{\mathbf{y}}_{\mathscr{T}}, \overline{\mathbf{w}}-\hat{\mathbf{w}}\right)_{L^{2}(\Omega)} \\
& \leq\left\|\overline{\mathbf{y}}-\overline{\mathbf{y}}_{\mathscr{T}}\right\|_{L^{2}(\Omega)}\|\overline{\mathbf{w}}-\hat{\mathbf{w}}\|_{L^{2}(\Omega)}
\end{aligned}
$$

upon using a Cauchy-Schwarz inequality. In view of (2.6), we conclude that

$$
\|\overline{\mathbf{w}}-\hat{\mathbf{w}}\|_{\mathbf{V}, \Omega}^{2} \leq \mathrm{C}_{\Omega}^{4}\left\|\overline{\mathbf{y}}-\overline{\mathbf{y}}_{\mathscr{T}}\right\|_{\mathbf{V}, \Omega}^{2},
$$

which, combined with the estimates (5.28) and (5.29), yields

$$
\left\|\mathbf{e}_{\mathbf{w}}\right\|_{\mathbf{V}, \Omega}^{2} \leq 4 \mathrm{C}_{\Omega}^{4}\left(2 \mu \mathrm{C}_{\Omega}^{8}+1\right) \eta_{\mathbf{y}}^{2}+2\left(2 \mu \mathrm{C}_{\Omega}^{8}+1\right) \eta_{\mathbf{w}}^{2}+4 \mathrm{C}_{\Omega}^{6}\left(2+2 \mu \mathrm{C}_{\Omega}^{8}\right) \eta_{\mathbf{u}}^{2} .
$$

Step 4. We now bound $\left\|\mathrm{e}_{\mathrm{p}}\right\|_{Q \Omega \Omega}$. We start with a simple application of the triangle inequality and Assumption 1:

$$
\left\|\mathrm{e}_{\mathrm{p}}\right\|_{Q, \Omega}^{2} \leq 2\|\overline{\mathrm{p}}-\hat{\mathrm{p}}\|_{Q, \Omega}^{2}+2\|\hat{\mathrm{p}}-\overline{\mathrm{p}} \mathscr{T}\|_{Q, \Omega}^{2} \leq 2\|\overline{\mathrm{p}}-\hat{\mathrm{p}}\|_{Q, \Omega}^{2}+2 \eta_{\mathrm{p}}^{2}
$$

we recall that $(\hat{\mathbf{y}}, \hat{\mathbf{p}})$ solves $(5.2)$. To control the first term on the right hand side of the previous expression, we utilize the inf-sup condition (2.15):

$$
\|\overline{\mathrm{p}}-\hat{\mathrm{p}}\|_{Q, \Omega} \leq \mathrm{C}_{\text {is }} \sup _{\boldsymbol{\xi} \in \mathbf{V} \backslash\{\mathbf{0}\}} \frac{\mathcal{B}(\boldsymbol{\xi}, \overline{\mathrm{p}}-\hat{\mathrm{p}})}{\|\boldsymbol{\xi}\|_{\mathbf{V}, \Omega}} .
$$

Since $(\overline{\mathbf{y}}, \overline{\mathbf{p}})$ and $(\hat{\mathbf{y}}, \hat{\mathbf{p}})$ solve (3.7) and (5.2), respectively, we conclude that

$$
\begin{aligned}
\mathcal{B}(\boldsymbol{\xi}, \overline{\mathrm{p}}-\hat{\mathbf{p}}) & =\mathcal{A}(\overline{\mathbf{y}}-\hat{\mathbf{y}}, \boldsymbol{\xi})-\left(\overline{\mathbf{u}}-\overline{\mathbf{u}}_{\mathscr{T}}, \boldsymbol{\xi}\right)_{\boldsymbol{L}^{2}(\Omega)} \\
& \leq\left(\mathrm{C}_{\mathrm{ct}}\|\overline{\mathbf{y}}-\hat{\mathbf{y}}\|_{\mathbf{V}, \Omega}+\mathrm{C}_{\Omega}\left\|\overline{\mathbf{u}}-\overline{\mathbf{u}}_{\mathscr{T}}\right\|_{\boldsymbol{L}^{2}(\Omega)}\right)\|\boldsymbol{\xi}\|_{\mathbf{V}, \Omega}
\end{aligned}
$$

upon using (2.6) and (2.12). In view of (5.27) we thus arrive at

$$
\mathcal{B}(\boldsymbol{\xi}, \overline{\mathrm{p}}-\hat{\mathrm{p}}) \leq \mathrm{C}_{\Omega}\left(1+\mathrm{C}_{\mathrm{ct}}\right)\left\|\overline{\mathbf{u}}-\overline{\mathbf{u}}_{\mathscr{T}}\right\|_{\boldsymbol{L}^{2}(\Omega)}\|\boldsymbol{\xi}\|_{\mathbf{V}, \Omega}
$$


This and (5.32) imply that $\|\overline{\mathrm{p}}-\hat{\mathrm{p}}\|_{Q, \Omega} \leq \mathrm{C}_{\text {is }} \mathrm{C}_{\Omega}\left(1+\mathrm{C}_{\mathrm{ct}}\right)\left\|\overline{\mathbf{u}}-\overline{\mathbf{u}}_{\mathscr{T}}\right\|_{\boldsymbol{L}^{2}(\Omega)}$. Thus,

$$
\left\|\mathrm{e}_{\mathrm{p}}\right\|_{Q, \Omega}^{2} \leq 2 \omega \mathrm{C}_{\Omega}^{2}\left\|\overline{\mathbf{u}}-\overline{\mathbf{u}}_{\mathscr{T}}\right\|_{L^{2}(\Omega)}^{2}+2 \eta_{\mathrm{p}}^{2},
$$

where $\omega=\mathrm{C}_{\text {is }}^{2}\left(1+\mathrm{C}_{\mathrm{ct}}\right)^{2}$. We conclude the estimate for $\left\|\mathrm{e}_{\mathrm{p}}\right\|_{Q, \Omega}^{2}$ by invoking (5.25):

$$
\left\|\mathrm{e}_{\mathrm{p}}\right\|_{Q, \Omega}^{2} \leq 4 \mu \omega \mathrm{C}_{\Omega}^{8} \eta_{\mathbf{y}}^{2}+2 \mu \omega \mathrm{C}_{\Omega}^{4} \eta_{\mathbf{w}}^{2}+2 \omega \mathrm{C}_{\Omega}^{2}\left(2+2 \mu \mathrm{C}_{\Omega}^{8}\right) \eta_{\mathbf{u}}^{2}+2 \eta_{\mathrm{p}}^{2}
$$

Step 5. We bound $\left\|\mathrm{e}_{\mathrm{q}}\right\|_{Q, \Omega}$. Similar arguments to the ones employed in the previous step yield

$$
\left\|\mathrm{e}_{\mathrm{q}}\right\|_{Q, \Omega}^{2} \leq 2\|\overline{\mathrm{q}}-\hat{\mathrm{q}}\|_{Q, \Omega}^{2}+2\|\hat{\mathrm{q}}-\overline{\mathrm{q}} \mathscr{T}\|_{Q, \Omega}^{2} \leq 2\|\overline{\mathrm{q}}-\hat{\mathrm{q}}\|_{Q, \Omega}^{2}+2 \eta_{\mathrm{q}}^{2}
$$

and

$$
\begin{aligned}
\|\overline{\mathrm{q}}-\hat{\mathrm{q}}\|_{Q, \Omega} & \leq \mathrm{C}_{\text {is }} \sup _{\boldsymbol{\zeta} \in \mathbf{V} \backslash\{\mathbf{0}\}} \frac{\left(\overline{\mathbf{y}}-\overline{\mathbf{y}}_{\mathscr{T}}, \boldsymbol{\zeta}\right)_{\boldsymbol{L}^{2}(\Omega)}-\mathcal{C}(\overline{\mathbf{w}}-\hat{\mathbf{w}}, \boldsymbol{\zeta})}{\|\boldsymbol{\zeta}\|_{\mathbf{V}, \Omega}} \\
& \leq \mathrm{C}_{\text {is }}\left(\mathrm{C}_{\Omega}^{2}\left\|\overline{\mathbf{y}}-\overline{\mathbf{y}}_{\mathscr{T}}\right\|_{\mathbf{V}, \Omega}+\mathrm{C}_{\mathrm{ct}}\|\overline{\mathbf{w}}-\hat{\mathbf{w}}\|_{\mathbf{V}, \Omega}\right) .
\end{aligned}
$$

We finally use (5.30), and conclude that $\|\overline{\mathrm{q}}-\hat{\mathrm{q}}\|_{Q, \Omega} \leq \mathrm{C}_{\text {is }} \mathrm{C}_{\Omega}^{2}\left(1+\mathrm{C}_{\mathrm{ct}}\right)\left\|\overline{\mathbf{y}}-\overline{\mathbf{y}}_{\mathscr{T}}\right\|_{\mathbf{V}, \Omega}$, and then that

$$
\left\|\mathrm{e}_{\mathrm{q}}\right\|_{Q, \Omega}^{2} \leq 2 \omega \mathrm{C}_{\Omega}^{4}\left\|\overline{\mathbf{y}}-\overline{\mathbf{y}}_{\mathscr{T}}\right\|_{\mathbf{V}, \Omega}^{2}+2 \eta_{\mathbf{q}}^{2}
$$

where, we recall that, $\omega=\mathrm{C}_{\mathrm{is}}^{2}\left(1+\mathrm{C}_{\mathrm{ct}}\right)^{2}$. Consequently,

$$
\left\|\mathrm{e}_{\mathbf{q}}\right\|_{Q, \Omega}^{2} \leq 4 \omega \mathrm{C}_{\Omega}^{4}\left(2 \mu \mathrm{C}_{\Omega}^{8}+1\right) \eta_{\mathbf{y}}^{2}+4 \mu \omega \mathrm{C}_{\Omega}^{8} \eta_{\mathbf{w}}^{2}+4 \omega \mathrm{C}_{\Omega}^{6}\left(2+2 \mu \mathrm{C}_{\Omega}^{8}\right) \eta_{\mathbf{u}}^{2}+2 \eta_{\mathbf{q}}^{2} .
$$

Step 6. Combining (5.25), (5.28), (5.31), (5.34) and (5.36) allows us to arrive at (5.13).

It is important in a posteriori error analysis to have an upper bound for the error that is in terms of local error indicators. Such a bound follows from Theorem 5.1 under the following two assumptions.

Assumption 3. There exist quantities $\eta_{\mathbf{y}, K}$ and $\eta_{\mathrm{p}, K}$ that depend on the discrete solution and data and are such that

$$
\left\|\hat{\mathbf{y}}-\overline{\mathbf{y}}_{\mathscr{T}}\right\|_{\mathbf{V}, \Omega}^{2} \leq \sum_{K \in \mathscr{T}} \eta_{\mathbf{y}, K}^{2} \text { and }\left\|\hat{\mathbf{p}}-\overline{\mathbf{p}}_{\mathscr{T}}\right\|_{Q, \Omega}^{2} \leq \sum_{K \in \mathscr{T}} \eta_{\mathbf{p}, K}^{2}
$$

Assumption 4. There exist quantities $\eta_{\mathbf{w}, K}$ and $\eta_{\mathbf{q}, K}$ that depend on the discrete solution and data and are such that

$$
\left\|\hat{\mathbf{w}}-\overline{\mathbf{w}}_{\mathscr{T}}\right\|_{\mathbf{V}, \Omega}^{2} \leq \sum_{K \in \mathscr{T}} \eta_{\mathbf{w}, K}^{2} \text { and }\|\hat{\mathbf{q}}-\overline{\mathbf{q}} \mathscr{T}\|_{Q, \Omega}^{2} \leq \sum_{K \in \mathscr{T}} \eta_{\mathbf{q}, K}^{2} .
$$

Theorem 5.2 (global reliability). If Assumptions 3 and 4 hold, then

$$
\left\|\left(\mathbf{e}_{\mathbf{y}}, \mathrm{e}_{\mathbf{p}}, \mathbf{e}_{\mathbf{w}}, \mathrm{e}_{\mathbf{q}}, \mathbf{e}_{\mathbf{u}}\right)\right\|_{\Omega}^{2} \leq \sum_{K \in \mathscr{T}} \Upsilon_{K}^{2}
$$

where

$$
\Upsilon_{K}^{2}:=\mathfrak{C}_{\mathbf{y}} \eta_{\mathbf{y}, K}^{2}+2 \varrho \eta_{\mathbf{p}, K}^{2}+\mathfrak{C}_{\mathbf{w}} \eta_{\mathbf{w}, K}^{2}+2 \varrho \eta_{\mathbf{q}, K}^{2}+\mathfrak{C}_{\mathbf{u}} \eta_{\mathbf{u}, K}^{2},
$$

and $\mathfrak{C}_{\mathbf{y}}, \mathfrak{C}_{\mathbf{w}}$ and $\mathfrak{C}_{\mathbf{u}}$ are defined by (5.10), (5.11) and (5.12), respectively. 
Proof. In view of Assumptions 3 and 4, the proof follows from a simple application of the result of Theorem 5.1.

Remark 5.3 (Assumptions 1-4). Assumptions 3 and 4 are at the heart of a posteriori error analysis $[3,36,46]$. They guarantee the existence of local quantities $\eta_{\mathbf{y}, K}, \eta_{\mathbf{p}, K}, \eta_{\mathbf{w}, K}$, and $\eta_{\mathbf{q}, K}$ that satisfy the estimates (5.37) and (5.38). This allows us to derive the a posteriori error estimate (5.39) which gives an upper bound for the error that is in terms of the local quantities $\Upsilon_{K}$. The quantities that are defined by (5.40) provide information beyond asymptotics and can be used to adaptively refine the underlying mesh. Although it may seems that, in view of Assumptions $\mathbf{3}$ and 4, the weaker Assumptions $\mathbf{1}$ and $\mathbf{2}$ are superfluous, there are a posteriori error estimators, such as the one developed in [1], which are such that the bounds in (5.4) and (5.7) are tighter than the ones in (5.37) and (5.38); see [1, Theorem 5.4]. Consequently, the upper bound in (5.13) that is in terms of $\Upsilon$, defined as in (5.14), is tighter than the upper bound that is in terms of $\Upsilon_{K}$. In such a setting, if the quantity $\Upsilon$ is computable, it would be preferable to use $\Upsilon$ as a stopping criterion in an adaptive algorithm.

Theorem 5.2 can be used to obtain guaranteed upper bounds on the error if the value of a $\beta$ satisfying (2.14) is known and the quantities $\eta_{\mathbf{y}, K}, \eta_{\mathrm{p}, K}, \eta_{\mathbf{w}, K}$ and $\eta_{\mathbf{q}, K}$ are computable. If this is not the case then Theorem 5.2 can still be used to arrive at an a posteriori error estimator under the following assumption.

Assumption 5. There exist computable quantities $\tilde{\eta}_{\mathbf{y}, K}, \tilde{\eta}_{\mathrm{p}, K}, \tilde{\eta}_{\mathbf{w}, K}$ and $\tilde{\eta}_{\mathbf{q}, K}$ which are such that $\eta_{\mathbf{y}, K} \lesssim \tilde{\eta}_{\mathbf{y}, K}, \eta_{\mathbf{p}, K} \lesssim \tilde{\eta}_{\mathbf{p}, K}, \eta_{\mathbf{w}, K} \lesssim \tilde{\eta}_{\mathbf{w}, K}$ and $\eta_{\mathbf{q}, K} \lesssim \tilde{\eta}_{\mathbf{q}, K}$ for all $K \in \mathscr{T}$.

Corollary 5.4 (global reliability). If Assumptions 3, 4 and 5 hold, then

$$
\left\|\left(\mathbf{e}_{\mathbf{y}}, \mathrm{e}_{\mathbf{p}}, \mathbf{e}_{\mathbf{w}}, \mathrm{e}_{\mathbf{q}}, \mathbf{e}_{\mathbf{u}}\right)\right\|_{\Omega}^{2} \lesssim \tilde{\Upsilon}^{2}:=\sum_{K \in \mathscr{T}} \tilde{\Upsilon}_{K}^{2}
$$

where

$$
\tilde{\Upsilon}_{K}^{2}:=\tilde{\eta}_{\mathbf{y}, K}^{2}+\tilde{\eta}_{\mathbf{p}, K}^{2}+\tilde{\eta}_{\mathbf{w}, K}^{2}+\tilde{\eta}_{\mathbf{q}, K}^{2}+\tilde{\eta}_{\mathbf{u}, K}^{2} .
$$

Proof. Upon invoking Assumptions 3, 4 and 5, the estimate (5.41) is a consequence of Theorem 5.2.

Remark 5.5 (Assumptions 5). The upper bounds that feature in the estimates of Assumptions $\mathbf{3}$ and $\mathbf{4}$ may not be computable. In fact, in the literature there are several a posteriori error estimates where the upper bound cannot be computed because of the presence of unknown constants; see, for instance, [2, 9, 43, 45, 47]. In order to include this type of a posteriori error estimate in our analysis, we consider Assumption 5, which guarantees that the upper bounds that feature in Assumptions $\mathbf{3}$ and $\mathbf{4}$ can be bounded by constants, whose value may not be known, multiplied by computable quantities. Note that these constants are equal to 1 if the upper bounds in Assumptions 3 and $\mathbf{4}$ are computable.

5.2. Efficiency analysis. In this section we prove the local efficiency of the a posteriori error indicators $\Upsilon_{K}$ and $\tilde{\Upsilon}_{K}$ defined by (5.40) and (5.42), respectively. In what follows we will assume that Assumptions 3, 4 and $\mathbf{5}$ are satisfied and that $\varrho \neq 0$. In addition, we make two further assumptions. To state them, we first define, for nonnegative integers $l$, the discrete space

$$
\mathbb{P}_{l}(\mathscr{T})=\left\{\mathbf{v} \in \mathbf{L}^{2}(\Omega): \mathbf{v}_{\mid K} \in \mathbb{P}_{l}(K)^{d} \text { for all } K \in \mathscr{T}\right\} .
$$


Our first additional assumption reads as follows:

Assumption 6. The spaces $\mathbf{V}(\mathscr{T})$ and $Q(\mathscr{T})$ and the set $\mathbf{U}_{a d}(\mathscr{T})$ are such that

- $\mathbf{V}(\mathscr{T})=\mathbf{V} \cap \mathbb{P}_{l_{\mathbf{V}}}(\mathscr{T})$ for some positive integer $l_{\mathbf{V}}$,

- $Q(\mathscr{T})=Q \cap \mathbb{P}_{l_{Q}}(\mathscr{T})$ for some nonnegative integer $l_{Q}$ or $Q(\mathscr{T})=Q \cap \mathbb{P}_{l_{Q}}(\mathscr{T}) \cap$ $H^{1}(\Omega)$ for some positive integer $l_{Q}$,

- $\mathbf{U}_{a d}(\mathscr{T})=\mathbf{U}_{a d} \cap \mathbb{P}_{l_{\mathbf{U}}}(\mathscr{T})$ for some nonnegative integer $l_{\mathbf{U}}$ or $\mathbf{U}_{a d}(\mathscr{T})=$ $\mathbf{U}_{a d} \cap \mathbb{P}_{l_{\mathbf{U}}}(\mathscr{T}) \cap H^{1}(\Omega)$ for some positive integer $l_{\mathbf{U}}$.

For $K \in \mathscr{T}$, we define the following residuals and oscillation terms:

$$
\mathcal{R}_{K}^{\mathrm{st}}:=\Pi_{K, m}(\mathbf{f})+\overline{\mathbf{u}}_{\mathscr{T} \mid K}+\varepsilon \Delta \overline{\mathbf{y}}_{\mathscr{T} \mid K}-\Pi_{K, m}\left((\mathbf{c} \cdot \nabla) \overline{\mathbf{y}}_{\mathscr{T} \mid K}\right)-\kappa \overline{\mathbf{y}}_{\mathscr{T} \mid K}-\nabla \overline{\mathbf{p}}_{\mathscr{T} \mid K},
$$

$$
\mathcal{R}_{K}^{\mathrm{ad}}:=\overline{\mathbf{y}}_{\mathscr{T} \mid K}-\Pi_{K, m}\left(\mathbf{y}_{\Omega}\right)+\varepsilon \Delta \overline{\mathbf{w}}_{\mathscr{T} \mid K}+\Pi_{K, m}\left((\mathbf{c} \cdot \nabla) \overline{\mathbf{w}}_{\mathscr{T} \mid K}\right)-\kappa \overline{\mathbf{w}}_{\mathscr{T} \mid K}+\nabla \overline{\mathbf{q}}_{\mathscr{T} \mid K}
$$

$$
\mathbf{o s c}_{K}^{\text {st }}:=\mathbf{f}-\Pi_{K, m}(\mathbf{f})-\left((\mathbf{c} \cdot \nabla) \overline{\mathbf{y}}_{\mathscr{T} \mid K}-\Pi_{K, m}\left((\mathbf{c} \cdot \nabla) \overline{\mathbf{y}}_{\mathscr{T} \mid K}\right)\right),
$$

and

$$
\mathbf{o s c}_{K}^{\mathrm{ad}}:=-\left(\mathbf{y}_{\Omega}-\Pi_{K, m}\left(\mathbf{y}_{\Omega}\right)\right)+\left((\mathbf{c} \cdot \nabla) \overline{\mathbf{w}}_{\mathscr{T} \mid K}-\Pi_{K, m}\left((\mathbf{c} \cdot \nabla) \overline{\mathbf{w}}_{\mathscr{T} \mid K}\right)\right),
$$

where $m=\max \left\{l_{\mathbf{V}}, l_{Q}-1, l_{\mathbf{U}}\right\}$. We recall that the operator $\Pi_{K, m}$ is defined as in (2.3), and notice that, in view of the choice of $m$, we have the following invariance property: $\Pi_{K, m}\left(\boldsymbol{\mathcal { R }}_{K}^{\text {st }}\right)=\mathcal{R}_{K}^{\text {st }}$ and $\Pi_{K, m}\left(\boldsymbol{\mathcal { R }}_{K}^{\text {ad }}\right)=\mathcal{R}_{K}^{\text {ad }}$. For $\gamma \in \mathcal{F}_{I}$, we define

$$
\llbracket \boldsymbol{\mathcal { R }}_{\gamma}^{\mathrm{st}} \rrbracket:=\sum_{K \in \Omega_{\gamma}} \boldsymbol{\mathcal { R }}_{\gamma, K}^{\mathrm{st}} \quad \text { with } \quad \boldsymbol{\mathcal { R }}_{\gamma, K}^{\mathrm{st}}:=-\varepsilon\left(\boldsymbol{n}_{\gamma}^{K} \cdot \nabla\right) \overline{\mathbf{y}}_{\mathscr{T} \mid K}+\overline{\mathbf{p}}_{\mathscr{T} \mid K} \boldsymbol{n}_{\gamma}^{K},
$$

and

$$
\llbracket \mathcal{R}_{\gamma}^{\mathrm{ad}} \rrbracket:=\sum_{K \in \Omega_{\gamma}} \boldsymbol{\mathcal { R }}_{\gamma, K}^{\mathrm{ad}} \quad \text { with } \quad \boldsymbol{\mathcal { R }}_{\gamma, K}^{\mathrm{ad}}:=-\varepsilon\left(\boldsymbol{n}_{\gamma}^{K} \cdot \nabla\right) \overline{\mathbf{w}}_{\mathscr{T} \mid K}-\overline{\mathrm{q}}_{\mathscr{T} \mid K} \boldsymbol{n}_{\gamma}^{K}
$$

We now state our final assumption.

Assumption 7. For all $K \in \mathscr{T}$, the computable quantities $\tilde{\eta}_{\mathbf{y}, K}, \tilde{\eta}_{\mathrm{p}, K}, \tilde{\eta}_{\mathbf{w}, K}$, and $\tilde{\eta}_{\mathrm{q}, K}$, introduced in Assumption 5, are such that

$$
\begin{aligned}
\tilde{\Upsilon}_{K}^{2} \lesssim & \left\|\nabla \cdot \overline{\mathbf{y}}_{\mathscr{T}}\right\|_{L^{2}(K)}^{2}+\left\|\nabla \cdot \overline{\mathbf{w}}_{\mathscr{T}}\right\|_{L^{2}(K)}^{2}+\sum_{K^{\prime} \in \hat{\mathscr{T}}_{K}} h_{K}^{2}\left(\left\|\mathcal{R}_{K^{\prime}}^{\mathrm{st}}\right\|_{L^{2}\left(K^{\prime}\right)}^{2}+\left\|\mathcal{R}_{K^{\prime}}^{\mathrm{ad}}\right\|_{L^{2}\left(K^{\prime}\right)}^{2}\right) \\
& +\sum_{\gamma \in \hat{\mathcal{F}}_{K}} h_{K}\left(\left\|\llbracket \mathcal{R}_{\gamma}^{\mathrm{st}}\right\|\left\|_{\boldsymbol{L}^{2}(\gamma)}^{2}+\right\| \llbracket \mathcal{R}_{\gamma}^{\mathrm{ad}} \rrbracket \|_{\boldsymbol{L}^{2}(\gamma)}^{2}\right) \\
& +\sum_{K^{\prime} \in \hat{\mathscr{T}}_{K}} h_{K}^{2}\left(\left\|\mathbf{o s c}_{K^{\prime}}^{\mathrm{st}}\right\|_{\boldsymbol{L}^{2}\left(K^{\prime}\right)}^{2}+\left\|\mathbf{o s c}_{K^{\prime}}^{\mathrm{ad}}\right\|_{\boldsymbol{L}^{2}\left(K^{\prime}\right)}^{2}\right)+\eta_{\mathbf{u}, K}^{2}
\end{aligned}
$$

where $\hat{\mathscr{T}}_{K} \subset \mathscr{T}$ and $\hat{\mathcal{F}}_{K} \subset \mathcal{F}_{I}$.

Under Assumptions 3, 4, 5, 6 and $\mathbf{7}$ we present an efficiency analysis. We start by noting that, since $\Upsilon_{K} \lesssim \tilde{\Upsilon}_{K}$, we only need to bound terms that appear on the right hand side of (5.50). 
We first invoke integration by parts and (3.1) to conclude that

$$
\begin{aligned}
& \sum_{K \in \mathscr{T}}\left(\mathcal{R}_{K}^{\mathrm{st}}, \boldsymbol{\xi}\right)_{\boldsymbol{L}^{2}(K)}+\sum_{\gamma \in \mathcal{F}_{I}}\left(\llbracket \mathcal{R}_{\gamma}^{\mathrm{st}} \rrbracket, \boldsymbol{\xi}\right)_{\boldsymbol{L}^{2}(\gamma)} \\
= & \mathcal{A}\left(\mathbf{e}_{\mathbf{y}}, \boldsymbol{\xi}\right)+\mathcal{B}\left(\boldsymbol{\xi}, \mathrm{e}_{\mathbf{p}}\right)-\left(\mathbf{e}_{\mathbf{u}}, \boldsymbol{\xi}\right)_{\boldsymbol{L}^{2}(\Omega)}-\sum_{K \in \mathscr{T}}\left(\mathbf{o s c}_{K}^{\mathrm{st}}, \boldsymbol{\xi}\right)_{\boldsymbol{L}^{2}(K)} \quad \forall \boldsymbol{\xi} \in \mathbf{V} .
\end{aligned}
$$

We now apply standard bubble function arguments $[3,46]$ to this equation to obtain

$$
\left\|\boldsymbol{R}_{K}^{\text {st }}\right\|_{L^{2}(K)}^{2} \lesssim h_{K}^{-2}\left(\left\|\mathbf{e}_{\mathbf{y}}\right\|_{\mathbf{V}, K}^{2}+\varrho\left\|\mathbf{e}_{\mathbf{p}}\right\|_{Q, K}^{2}\right)+\left\|\mathbf{e}_{\mathbf{u}}\right\|_{L^{2}(K)}^{2}+\left\|\mathbf{o s c}_{K}^{\text {st }}\right\|_{\boldsymbol{L}^{2}(K)}^{2}
$$

for $K \in \mathscr{T}$, and that, for $\gamma \in \mathcal{F}_{I}$,

$$
\begin{aligned}
\left\|\llbracket \mathcal{R}_{\gamma}^{\mathrm{st}} \rrbracket\right\|_{\boldsymbol{L}^{2}(\gamma)}^{2} \lesssim & \sum_{K^{\prime} \in \Omega_{\gamma}}\left(h_{K^{\prime}}^{-1}\left(\left\|\mathbf{e}_{\mathbf{y}}\right\|_{\mathbf{V}, K^{\prime}}^{2}+\varrho\left\|\mathrm{e}_{\mathrm{p}}\right\|_{Q, K^{\prime}}^{2}\right)\right. \\
& \left.+h_{K^{\prime}}\left(\left\|\mathbf{e}_{\mathbf{u}}\right\|_{L^{2}\left(K^{\prime}\right)}^{2}+\left\|\mathbf{o s c}_{K^{\prime}}^{\mathrm{st}}\right\|_{\boldsymbol{L}^{2}\left(K^{\prime}\right)}^{2}\right)\right) .
\end{aligned}
$$

On the other hand, using (3.5) and, again, integration by parts we obtain that

$$
\begin{aligned}
& \sum_{K \in \mathscr{T}}\left(\boldsymbol{\mathcal { R }}_{K}^{\mathrm{ad}}, \boldsymbol{\xi}\right)_{\boldsymbol{L}^{2}(K)}+\sum_{\gamma \in \mathcal{F}_{I}}\left(\llbracket \mathcal{R}_{\gamma}^{\mathrm{ad}} \rrbracket, \boldsymbol{\xi}\right)_{\boldsymbol{L}^{2}(\gamma)} \\
= & \mathcal{C}\left(\mathbf{e}_{\mathbf{w}}, \boldsymbol{\xi}\right)-\mathcal{B}\left(\boldsymbol{\xi}, \mathrm{e}_{\mathbf{q}}\right)-\left(\mathbf{e}_{\mathbf{y}}, \boldsymbol{\xi}\right)_{\boldsymbol{L}^{2}(\Omega)}-\sum_{K \in \mathscr{T}}\left(\mathbf{o s c}_{K}^{\mathrm{ad}}, \boldsymbol{\xi}\right)_{\boldsymbol{L}^{2}(K)} \quad \forall \boldsymbol{\xi} \in \mathbf{V} .
\end{aligned}
$$

Applying standard bubble function arguments, again, to this equation yields

$$
\left\|\boldsymbol{\mathcal { R }}_{K}^{\mathrm{ad}}\right\|_{\boldsymbol{L}^{2}(K)}^{2} \lesssim h_{K}^{-2}\left(\left\|\mathbf{e}_{\mathbf{w}}\right\|_{\mathbf{V}, K}^{2}+\varrho\left\|\mathrm{e}_{\mathbf{q}}\right\|_{Q, K}^{2}\right)+\left\|\mathbf{e}_{\mathbf{y}}\right\|_{\boldsymbol{L}^{2}(K)}^{2}+\left\|\mathbf{o s c}_{K}^{\mathrm{ad}}\right\|_{\boldsymbol{L}^{2}(K)}^{2}
$$

for $K \in \mathscr{T}$, and, for $\gamma \in \mathcal{F}_{I}$,

$$
\begin{aligned}
\left\|\llbracket \mathcal{R}_{\gamma}^{\mathrm{ad}} \rrbracket\right\|_{L^{2}(\gamma)}^{2} \lesssim & \sum_{K^{\prime} \in \Omega_{\gamma}}\left(h_{K^{\prime}}^{-1}\left(\left\|\mathbf{e}_{\mathbf{w}}\right\|_{\mathbf{V}, K^{\prime}}^{2}+\varrho\left\|\mathbf{e}_{\mathbf{q}}\right\|_{Q, K^{\prime}}^{2}\right)\right. \\
& \left.+h_{K^{\prime}}\left(\left\|\mathbf{e}_{\mathbf{y}}\right\|_{\boldsymbol{L}^{2}\left(K^{\prime}\right)}^{2}+\left\|\mathbf{o s c}_{K^{\prime}}^{\mathrm{ad}}\right\|_{\boldsymbol{L}^{2}\left(K^{\prime}\right)}^{2}\right)\right) .
\end{aligned}
$$

We now proceed to bound the terms $\left\|\nabla \cdot \overline{\mathbf{y}}_{\mathscr{T}}\right\|_{L^{2}(K)}^{2}$ and $\left\|\nabla \cdot \overline{\mathbf{w}}_{\mathscr{T}}\right\|_{L^{2}(K)}^{2}$ in (5.50). To accomplish this task, we notice that $\nabla \cdot \boldsymbol{\xi} \in Q$ for all $\boldsymbol{\xi} \in \mathbf{V}$. Then, it follows from the second equation of (3.7) that $\nabla \cdot \overline{\mathbf{y}}=0$, and thus that

$$
\left\|\nabla \cdot \overline{\mathbf{y}}_{\mathscr{T}}\right\|_{L^{2}(K)}^{2}=\left\|\nabla \cdot \mathbf{e}_{\mathbf{y}}\right\|_{L^{2}(K)}^{2} \lesssim\left\|\mathbf{e}_{\mathbf{y}}\right\|_{\mathbf{V}, K}^{2} \cdot
$$

Similarly, it follows from the fourth equation of (3.7) that

$$
\left\|\nabla \cdot \overline{\mathbf{w}}_{\mathscr{T}}\right\|_{L^{2}(K)}^{2}=\left\|\nabla \cdot \mathbf{e}_{\mathbf{w}}\right\|_{L^{2}(K)}^{2} \lesssim\left\|\mathbf{e}_{\mathbf{w}}\right\|_{\mathbf{V}, K}^{2} .
$$

We conclude with an estimate for the term $\eta_{\mathbf{u}, K}$ defined by (5.9):

$\eta_{\mathbf{u}, K} \leq\left\|\mathbf{e}_{\mathbf{u}}\right\|_{\boldsymbol{L}^{2}(K)}+\left\|\Pi_{[\mathbf{a}, \mathbf{b}]}\left(-\frac{1}{\vartheta} \overline{\mathbf{w}}\right)-\Pi_{[\mathbf{a}, \mathbf{b}]}\left(-\frac{1}{\vartheta} \overline{\mathbf{w}} \bar{T}\right)\right\|_{\boldsymbol{L}^{2}(K)} \leq\left\|\mathbf{e}_{\mathbf{u}}\right\|_{\boldsymbol{L}^{2}(K)}+\frac{1}{\vartheta}\left\|\mathbf{e}_{\mathbf{w}}\right\|_{\boldsymbol{L}^{2}(K)}$ upon invoking the triangle inequality, (3.8), and (3.9). Hence,

$$
\eta_{\mathbf{u}, K}^{2} \lesssim\left\|\mathbf{e}_{\mathbf{u}}\right\|_{\boldsymbol{L}^{2}(K)}^{2}+\left\|\mathbf{e}_{\mathbf{w}}\right\|_{\boldsymbol{L}^{2}(K)}^{2} .
$$

The following theorem then follows upon combining (5.50)-(5.57). 
Theorem 5.6 (local efficiency). If $\varrho \neq 0$ and Assumptions 3, 4, 5, 6 and 7 hold, then

$$
\begin{aligned}
\Upsilon_{K}^{2} \lesssim \tilde{\Upsilon}_{K}^{2} \lesssim & \left\|\mathbf{e}_{\mathbf{w}}\right\|_{L^{2}(K)}^{2}+\sum_{K^{\prime} \in \tilde{\Omega}_{K}}\left(\left\|\left(\mathbf{e}_{\mathbf{y}}, \mathrm{e}_{\mathbf{p}}, \mathbf{e}_{\mathbf{w}}, \mathbf{e}_{\mathbf{q}}, \mathbf{e}_{\mathbf{u}}\right)\right\|_{K^{\prime}}^{2}\right. \\
& \left.+h_{K^{\prime}}^{2}\left(\left\|\mathbf{e}_{\mathbf{u}}\right\|_{\boldsymbol{L}^{2}\left(K^{\prime}\right)}^{2}+\left\|\mathbf{e}_{\mathbf{y}}\right\|_{\boldsymbol{L}^{2}\left(K^{\prime}\right)}^{2}+\left\|\operatorname{osc}_{K^{\prime}}^{\mathrm{st}}\right\|_{\boldsymbol{L}^{2}\left(K^{\prime}\right)}^{2}+\left\|\operatorname{osc}_{K^{\prime}}^{\mathrm{ad}}\right\|_{\boldsymbol{L}^{2}\left(K^{\prime}\right)}^{2}\right)\right)
\end{aligned}
$$

with $\tilde{\Omega}_{K}=\hat{\mathscr{T}}_{K} \cup \bigcup_{\gamma \in \hat{\mathcal{F}}_{K}} \Omega_{\gamma}$.

The following corollary follows upon using (2.6) and the fact that $\Omega$ is bounded.

Corollary 5.7 (global efficiency). If $\varrho \neq 0$ and Assumptions 3, 4, 5, 6 and 7 hold, then

$$
\sum_{K \in \mathscr{T}} \Upsilon_{K}^{2} \lesssim \tilde{\Upsilon}^{2} \lesssim\left\|\left(\mathbf{e}_{\mathbf{y}}, \mathrm{e}_{\mathbf{p}}, \mathbf{e}_{\mathbf{w}}, \mathrm{e}_{\mathbf{q}}, \mathbf{e}_{\mathbf{u}}\right)\right\|_{\Omega}^{2}+\sum_{K \in \mathscr{T}} h_{K}^{2}\left(\left\|\mathbf{o s c}_{K}^{\mathrm{st}}\right\|_{\boldsymbol{L}^{2}(K)}^{2}+\left\|\mathbf{o s c}_{K}^{\mathrm{ad}}\right\|_{\boldsymbol{L}^{2}(K)}^{2}\right) .
$$

6. A particular example. Henceforth, we shall consider a particular case of the approximation scheme (4.1). We set $\mathbf{V}(\mathscr{T})=\mathbf{V} \cap \mathbb{P}_{1}(\mathscr{T}), Q(\mathscr{T})=Q \cap \mathbb{P}_{0}(\mathscr{T})$, $\mathbf{U}_{a d}(\mathscr{T})=\mathbf{U}_{a d} \cap \mathbb{P}_{0}(\mathscr{T})$,

$$
\mathcal{Q}\left(\overline{\mathbf{w}}_{\mathscr{T}}, \overline{\mathrm{q}}_{\mathscr{T}}, \overline{\mathbf{y}}_{\mathscr{T}}-\mathbf{y}_{\Omega} ; \zeta\right)=\sum_{K \in \mathscr{T}} \mathcal{Q}_{K}\left(\overline{\mathbf{w}}_{\mathscr{T}}, \overline{\mathrm{q}}_{\mathscr{T}}, \overline{\mathbf{y}}_{\mathscr{T}}-\mathbf{y}_{\Omega} ; \boldsymbol{\zeta}\right)
$$

and

$$
\mathcal{K}\left(\overline{\mathbf{w}}_{\mathscr{T}}, \overline{\mathbf{q}}_{\mathscr{T}}, \overline{\mathbf{y}}_{\mathscr{T}}-\mathbf{y}_{\Omega} ; \psi\right)=-\tau_{\gamma} \sum_{\gamma \in \mathcal{F}_{I}} h_{\gamma}\left(\left[\overline{\mathbf{q}}_{\mathscr{T}}\right],[\psi]\right)_{L^{2}(\gamma)},
$$

where

$$
\begin{gathered}
\mathcal{S}_{K}\left(\overline{\mathbf{y}}_{\mathscr{T}}, \overline{\mathbf{p}}_{\mathscr{T}}, \mathbf{f}+\overline{\mathbf{u}}_{\mathscr{T}} ; \boldsymbol{\xi}\right)=\tau_{K}\left((\mathbf{c} \cdot \nabla) \overline{\mathbf{y}}_{\mathscr{T}}+\kappa \overline{\mathbf{y}}_{\mathscr{T}}-\left(\mathbf{f}+\overline{\mathbf{u}}_{\mathscr{T}}\right),(\mathbf{c} \cdot \nabla) \boldsymbol{\xi}\right)_{\boldsymbol{L}^{2}(K)}, \\
\mathcal{Q}_{K}\left(\overline{\mathbf{w}}_{\mathscr{T}}, \overline{\mathbf{q}}_{\mathscr{T}}, \overline{\mathbf{y}}_{\mathscr{T}}-\mathbf{y}_{\Omega} ; \boldsymbol{\zeta}\right)=\tau_{K}\left((\mathbf{c} \cdot \nabla) \overline{\mathbf{w}}_{\mathscr{T}}-\kappa \overline{\mathbf{w}}_{\mathscr{T}}+\overline{\mathbf{y}}_{\mathscr{T}}-\mathbf{y}_{\Omega},(\mathbf{c} \cdot \nabla) \boldsymbol{\zeta}\right)_{\boldsymbol{L}^{2}(K)}
\end{gathered}
$$

and $[v]$ denotes the jumps in $v$. The stabilization parameters $\tau_{\gamma}$ and $\tau_{K}$ are such that $\tau_{\gamma}>0$ and $0<\tau_{K} \lesssim h_{K}^{2}$. Note that these choices correspond to solving the state equations using a particular case of the method given by [38, equation (3.6)] and are such that Assumption 6 is satisfied.

We note that alternative methods for solving the state equations can be found in [17] but we restrict our attention to the method described above in order to simplify the presentation. 
6.1. Fully computable a posteriori error estimators. In this section we obtain a posteriori error estimators that satisfy the assumptions of Section 5 and are fully computable if the value of a $\beta$ satisfying (2.14) is known. We first define some quantities that we will make use of.

For $\varsigma=$ st and $\varsigma=$ ad, let the equilibrated fluxes $\boldsymbol{g}_{\gamma, K}^{\varsigma} \in \mathbb{P}_{1}(\gamma)^{d}$ be such that

$$
\begin{gathered}
\boldsymbol{g}_{\gamma, K}^{\varsigma}+\boldsymbol{g}_{\gamma, K^{\prime}}^{\varsigma}=0, \text { if } \gamma \in \mathcal{F}_{K} \cap \mathcal{F}_{K^{\prime}}, K, K^{\prime} \in \mathscr{T}, K \neq K^{\prime}, \\
\left(\mathbf{f}+\overline{\mathbf{u}}_{\mathscr{T}}, \boldsymbol{\lambda}\right)_{\boldsymbol{L}^{2}(K)}-\varepsilon\left(\nabla \overline{\mathbf{y}}_{\mathscr{T}}, \nabla \boldsymbol{\lambda}\right)_{\underset{\sim}{\boldsymbol{L}^{2}(K)}}-\left(\kappa \overline{\mathbf{y}}_{\mathscr{T}}+(\mathbf{c} \cdot \nabla) \overline{\mathbf{y}}_{\mathscr{T}}, \boldsymbol{\lambda}\right)_{\boldsymbol{L}^{2}(K)} \\
+\left(\overline{\mathbf{p}}_{\mathscr{T}}, \nabla \cdot \boldsymbol{\lambda}\right)_{L^{2}(K)}-\mathcal{S}_{K}\left(\overline{\mathbf{y}}_{\mathscr{T}}, \overline{\mathbf{p}} \mathscr{T}, \mathbf{f}+\overline{\mathbf{u}}_{\mathscr{T}} ; \boldsymbol{\lambda}\right)+\sum_{\gamma \in \mathcal{F}_{K}}\left(\boldsymbol{g}_{\gamma, K}^{\mathrm{st}}, \boldsymbol{\lambda}\right)_{\boldsymbol{L}^{2}(\gamma)}=0
\end{gathered}
$$

for all $\boldsymbol{\lambda} \in \mathbb{P}_{1}(K)^{d}$ and all $K \in \mathcal{P}$,

$$
\begin{aligned}
& \left(\overline{\mathbf{y}}_{\mathscr{T}}-\mathbf{y}_{\Omega}, \boldsymbol{\lambda}\right)_{\boldsymbol{L}^{2}(K)}-\varepsilon\left(\nabla \overline{\mathbf{w}}_{\mathscr{T}}, \nabla \boldsymbol{\lambda}\right)_{\underset{\sim}{L^{2}(K)}}-\left(\kappa \overline{\mathbf{w}}_{\mathscr{T}}-(\mathbf{c} \cdot \nabla) \overline{\mathbf{w}}_{\mathscr{T}}, \boldsymbol{\lambda}\right)_{\boldsymbol{L}^{2}(K)} \\
& \quad-\left(\overline{\mathrm{q}}_{\mathscr{T}}, \nabla \cdot \boldsymbol{\lambda}\right)_{L^{2}(K)}-\mathcal{Q}_{K}\left(\overline{\mathbf{w}}_{\mathscr{T}}, \overline{\mathrm{q}}_{\mathscr{T}}, \overline{\mathbf{y}}_{\mathscr{T}}-\mathbf{y}_{\Omega} ; \boldsymbol{\lambda}\right)+\sum_{\gamma \in \mathcal{F}_{K}}\left(\boldsymbol{g}_{\gamma, K}^{\mathrm{ad}}, \boldsymbol{\lambda}\right)_{\boldsymbol{L}^{2}(\gamma)}=0
\end{aligned}
$$

for all $\boldsymbol{\lambda} \in \mathbb{P}_{1}(K)^{d}$ and all $K \in \mathcal{P}$, and

$$
\sum_{\gamma \in \mathcal{F}_{K}} h_{K}\left\|\boldsymbol{g}_{\gamma, K}^{\varsigma}+\boldsymbol{\mathcal { R }}_{\gamma, K}^{\varsigma}\right\|_{\boldsymbol{L}^{2}(\gamma)}^{2} \lesssim \sum_{K^{\prime} \in \hat{\mathscr{T}}_{K}} h_{K}^{2}\left\|\boldsymbol{\mathcal { R }}_{K^{\prime}}^{\varsigma}\right\|_{\boldsymbol{L}^{2}\left(K^{\prime}\right)}^{2}+\sum_{\gamma \in \hat{\mathcal{F}}_{K}} h_{K}\left\|\llbracket \boldsymbol{\mathcal { R }}_{\gamma}^{\varsigma} \rrbracket\right\|_{\boldsymbol{L}^{2}(\gamma)}^{2}
$$

for all $K \in \mathcal{P}$, where

$$
\hat{\mathscr{T}}_{K}=\left\{K^{\prime} \in \mathscr{T}: \mathcal{V}_{K} \cap \mathcal{V}_{K^{\prime}} \neq \emptyset\right\} \quad \text { and } \quad \hat{\mathcal{F}}_{K}=\bigcup_{\gamma \in \mathcal{F}_{\mathcal{K}}}\left\{\gamma^{\prime} \in \mathcal{F}_{I}: \mathcal{V}_{\gamma} \cap \mathcal{V}_{\gamma^{\prime}} \neq \emptyset\right\}
$$

with $\mathcal{V}_{K}$ denoting the set containing the vertices of element $K$ and $\mathcal{V}_{\gamma}$ denoting the set containing the vertices of the edge/face $\gamma$. For information that will help with the construction of such $\boldsymbol{g}_{\gamma, K}^{\varsigma}$ we refer the reader to [3, Chapter 6] and [5, 6].

For $\varsigma=$ st and $\varsigma=$ ad, we also define $\boldsymbol{\sigma}_{K}^{\varsigma} \in \mathbb{P}_{2}(K)^{d \times d}$ to be such that

$$
\left\{\begin{array}{l}
-\operatorname{div} \boldsymbol{\sigma}_{K}^{\varsigma}=\boldsymbol{\mathcal { R }}_{K}^{\varsigma} \text { in } K, \\
\boldsymbol{\sigma}_{K}^{\varsigma} \boldsymbol{n}_{\gamma}^{K}=\boldsymbol{g}_{\gamma, K}^{\varsigma}+\boldsymbol{\mathcal { R }}_{\gamma, K}^{\varsigma} \text { on } \gamma, \forall \gamma \in \mathcal{F}_{K},
\end{array}\right.
$$

and $\left\|\boldsymbol{\sigma}_{K}^{\varsigma}\right\|_{\boldsymbol{\sim}^{2}(K)}$ is minimized. We note that the $\boldsymbol{g}_{\gamma, K}^{\varsigma}$ are such that the data in the above problem are compatible in the sense that $\boldsymbol{\sigma}_{K}^{\varsigma}$ exists. Moreover, for all $K \in \mathscr{T}$,

$$
\left(\boldsymbol{\sigma}_{K}^{\varsigma}, \nabla \boldsymbol{\xi}\right)_{\boldsymbol{\sim}^{2}(K)}=\left(\boldsymbol{\mathcal { R }}_{K}^{\varsigma}, \boldsymbol{\xi}\right)_{\boldsymbol{L}^{2}(K)}+\sum_{\gamma \in \mathcal{F}_{K}}\left(\boldsymbol{g}_{\gamma, K}^{\varsigma}+\boldsymbol{\mathcal { R }}_{\gamma, K}^{\varsigma}, \boldsymbol{\xi}\right)_{\boldsymbol{L}^{2}(\gamma)} \forall \boldsymbol{\xi} \in \mathbf{V}
$$

and

$$
\left\|\boldsymbol{\sigma}_{K}^{\varsigma}\right\|_{\boldsymbol{\sim}^{2}(K)}^{2} \lesssim h_{K}^{2}\left\|\boldsymbol{\mathcal { R }}_{K^{\prime}}^{\varsigma}\right\|_{\boldsymbol{L}^{2}(K)}^{2}+\sum_{\gamma \in \mathcal{F}_{K}} h_{K}\left\|\boldsymbol{g}_{\gamma, K}^{\varsigma}+\boldsymbol{\mathcal { R }}_{\gamma, K}^{\varsigma}\right\|_{\boldsymbol{L}^{2}(\gamma)}^{2} .
$$

For information on the construction of such $\boldsymbol{\sigma}_{K}^{\varsigma}$ we refer the reader to $[4,5]$.

Finally, for $\varsigma=$ st and $\varsigma=$ ad, we define

$$
\Psi_{\varsigma, K}=\frac{1}{\sqrt{\varepsilon}}\left\|\boldsymbol{\sigma}_{K}^{\varsigma}\right\|_{\boldsymbol{L}^{2}(K)}+\mathrm{C}_{K}\left\|\operatorname{osc}_{K}^{\varsigma}\right\|_{\boldsymbol{L}^{2}(K)} .
$$

We thus have the following result. 
Theorem 6.1. Assumption 3 holds with

$$
\eta_{\mathbf{y}, K}^{2}=3 \Psi_{\mathrm{st}, K}^{2}+\mathrm{C}_{\mathrm{is}}^{2}\left(1+2 \mathrm{C}_{\mathrm{ct}}^{2}\right)\left\|\nabla \cdot \overline{\mathbf{y}}_{\mathscr{T}}\right\|_{L^{2}(K)}^{2}
$$

and

$$
\eta_{\mathrm{p}, K}^{2}=2 \mathrm{C}_{\mathrm{is}}^{2}\left(\left(1+3 \mathrm{C}_{\mathrm{ct}}^{2}\right) \Psi_{\mathrm{st}, K}^{2}+\mathrm{C}_{\mathrm{is}}^{2} \mathrm{C}_{\mathrm{ct}}^{2}\left(1+2 \mathrm{C}_{\mathrm{ct}}^{2}\right)\left\|\nabla \cdot \overline{\mathbf{y}}_{\mathscr{T}}\right\|_{L^{2}(K)}^{2}\right) .
$$

Moreover, Assumption 1 holds with

$$
\eta_{\mathbf{y}}=\left(\sum_{K \in \mathcal{P}} \eta_{\mathbf{y}, K}^{2}\right)^{1 / 2} \text { and } \eta_{\mathrm{p}}=\left(\sum_{K \in \mathcal{P}} \eta_{\mathrm{p}, K}^{2}\right)^{1 / 2} .
$$

Proof. Let $\boldsymbol{E}_{\mathbf{y}} \in \mathbf{V}$ be the solution to

$$
\varepsilon\left(\nabla \boldsymbol{E}_{\mathbf{y}}, \nabla \boldsymbol{\xi}\right)_{\boldsymbol{\sim}^{2}(\Omega)}+\kappa\left(\boldsymbol{E}_{\mathbf{y}}, \boldsymbol{\xi}\right)_{\boldsymbol{L}^{2}(\Omega)}=\mathcal{A}\left(\hat{\mathbf{y}}-\overline{\mathbf{y}}_{\mathscr{T}}, \boldsymbol{\xi}\right)-\mathcal{B}(\boldsymbol{\xi}, \hat{\mathrm{p}}-\overline{\mathbf{p}} \mathscr{T}) \quad \forall \boldsymbol{\xi} \in \mathbf{V}
$$

Letting $\phi=\hat{\mathrm{p}}-\overline{\mathrm{p}}_{\mathscr{T}}$ in (2.15) yields that

$$
\left\|\hat{\mathrm{p}}-\overline{\mathrm{p}}_{\mathscr{T}}\right\|_{Q, \Omega} \leq \mathrm{C}_{\text {is }} \sup _{\boldsymbol{\xi} \in \mathbf{V} \backslash\{\mathbf{0}\}} \frac{\mathcal{B}\left(\boldsymbol{\xi}, \hat{\mathrm{p}}-\overline{\mathrm{p}}_{\mathscr{T}}\right)}{\|\boldsymbol{\xi}\|_{\mathbf{V}, \Omega}} .
$$

To control the right-hand side of the previous estimate we use (6.13) and obtain that

$$
\begin{aligned}
\mathcal{B}(\boldsymbol{\xi}, \hat{\mathrm{p}}-\overline{\mathrm{p}} \mathscr{T}) & =\mathcal{A}\left(\hat{\mathbf{y}}-\overline{\mathbf{y}}_{\mathscr{T}}, \boldsymbol{\xi}\right)-\varepsilon\left(\nabla \boldsymbol{E}_{\mathbf{y}}, \nabla \boldsymbol{\xi}\right)_{\underset{\sim}{\boldsymbol{L}^{2}(\Omega)}}-\kappa\left(\boldsymbol{E}_{\mathbf{y}}, \boldsymbol{\xi}\right)_{\boldsymbol{L}^{2}(\Omega)} \\
& \leq \mathrm{C}_{\mathrm{ct}}\left\|\hat{\mathbf{y}}-\overline{\mathbf{y}}_{\mathscr{T}}\right\|_{\mathbf{V}, \Omega}\|\boldsymbol{\xi}\|_{\mathbf{V}, \Omega}+\left\|\boldsymbol{E}_{\mathbf{y}}\right\|_{\mathbf{V}, \Omega}\|\boldsymbol{\xi}\|_{\mathbf{V}, \Omega},
\end{aligned}
$$

upon using (2.12). Hence,

$$
\|\hat{\mathbf{p}}-\overline{\mathbf{p}} \mathscr{T}\|_{Q, \Omega} \leq \mathrm{C}_{\text {is }}\left(\left\|\boldsymbol{E}_{\mathbf{y}}\right\|_{\mathbf{V}, \Omega}+\mathrm{C}_{\mathrm{ct}}\left\|\hat{\mathbf{y}}-\overline{\mathbf{y}}_{\mathscr{T}}\right\|_{\mathbf{V}, \Omega}\right) .
$$

We now estimate $\left\|\hat{\mathbf{y}}-\overline{\mathbf{y}}_{\mathscr{T}}\right\|_{\mathbf{V}, \Omega}$. Since $\hat{\mathbf{p}}-\overline{\mathbf{p}} \mathscr{T} \in Q$, by using the second equation of (5.2) we have that

$$
\mathcal{B}\left(\hat{\mathbf{y}}-\overline{\mathbf{y}}_{\mathscr{T}}, \hat{\mathbf{p}}-\overline{\mathbf{p}}_{\mathscr{T}}\right)=-\mathcal{B}\left(\overline{\mathbf{y}}_{\mathscr{T}}, \hat{\mathbf{p}}-\overline{\mathbf{p}} \mathscr{T}\right) \leq\left\|\nabla \cdot \overline{\mathbf{y}}_{\mathscr{T}}\right\|_{L^{2}(\Omega)}\left\|\hat{\mathbf{p}}-\overline{\mathbf{p}}_{\mathscr{T}}\right\|_{Q, \Omega}
$$

Thus, by using the previous estimate and letting $\boldsymbol{\xi}=\hat{\mathbf{y}}-\overline{\mathbf{y}}_{\mathscr{T}}$ in (6.13), we arrive at

$$
\begin{aligned}
\left\|\hat{\mathbf{y}}-\overline{\mathbf{y}}_{\mathscr{T}}\right\|_{\mathbf{V}, \Omega}^{2} & =\varepsilon\left(\nabla \boldsymbol{E}_{\mathbf{y}}, \nabla\left(\hat{\mathbf{y}}-\overline{\mathbf{y}}_{\mathscr{T}}\right)\right)_{{\underset{\sim}{2}}^{2}(\Omega)}+\kappa\left(\boldsymbol{E}_{\mathbf{y}}, \hat{\mathbf{y}}-\overline{\mathbf{y}}_{\mathscr{T}}\right)_{L^{2}(\Omega)}+\mathcal{B}\left(\hat{\mathbf{y}}-\overline{\mathbf{y}}_{\mathscr{T}}, \hat{\mathbf{p}}-\overline{\mathbf{p}}_{\mathscr{T}}\right) \\
& \leq\left\|\boldsymbol{E}_{\mathbf{y}}\right\|_{\mathbf{V}, \Omega}\left\|\hat{\mathbf{y}}-\overline{\mathbf{y}}_{\mathscr{T}}\right\|_{\mathbf{V}, \Omega}+\left\|\nabla \cdot \overline{\mathbf{y}}_{\mathscr{T}}\right\|_{L^{2}(\Omega)}\left\|\hat{\mathbf{p}}-\overline{\mathbf{p}}_{\mathscr{T}}\right\|_{Q, \Omega} .
\end{aligned}
$$

This, in view of (6.14), then yields that

$$
\begin{aligned}
\left\|\hat{\mathbf{y}}-\overline{\mathbf{y}}_{\mathscr{T}}\right\|_{\mathbf{V}, \Omega}^{2} \leq & \mathrm{C}_{\mathrm{is}}\left\|\nabla \cdot \overline{\mathbf{y}}_{\mathscr{T}}\right\|_{L^{2}(\Omega)}\left\|\boldsymbol{E}_{\mathbf{y}}\right\|_{\mathbf{V}, \Omega} \\
& +\left(\left\|\boldsymbol{E}_{\mathbf{y}}\right\|_{\mathbf{V}, \Omega}+\mathrm{C}_{\mathrm{is}} \mathrm{C}_{\mathrm{ct}}\left\|\nabla \cdot \overline{\mathbf{y}}_{\mathscr{T}}\right\|_{L^{2}(\Omega)}\right)\left\|\hat{\mathbf{y}}-\overline{\mathbf{y}}_{\mathscr{T}}\right\|_{\mathbf{V}, \Omega} \\
\leq & \frac{\mathrm{C}_{\mathrm{is}}^{2}}{2}\left\|\nabla \cdot \overline{\mathbf{y}}_{\mathscr{T}}\right\|_{L^{2}(\Omega)}^{2}+\frac{1}{2}\left\|\boldsymbol{E}_{\mathbf{y}}\right\|_{\mathbf{V}, \Omega}^{2} \\
& +\frac{1}{2}\left(\left\|\boldsymbol{E}_{\mathbf{y}}\right\|_{\mathbf{V}, \Omega}+\mathrm{C}_{\mathrm{is}} \mathrm{C}_{\mathrm{ct}}\left\|\nabla \cdot \overline{\mathbf{y}}_{\mathscr{T}}\right\|_{L^{2}(\Omega)}\right)^{2}+\frac{1}{2}\left\|\hat{\mathbf{y}}-\overline{\mathbf{y}}_{\mathscr{T}}\right\|_{\mathbf{V}, \Omega}^{2}
\end{aligned}
$$


from which it follows that

$$
\left\|\hat{\mathbf{y}}-\overline{\mathbf{y}}_{\mathscr{T}}\right\|_{\mathbf{V}, \Omega}^{2} \leq \mathrm{C}_{\mathrm{is}}^{2}\left\|\nabla \cdot \overline{\mathbf{y}}_{\mathscr{T}}\right\|_{L^{2}(\Omega)}^{2}+\left\|\boldsymbol{E}_{\mathbf{y}}\right\|_{\mathbf{V}, \Omega}^{2}+\left(\left\|\boldsymbol{E}_{\mathbf{y}}\right\|_{\mathbf{V}, \Omega}+\mathrm{C}_{\mathrm{is}} \mathrm{C}_{\mathrm{ct}}\left\|\nabla \cdot \overline{\mathbf{y}}_{\mathscr{T}}\right\|_{L^{2}(\Omega)}\right)^{2} .
$$

Hence, upon observing that

$$
\left(\left\|\boldsymbol{E}_{\mathbf{y}}\right\|_{\mathbf{V}, \Omega}+\mathrm{C}_{\mathrm{is}} \mathrm{C}_{\mathrm{ct}}\left\|\nabla \cdot \overline{\mathbf{y}}_{\mathscr{T}}\right\|_{L^{2}(\Omega)}\right)^{2} \leq 2\left\|\boldsymbol{E}_{\mathbf{y}}\right\|_{\mathbf{V}, \Omega}^{2}+2 \mathrm{C}_{\mathrm{is}}^{2} \mathrm{C}_{\mathrm{ct}}^{2}\left\|\nabla \cdot \overline{\mathbf{y}}_{\mathscr{T}}\right\|_{L^{2}(\Omega)}^{2},
$$

we can arrive at

$$
\left\|\hat{\mathbf{y}}-\overline{\mathbf{y}}_{\mathscr{T}}\right\|_{\mathbf{V}, \Omega}^{2} \leq 3\left\|\boldsymbol{E}_{\mathbf{y}}\right\|_{\mathbf{V}, \Omega}^{2}+\mathrm{C}_{\text {is }}^{2}\left(1+2 \mathrm{C}_{\mathrm{ct}}^{2}\right)\left\|\nabla \cdot \overline{\mathbf{y}}_{\mathscr{T}}\right\|_{L^{2}(\Omega)}^{2} .
$$

Furthermore, (6.14) allows us to conclude that

$$
\left\|\hat{\mathrm{p}}-\overline{\mathrm{p}}_{\mathscr{T}}\right\|_{Q, \Omega}^{2} \leq 2 \mathrm{C}_{\mathrm{is}}^{2}\left(\left\|\boldsymbol{E}_{\mathbf{y}}\right\|_{\mathbf{V}, \Omega}^{2}+\mathrm{C}_{\mathrm{ct}}^{2}\left\|\hat{\mathbf{y}}-\overline{\mathbf{y}}_{\mathscr{T}}\right\|_{\mathbf{V}, \Omega}^{2}\right) .
$$

Applying (6.15) then yields that

$$
\left\|\hat{\mathrm{p}}-\overline{\mathrm{p}}_{\mathscr{T}}\right\|_{Q, \Omega}^{2} \leq 2 \mathrm{C}_{\mathrm{is}}^{2}\left(\left(1+3 \mathrm{C}_{\mathrm{ct}}^{2}\right)\left\|\boldsymbol{E}_{\mathbf{y}}\right\|_{\mathrm{V}, \Omega}^{2}+\mathrm{C}_{\mathrm{is}}^{2} \mathrm{C}_{\mathrm{ct}}^{2}\left(1+2 \mathrm{C}_{\mathrm{ct}}^{2}\right)\left\|\nabla \cdot \overline{\mathbf{y}}_{\mathscr{T}}\right\|_{L^{2}(\Omega)}^{2}\right) .
$$

Now, letting $\boldsymbol{\xi}=\boldsymbol{E}_{\mathbf{y}}$ in (6.13) yields that

$$
\begin{aligned}
\left\|\boldsymbol{E}_{\mathbf{y}}\right\|_{\mathbf{V}, \Omega}^{2} & =\mathcal{A}\left(\hat{\mathbf{y}}-\overline{\mathbf{y}}_{\mathscr{T}}, \boldsymbol{E}_{\mathbf{y}}\right)-\mathcal{B}\left(\boldsymbol{E}_{\mathbf{y}}, \hat{\mathrm{p}}-\overline{\mathrm{p}}_{\mathscr{T}}\right) \\
& =\sum_{K \in \mathscr{T}}\left(\left(\boldsymbol{\mathcal { R }}_{K}^{\mathrm{st}}, \boldsymbol{E}_{\mathbf{y}}\right)_{\boldsymbol{L}^{2}(K)}+\sum_{\gamma \in \mathcal{F}_{K}}\left(\boldsymbol{g}_{\gamma, K}^{\mathrm{st}}+\boldsymbol{\mathcal { R }}_{\gamma, K}^{\mathrm{st}}, \boldsymbol{E}_{\mathbf{y}}\right)_{\boldsymbol{L}^{2}(\gamma)}+\left(\mathbf{o s c}_{K}^{\mathrm{st}}, \boldsymbol{E}_{\mathbf{y}}\right)_{\boldsymbol{L}^{2}(K)}\right)
\end{aligned}
$$

by (5.2), integration by parts, (5.44), (5.46), (5.48) and (6.5). Applying (6.7) and (2.3) then yields that

$$
\begin{aligned}
\left\|\boldsymbol{E}_{\mathbf{y}}\right\|_{\mathbf{V}, \Omega}^{2} & =\sum_{K \in \mathscr{T}}\left(\left(\boldsymbol{\sigma}_{K}^{\mathrm{st}}, \nabla \boldsymbol{E}_{\mathbf{y}}\right)_{\underset{\sim}{L^{2}(K)}}+\left(\mathbf{o s c}_{K}^{\mathbf{s t}}, \boldsymbol{E}_{\mathbf{y}}-\Pi_{K, 0}\left(\boldsymbol{E}_{\mathbf{y}}\right)\right)_{\boldsymbol{L}^{2}(K)}\right) \\
& \leq\left(\sum_{K \in \mathscr{T}} \Psi_{\mathbf{s t}, K}^{2}\right)^{1 / 2}\left\|\boldsymbol{E}_{\mathbf{y}}\right\|_{\mathbf{V}, \Omega}
\end{aligned}
$$

by the Cauchy-Schwarz inequality and (2.4). Consequently,

$$
\left\|\boldsymbol{E}_{\mathbf{y}}\right\|_{\mathbf{V}, \Omega}^{2} \leq \sum_{K \in \mathscr{T}} \Psi_{\text {st }, K}^{2}
$$

The theorem then follows upon combining (6.15), (6.16) and (6.17).

We note that the above theorem is an improvement and adaptation to the case considered in this section of the results from [5]. The below theorem can be proved similarly to how the above theorem was proved.

TheOREM 6.2. Assumption 4 holds with

$$
\eta_{\mathbf{w}, K}^{2}=3 \Psi_{\mathrm{ad}, K}^{2}+\mathrm{C}_{\text {is }}^{2}\left(1+2 \mathrm{C}_{\mathrm{ct}}^{2}\right)\left\|\nabla \cdot \overline{\mathbf{w}}_{\mathscr{T}}\right\|_{L^{2}(K)}^{2}
$$


and

$$
\eta_{\mathrm{q}, K}^{2}=2 \mathrm{C}_{\mathrm{is}}^{2}\left(\left(1+3 \mathrm{C}_{\mathrm{ct}}^{2}\right) \Psi_{\mathrm{ad}, K}^{2}+\mathrm{C}_{\mathrm{is}}^{2} \mathrm{C}_{\mathrm{ct}}^{2}\left(1+2 \mathrm{C}_{\mathrm{ct}}^{2}\right)\left\|\nabla \cdot \overline{\mathbf{w}}_{\mathscr{T}}\right\|_{L^{2}(K)}^{2}\right) .
$$

Moreover, Assumption 2 holds with

$$
\eta_{\mathbf{w}}=\left(\sum_{K \in \mathcal{P}} \eta_{\mathbf{w}, K}^{2}\right)^{1 / 2} \text { and } \eta_{\mathbf{q}}=\left(\sum_{K \in \mathcal{P}} \eta_{\mathbf{q}, K}^{2}\right)^{1 / 2} \text {. }
$$

We note that, if the value of a $\beta$ satisfying (2.14) is known, then Assumption 5 holds with $\tilde{\eta}_{\mathbf{y}, K}=\eta_{\mathbf{y}, K}, \tilde{\eta}_{\mathrm{p}, K}=\eta_{\mathrm{p}, K}, \tilde{\eta}_{\mathbf{w}, K}=\eta_{\mathbf{w}, K}$ and $\tilde{\eta}_{\mathbf{q}, K}=\eta_{\mathbf{q}, K}$. Furthermore, by (6.6) and (6.8) we have that

$$
\begin{aligned}
\eta_{\mathbf{y}, K}^{2}+\eta_{\mathbf{p}, K}^{2} \lesssim & \left\|\nabla \cdot \overline{\mathbf{y}}_{\mathscr{T}}\right\|_{L^{2}(K)}^{2}+\sum_{\gamma \in \hat{\mathcal{F}}_{K}} h_{K}\left\|\llbracket \boldsymbol{\mathcal { R }}_{\gamma}^{\mathrm{st}} \rrbracket\right\|_{\boldsymbol{L}^{2}(\gamma)}^{2} \\
& +\sum_{K^{\prime} \in \hat{\mathscr{T}}_{K}} h_{K}^{2}\left(\left\|\boldsymbol{\mathcal { R }}_{K^{\prime}}^{\mathrm{st}}\right\|_{\boldsymbol{L}^{2}\left(K^{\prime}\right)}^{2}+\left\|\mathbf{o s c}_{K^{\prime}}^{\mathrm{st}}\right\|_{\boldsymbol{L}^{2}\left(K^{\prime}\right)}^{2}\right)
\end{aligned}
$$

and

$$
\begin{aligned}
\eta_{\mathbf{w}, K}^{2}+\eta_{\mathbf{q}, K}^{2} \lesssim & \left\|\nabla \cdot \overline{\mathbf{w}}_{\mathscr{T}}\right\|_{L^{2}(K)}^{2}+\sum_{\gamma \in \hat{\mathcal{F}}_{K}} h_{K}\left\|\llbracket \mathcal{R}_{\gamma}^{\mathrm{ad}} \rrbracket\right\|_{\boldsymbol{L}^{2}(\gamma)}^{2} \\
& +\sum_{K^{\prime} \in \hat{\mathscr{T}}_{K}} h_{K}^{2}\left(\left\|\boldsymbol{\mathcal { R }}_{K^{\prime}}^{\mathrm{ad}}\right\|_{\boldsymbol{L}^{2}\left(K^{\prime}\right)}^{2}+\left\|\mathbf{o s c}_{K^{\prime}}^{\mathrm{ad}}\right\|_{\boldsymbol{L}^{2}\left(K^{\prime}\right)}^{2}\right)
\end{aligned}
$$

from which it follows that Assumption $\mathbf{7}$ is also satisfied. We note that it also follows that

$$
\begin{aligned}
\left\|\hat{\mathbf{y}}-\overline{\mathbf{y}}_{\mathscr{T}}\right\|_{\mathbf{V}, \Omega}^{2}+\|\hat{\mathbf{p}}-\overline{\mathrm{p}} \mathscr{T}\|_{Q, \Omega}^{2} \lesssim & \sum_{K \in \mathscr{T}}\left(\left\|\nabla \cdot \overline{\mathbf{y}}_{\mathscr{T}}\right\|_{L^{2}(K)}^{2}+\sum_{\gamma \in \mathcal{F}_{K}} h_{K}\left\|\llbracket \mathcal{R}_{\gamma}^{\mathrm{st}} \rrbracket\right\|_{\boldsymbol{L}^{2}(\gamma)}^{2}\right. \\
& \left.+h_{K}^{2}\left(\left\|\boldsymbol{\mathcal { R }}_{K}^{\mathrm{st}}\right\|_{\boldsymbol{L}^{2}(K)}^{2}+\left\|\mathbf{o s c}_{K}^{\mathrm{st}}\right\|_{\boldsymbol{L}^{2}(K)}^{2}\right)\right)
\end{aligned}
$$

and

$$
\begin{aligned}
\left\|\hat{\mathbf{w}}-\overline{\mathbf{w}}_{\mathscr{T}}\right\|_{\mathbf{V}, \Omega}^{2}+\left\|\hat{\mathbf{q}}-\overline{\mathbf{q}}_{\mathscr{T}}\right\|_{Q, \Omega}^{2} \lesssim & \sum_{K \in \mathscr{T}}\left(\left\|\nabla \cdot \overline{\mathbf{w}}_{\mathscr{T}}\right\|_{L^{2}(K)}^{2}+\sum_{\gamma \in \mathcal{F}_{K}} h_{K}\left\|\llbracket \mathcal{R}_{\gamma}^{\mathrm{ad}} \rrbracket\right\|_{\boldsymbol{L}^{2}(\gamma)}^{2}\right. \\
& \left.+h_{K}^{2}\left(\left\|\boldsymbol{\mathcal { R }}_{K}^{\mathrm{ad}}\right\|_{\boldsymbol{L}^{2}(K)}^{2}+\left\|\mathbf{o s c}_{K}^{\mathrm{ad}}\right\|_{\boldsymbol{L}^{2}(K)}^{2}\right)\right)
\end{aligned}
$$

6.2. Residual-based a posteriori error estimators. From (6.23) and (6.24) the following result follows.

Theorem 6.3. Let

$$
\begin{aligned}
\tilde{\eta}_{\mathbf{y}, K}^{2}=\tilde{\eta}_{\mathrm{p}, K}^{2}= & \left\|\nabla \cdot \overline{\mathbf{y}}_{\mathscr{T}}\right\|_{L^{2}(K)}^{2}+\sum_{\gamma \in \mathcal{F}_{K}} h_{K}\left\|\llbracket \mathcal{R}_{\gamma}^{\mathrm{st}} \rrbracket\right\|_{\boldsymbol{L}^{2}(\gamma)}^{2} \\
& +h_{K}^{2}\left(\left\|\boldsymbol{\mathcal { R }}_{K}^{\mathrm{st}}\right\|_{\boldsymbol{L}^{2}(K)}^{2}+\left\|\mathbf{o s c}_{K}^{\mathrm{st}}\right\|_{\boldsymbol{L}^{2}(K)}^{2}\right),
\end{aligned}
$$




$$
\begin{aligned}
\tilde{\eta}_{\mathbf{w}, K}^{2}=\tilde{\eta}_{\mathbf{q}, K}^{2}= & \left\|\nabla \cdot \overline{\mathbf{w}}_{\mathscr{T}}\right\|_{L^{2}(K)}^{2}+\sum_{\gamma \in \mathcal{F}_{K}} h_{K}\left\|\llbracket \mathcal{R}_{\gamma}^{\mathrm{ad}} \rrbracket\right\|_{\boldsymbol{L}^{2}(\gamma)}^{2} \\
& +h_{K}^{2}\left(\left\|\boldsymbol{\mathcal { R }}_{K}^{\mathrm{ad}}\right\|_{\boldsymbol{L}^{2}(K)}^{2}+\left\|\mathbf{o s c}_{K}^{\mathrm{ad}}\right\|_{\boldsymbol{L}^{2}(K)}^{2}\right),
\end{aligned}
$$

$\eta_{\mathbf{y}, K}=C \tilde{\eta}_{\mathbf{y}, K}, \eta_{\mathbf{p}, K}=C \tilde{\eta}_{\mathbf{p}, K}, \eta_{\mathbf{w}, K}=C \tilde{\eta}_{\mathbf{w}, K}, \eta_{\mathbf{q}, K}=C \tilde{\eta}_{\mathbf{q}, K}$,

$$
\eta_{\mathbf{y}}=\eta_{\mathbf{p}}=\sum_{K \in \mathscr{T}} \eta_{\mathbf{y}, K}^{2}=\sum_{K \in \mathscr{T}} \eta_{\mathbf{p}, K}^{2}, \quad \eta_{\mathbf{w}}=\eta_{\mathbf{q}}=\sum_{K \in \mathscr{T}} \eta_{\mathbf{w}, K}^{2}=\sum_{K \in \mathscr{T}} \eta_{\mathbf{q}, K}^{2},
$$

and $\hat{\mathscr{T}}_{K}=\hat{\mathcal{F}}_{K}=\{K\}$, where $C$ is a positive constant that is independent of the size of the elements in the mesh. Then Assumptions 1, 2, 3, 4, 5 and 7 hold.

7. Numerical examples. We conduct a series of numerical examples that illustrate the performance of the devised a posteriori error estimators. These have been carried out with the help of a code that we implemented using C++. All matrices have been assembled exactly and the global linear systems were solved using the multifrontal massively parallel sparse direct solver (MUMPS) [7, 8].

For a given partition $\mathscr{T}$ we seek $\left(\overline{\mathbf{y}}_{\mathscr{T}}, \overline{\mathbf{p}}_{\mathscr{T}}, \overline{\mathbf{w}}_{\mathscr{T}}, \overline{\mathbf{q}}_{\mathscr{T}}, \overline{\mathbf{u}}_{\mathscr{T}}\right) \in \mathbf{V}(\mathscr{T}) \times Q(\mathscr{T}) \times \mathbf{V}(\mathscr{T}) \times$ $Q(\mathscr{T}) \times \mathbf{U}_{a d}(\mathscr{T})$ that solves the discrete optimality system (4.1) using the approximation method described in Section 6 with $\tau_{K}=h_{K}^{2}$ for all $K \in \mathscr{T}$ and $\tau_{\gamma}=1$ for all $\gamma \in \mathcal{F}_{I}$. We considered $\vartheta=1$ and $\varrho=1$. The number of degrees of freedom Ndof $=2 d N_{v}+(d+2) N_{e}$, where $N_{v}$ is the number of vertices in the mesh and $N_{e}$ is the number of elements in the mesh.

We solve the ensuing nonlinear system of equations using a Newton-type primaldual active set strategy [44, §2.12.4]; see also [27]. Once a discrete solution is obtained, we calculate the local error indicators, in order to drive an adaptive mesh refinement procedure, and the global error estimator, in order to assess the accuracy of the approximation. The particular global error estimator and local error indicators that we use depends on the dimension $d$ of the domain as follows:

- when $d=2$, we compute $\Upsilon$ from Theorem 5.1 and $\Upsilon_{K}$ from Theorem 5.2, with the aid of Theorems 6.1 and 6.2 ;

- when $d=3$, we compute $\tilde{\Upsilon}$ and $\tilde{\Upsilon}_{K}$ from Corollary 5.4, with the aid of Theorem 6.3.

These local error indicators are used to drive the adaptive procedures described in Algorithms 7.1 and 7.2.

Algorithm 7.1 Adaptive Primal-Dual Active Set Algorithm for $d=2$. Input: An initial mesh $\mathscr{T}$ and data $\vartheta, \mathbf{a}, \mathbf{b}, \varepsilon, \mathbf{c}, \kappa, \mathbf{y}_{\Omega}$ and $\mathbf{f}$.

1: Compute $\left(\overline{\mathbf{y}}_{\mathscr{T}}, \overline{\mathbf{p}}_{\mathscr{T}}, \overline{\mathbf{w}}_{\mathscr{T}}, \overline{\mathbf{q}}_{\mathscr{T}}, \overline{\mathbf{u}}_{\mathscr{T}}\right)$ that solves (4.1) using the active set strategy of $[44, \S 2.12 .4]$.

2: With the aid of Theorems 6.1 and 6.2, compute the local error indicators $\Upsilon_{K}$, given in Theorem 5.2, for each $K \in \mathscr{T}$, and the error estimator $\Upsilon$, given in Theorem 5.1.

3: Mark an element $K \in \mathscr{T}$ for refinement if $\Upsilon_{K}^{2} \geq N_{e}^{-1} \sum_{K^{\prime} \in \mathscr{T}} \Upsilon_{K^{\prime}}^{2}$.

4: Refine the mesh $\mathscr{T}$ using a longest edge bisection algorithm and return to step 1.

7.1. Two dimensional examples. We perform two dimensional examples on polygonal domains for which the value of a $\beta$ satisfying (2.14) is known. To accomplish 
Algorithm 7.2 Adaptive Primal-Dual Active Set Algorithm for $d=3$. Input: An initial mesh $\mathscr{T}$ and data $\vartheta, \mathbf{a}, \mathbf{b}, \varepsilon, \mathbf{c}, \kappa, \mathbf{y}_{\Omega}$ and $\mathbf{f}$.

1: Compute $\left(\overline{\mathbf{y}}_{\mathscr{T}}, \overline{\mathrm{p}}_{\mathscr{T}}, \overline{\mathbf{w}}_{\mathscr{T}}, \overline{\mathrm{q}}_{\mathscr{T}}, \overline{\mathbf{u}}_{\mathscr{T}}\right)$ that solves (4.1) using the active set strategy of $[44, \S 2.12 .4]$.

2: With the aid of Theorem 6.3, compute the local error indicators $\tilde{\Upsilon}_{K}$, for each $K \in \mathscr{T}$, and the error estimator $\tilde{\Upsilon}$, given in Corollary 5.4.

3: Mark an element $K \in \mathscr{T}$ for refinement if $\tilde{\Upsilon}_{K}^{2} \geq N_{e}^{-1} \sum_{K^{\prime} \in \mathscr{T}} \tilde{\Upsilon}_{K^{\prime}}^{2}$.

4: Refine the mesh $\mathscr{T}$ using a longest edge bisection algorithm and return to step 1.

this task we used the adaptive procedure described in Algorithm 7.1. We note that the involved estimator $\Upsilon$ provides a guaranteed upper bound on $\left\|\left(\mathbf{e}_{\mathbf{y}}, e_{\mathrm{p}}, \mathbf{e}_{\mathbf{w}}, \mathrm{e}_{\mathbf{q}}, \mathbf{e}_{\mathbf{u}}\right)\right\|_{\Omega}$. A sequence of adaptively refined meshes was generated from the initial meshes shown in Figure 7.1.

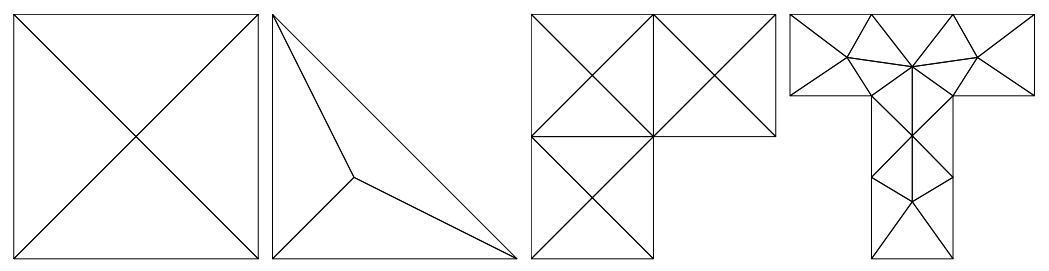

Fig. 7.1: The initial meshes used for Examples 7.1, 7.2, 7.3 and 7.4.

Example 7.1. We consider the square domain $\Omega=(0,1)^{2}$. From [41] we have that (2.14) holds with $\beta=\sin (\pi / 8)$. We took $\varepsilon=1, \mathbf{c}\left(x_{1}, x_{2}\right)=\left(x_{2},-x_{1}\right), \kappa=1$, $\mathbf{a}=(-0.5,-0.5)$ and $\mathbf{b}=(0.5,0.5)$. The data $\mathbf{f}$ and $\mathbf{y}_{\Omega}$ were chosen to be such that

$$
\begin{array}{cl}
\overline{\mathbf{y}}\left(x_{1}, x_{2}\right)=\operatorname{curl}\left(\left(x_{1}\left(1-x_{1}\right) x_{2}\left(1-x_{2}\right)\right)^{2}\right), & \overline{\mathbf{p}}\left(x_{1}, x_{2}\right)=\cos \left(2 \pi x_{1}\right) \cos \left(2 \pi x_{2}\right), \\
\overline{\mathbf{w}}\left(x_{1}, x_{2}\right)=\operatorname{curl}\left(\left(\sin \left(2 \pi x_{1}\right) \sin \left(2 \pi x_{2}\right)\right)^{2}\right), & \overline{\mathbf{q}}\left(x_{1}, x_{2}\right)=\sin \left(2 \pi x_{1}\right) \sin \left(2 \pi x_{2}\right) .
\end{array}
$$

The results are shown in Figures 7.2 and 7.3. We observe that the estimator $\Upsilon$ and the error $\left\|\left(\mathbf{e}_{\mathbf{y}}, e_{\mathbf{p}}, \mathbf{e}_{\mathbf{w}}, e_{\mathbf{q}}, \mathbf{e}_{\mathbf{u}}\right)\right\|_{\Omega}$ are decreasing at the optimal rate.

Example 7.2. We consider the triangular domain $\Omega=\left\{\left(x_{1}, x_{2}\right): x_{1}>0, x_{2}>\right.$ $\left.0, x_{1}+x_{2}<1\right\}$. From [41] we have that (2.14) holds with $\beta=\sin (\pi / 16)$. We took $\varepsilon=0.01, \mathbf{c}=(0,0), \kappa=1, \mathbf{a}=(0,0)$ and $\mathbf{b}=(0.1,0.1)$. The data $\mathbf{f}$ and $\mathbf{y}_{\Omega}$ were chosen to be such that

$$
\begin{gathered}
\overline{\mathbf{y}}\left(x_{1}, x_{2}\right)=\operatorname{curl}\left(x_{1} x_{2}^{2}\left(1-x_{1}-x_{2}\right)^{2}\left(1-x_{1}-\frac{\exp \left(-100 x_{1}\right)-\exp (-100)}{1-\exp (-100)}\right)\right), \\
\overline{\mathrm{p}}\left(x_{1}, x_{2}\right)=\cos \left(2 \pi x_{2}\right) / 1024, \\
\overline{\mathbf{w}}\left(x_{1}, x_{2}\right)=\operatorname{curl}\left(x_{1}^{2} x_{2}\left(1-x_{1}-x_{2}\right)^{2}\left(1-x_{2}-\frac{\exp \left(-100 x_{2}\right)-\exp (-100)}{1-\exp (-100)}\right)\right),
\end{gathered}
$$

and

$$
\overline{\mathrm{q}}\left(x_{1}, x_{2}\right)=\cos \left(2 \pi x_{1}\right) / 1024 .
$$



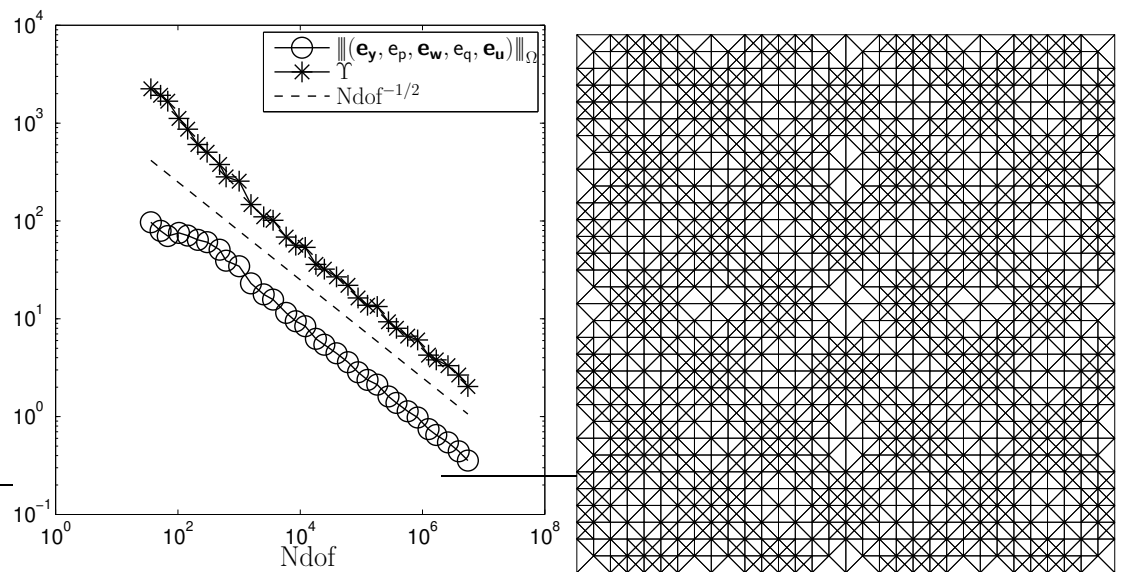

Fig. 7.2: Example 7.1: The error $\left\|\left(\mathbf{e}_{\mathbf{y}}, \mathbf{e}_{\mathrm{p}}, \mathbf{e}_{\mathbf{w}}, e_{\mathbf{q}}, \mathbf{e}_{\mathbf{u}}\right)\right\|_{\Omega}$ and estimator $\Upsilon$ (left) and the 16 th adaptively refined mesh (right).

The results are shown in Figures 7.4 and 7.5. We observe that, once the mesh has been sufficiently refined, the error $\left\|\left(\mathbf{e}_{\mathbf{y}}, e_{\mathrm{p}}, \mathbf{e}_{\mathbf{w}}, e_{\mathbf{q}}, \mathbf{e}_{\mathbf{u}}\right)\right\|_{\Omega}$ and the estimator $\Upsilon$ decrease at the optimal rate. We also observe that more refinement has been performed in the regions where the solution has boundary layers.

Example 7.3. We consider the L-shaped domain $\Omega=(-1,1)^{2} \backslash([0,1) \times(-1,0])$. From [41] we have that (2.14) holds with $\beta=0.1601$. We took $\varepsilon=1, \mathbf{c}=(0,0)$, $\kappa=0, \mathbf{a}=(0,0), \mathbf{b}=(1,1), \mathbf{f}=(1,1)$ and $\mathbf{y}_{\Omega}\left(x_{1}, x_{2}\right)=\left(x_{2},-x_{1}\right)$. The results are shown in Figures 7.6 and 7.7. We observe that the estimator $\Upsilon$ decreases at the optimal rate and that more refinement is being performed in regions close to the reentrant corner. The true solution to this problem is unknown and hence we cannot compute $\left\|\left(\mathbf{e}_{\mathbf{y}}, \mathbf{e}_{\mathbf{p}}, \mathbf{e}_{\mathbf{w}}, \mathbf{e}_{\mathbf{q}}, \mathbf{e}_{\mathbf{u}}\right)\right\|_{\Omega}$. However, from Theorem 5.1 we know that $\left\|\left(\mathbf{e}_{\mathbf{y}}, e_{\mathrm{p}}, \mathbf{e}_{\mathbf{w}}, e_{\mathrm{q}}, \mathbf{e}_{\mathbf{u}}\right)\right\|_{\Omega} \leq \Upsilon$.

Example 7.4. We considered the same problem as in the previous example with the exception that we took the domain to be the T-shaped domain $\Omega=((-1.5,1.5) \times$ $(0,1)) \cup((-0.5,0.5) \times(-2,0])$ on which we have that $(2.14)$ holds with $\beta=0.1076$ from [41]. The results are shown in Figures 7.8 and 7.9. Similar observations to those made about the previous example can be made.

7.2. Three dimensional examples. Unfortunately, we are not aware of any polyhedral domains for which the value of a $\beta$ satisfying (2.14) is known. Hence, when the domain is three dimensional, the estimator $\Upsilon$ from Theorem 5.1 and the local error indicators $\Upsilon_{K}$ from Theorem 5.2 are not computable. Consequently, we performed numerical examples by following the adaptive procedure described in Algorithm 7.2. A sequence of adaptively refined meshes was generated from the initial meshes shown in Figure 7.10. We note that we have not proved that the estimator $\tilde{\Upsilon}$ provides a guaranteed upper bound on $\left\|\left(\mathbf{e}_{\mathbf{y}}, e_{\mathrm{p}}, \mathbf{e}_{\mathbf{w}}, e_{\mathbf{q}}, \mathbf{e}_{\mathbf{u}}\right)\right\|_{\Omega}$. However, from Theorem 5.4 we know that $\left\|\left(\mathbf{e}_{\mathbf{y}}, e_{\mathrm{p}}, \mathbf{e}_{\mathbf{w}}, \mathrm{e}_{\mathrm{q}}, \mathbf{e}_{\mathbf{u}}\right)\right\|_{\Omega} \lesssim \tilde{\Upsilon}$.

Example 7.5. We consider the cuboidal domain $\Omega=(0,1)^{3}$. We took $\varepsilon=1$, $\mathbf{c}\left(x_{1}, x_{2}, x_{3}\right)=\left(x_{2}-x_{3}, x_{3}-x_{1}, x_{1}-x_{2}\right), \kappa=1, \mathbf{a}=(-0.5,-0.5,-0.5)$ and $\mathbf{b}=$ 

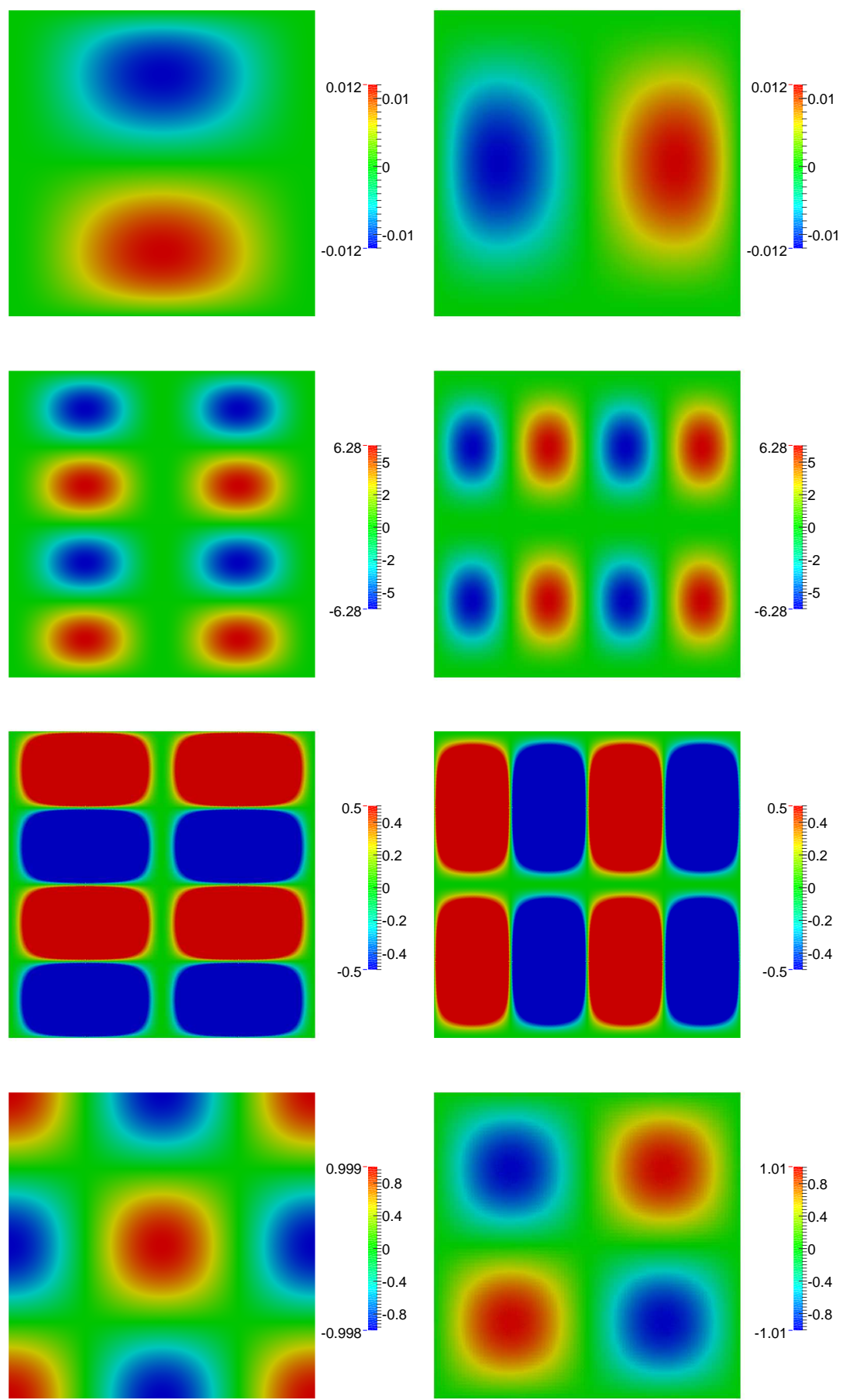

Fig. 7.3: Example 7.1: The first (left) and second (right) entries of $\overline{\mathbf{y}}_{\mathscr{T}}$ (top), $\overline{\mathbf{w}}_{\mathscr{T}}$ (second row) and $\overline{\mathbf{u}}_{\mathscr{T}}$ (third row), as well as $\overline{\mathrm{p}} \mathscr{T}$ (bottom left) and $\overline{\mathrm{q}} \mathscr{T}$ (bottom right), obtained on the final adaptively refined mesh. 

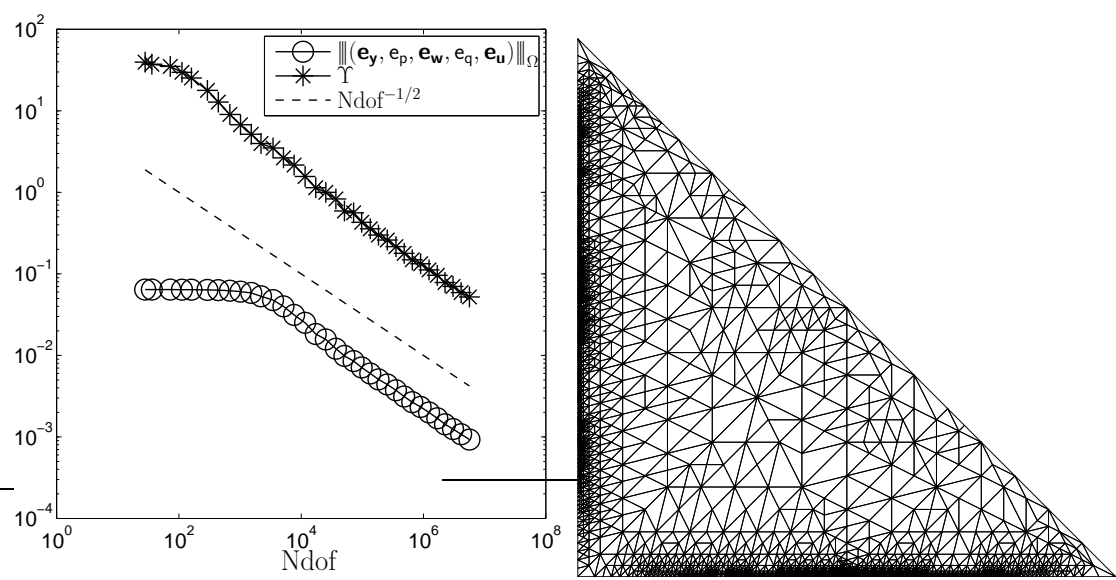

Fig. 7.4: Example 7.2: The error $\left\|\left(\mathbf{e}_{\mathbf{y}}, \mathbf{e}_{\mathbf{p}}, \mathbf{e}_{\mathbf{w}}, \mathbf{e}_{\mathbf{q}}, \mathbf{e}_{\mathbf{u}}\right)\right\|_{\Omega}$ and estimator $\Upsilon$ (left) and the 16 th adaptively refined mesh (right).

$(0.5,0.5,0.5)$. The data $\mathbf{f}$ and $\mathbf{y}_{\Omega}$ were chosen to be such that

$$
\begin{gathered}
\overline{\mathbf{y}}\left(x_{1}, x_{2}, x_{3}\right)=\operatorname{curl}\left(\left(x_{1}\left(1-x_{1}\right) x_{2}\left(1-x_{2}\right) x_{3}\left(1-x_{3}\right)\right)^{2}\right), \overline{\mathrm{p}}\left(x_{1}, x_{2}, x_{3}\right)=\cos \left(2 \pi x_{3}\right), \\
\overline{\mathbf{w}}\left(x_{1}, x_{2}, x_{3}\right)=\operatorname{curl}\left(\left(\sin \left(2 \pi x_{1}\right) \sin \left(2 \pi x_{2}\right) \sin \left(2 \pi x_{3}\right)\right)^{2}\right), \overline{\mathbf{q}}\left(x_{1}, x_{2}, x_{3}\right)=\sin \left(2 \pi x_{3}\right) .
\end{gathered}
$$

The results are shown in Figures 7.11 and 7.12. We observe that the estimator $\tilde{\Upsilon}$ and the error $\left\|\left(\mathbf{e}_{\mathbf{y}}, e_{\mathbf{p}}, \mathbf{e}_{\mathbf{w}}, e_{\mathbf{q}}, \mathbf{e}_{\mathbf{u}}\right)\right\|_{\Omega}$ are decreasing at the optimal rate.

Example 7.6. We consider the tetrahedral domain $\Omega=\left\{\left(x_{1}, x_{2}, x_{3}\right): x_{1}>0, x_{2}>\right.$ $\left.0, x_{3}>0, x_{1}+x_{2}+x_{3}<1\right\}$. We took $\varepsilon=0.01, \mathbf{c}=(1,1,1), \kappa=0, \mathbf{a}=(0,0,0)$ and $\mathbf{b}=(0.1,0.1,0.1)$. The data $\mathbf{f}$ and $\mathbf{y}_{\Omega}$ were chosen to be such that

$$
\begin{aligned}
& \overline{\mathbf{y}}\left(x_{1}, x_{2}, x_{3}\right)=\operatorname{curl}\left(x_{1} x_{2}^{2} \chi\left(1-x_{1}-\frac{\exp \left(-100 x_{1}\right)-\exp (-100)}{1-\exp (-100)}\right)\right), \\
& \overline{\mathrm{p}}\left(x_{1}, x_{2}, x_{3}\right)=\left(\cos \left(2 \pi x_{3}\right)-3 /\left(2 \pi^{2}\right)\right) / 1024 \\
& \overline{\mathbf{w}}\left(x_{1}, x_{2}, x_{3}\right)=\operatorname{curl}\left(x_{1}^{2} x_{2} \chi\left(1-x_{2}-\frac{\exp \left(-100 x_{2}\right)-\exp (-100)}{1-\exp (-100)}\right)\right),
\end{aligned}
$$

and

$$
\overline{\mathrm{q}}\left(x_{1}, x_{2}, x_{3}\right)=\left(\sin \left(2 \pi x_{3}\right)-3 /(2 \pi)\right) / 1024,
$$

where $\chi=x_{3}^{2}\left(1-x_{1}-x_{2}-x_{3}\right)^{2}$. The results are shown in Figures 7.13 and 7.14. We observe that, once the mesh has been sufficiently refined, the error $\left\|\left(\mathbf{e}_{\mathbf{y}}, \mathrm{e}_{\mathrm{p}}, \mathbf{e}_{\mathbf{w}}, \mathrm{e}_{\mathbf{q}}, \mathbf{e}_{\mathbf{u}}\right)\right\|_{\Omega}$ and the estimator $\tilde{\Upsilon}$ decrease at the optimal rate. We also observe that more refinement has been performed in the regions where the solution has boundary layers.

\section{REFERENCES}

[1] M. Ainsworth, A. Allendes, G. R. Barrenechea, and R. Rankin, On the adaptive selection of the parameter in stabilized finite element approximations, SIAM J. Numer. Anal., 51 (2013), pp. 1585-1609, https://doi.org/10.1137/110837796. 

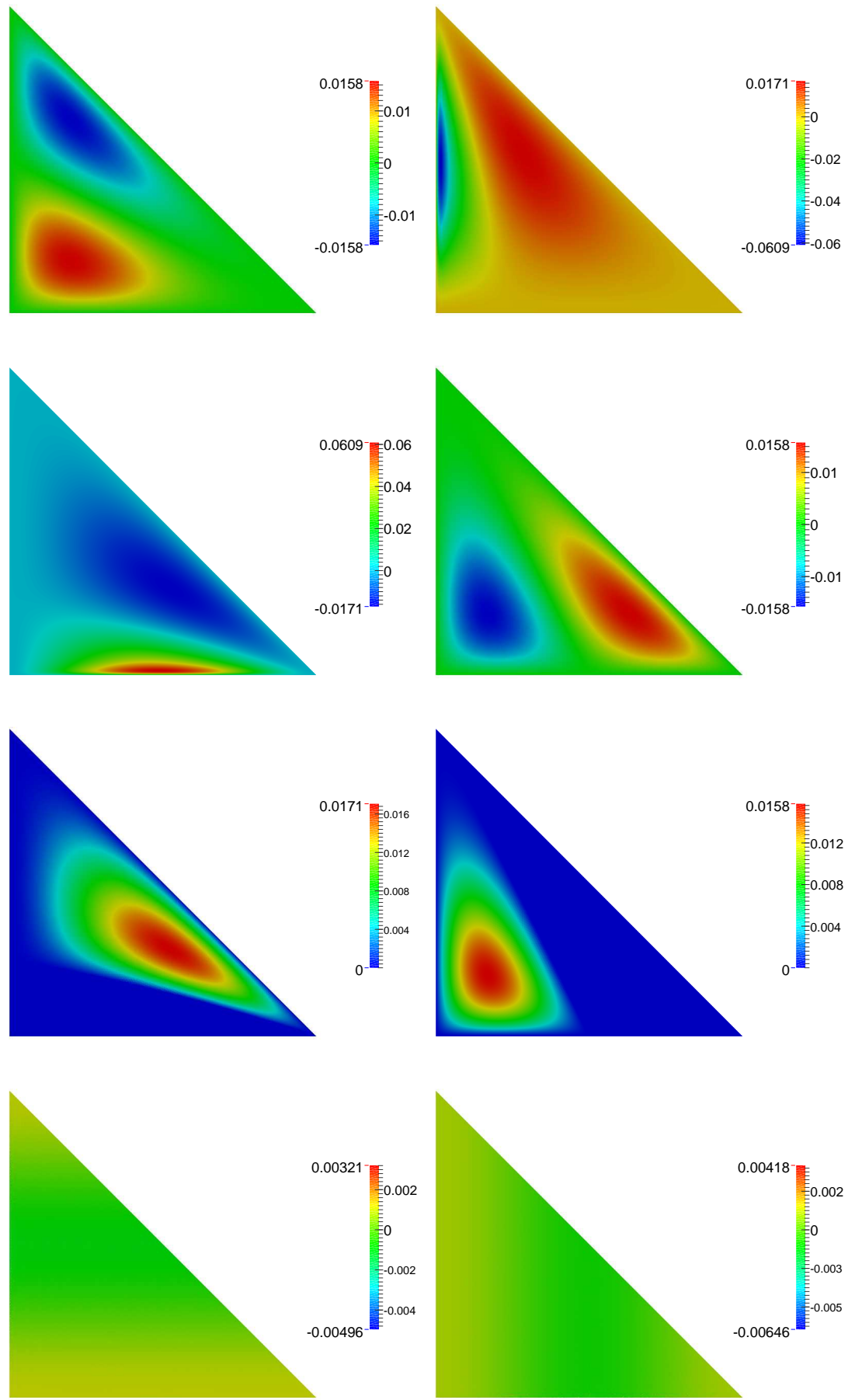

Fig. 7.5: Example 7.2: The first (left) and second (right) entries of $\overline{\mathbf{y}}_{\mathscr{T}}$ (top), $\overline{\mathbf{w}}_{\mathscr{T}}$ (second row) and $\overline{\mathbf{u}}_{\mathscr{T}}$ (third row), as well as $\overline{\mathrm{p}}_{\mathscr{T}}$ (bottom left) and $\overline{\mathrm{q}}_{\mathscr{T}}$ (bottom right), obtained on the final adaptively refined mesh. 

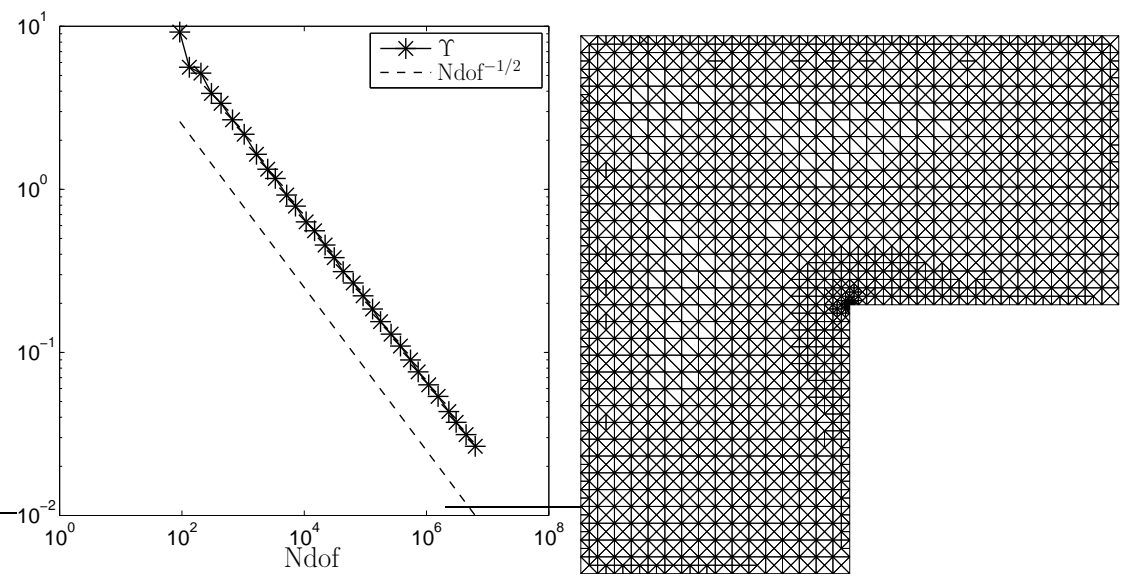

Fig. 7.6: Example 7.3: The estimator $\Upsilon$ (left) and the 14th adaptively refined mesh (right).

[2] M. Ainsworth And J. T. Oden, A posteriori error estimators for the Stokes and Oseen equations, SIAM J. Numer. Anal., 34 (1997), pp. 228-245, https://doi.org/10.1137/ S0036142994264092.

[3] M. Ainsworth and J. T. Oden, A posteriori error estimation in finite element analysis, Pure and Applied Mathematics (New York), Wiley-Interscience, New York, 2000, https://doi. org/10.1002/9781118032824.

[4] A. Allendes, G. R. Barrenechea, And R. Rankin, Fully computable error estimation of a nonlinear, positivity-preserving discretization of the convection-diffusion-reaction equation, SIAM J. Sci. Comput., 39 (2017), pp. A1903-A1927, https://doi.org/10.1137/ 16M1092763.

[5] A. Allendes, F. Durán, And R. Rankin, Error estimation for low-order adaptive finite element approximations for fluid flow problems, IMA J. Numer. Anal., 36 (2016), pp. 17151747, https://doi.org/10.1093/imanum/drv031.

[6] A. Allendes, E. Otárola, and R. Rankin, A posteriori error estimators for stabilized finite element approximations of an optimal control problem, Comput. Methods Appl. Mech. Engrg., 340 (2018), pp. 147-177.

[7] P. R. Amestoy, I. S. Duff, J.-Y. L'Excellent, And J. Koster, A fully asynchronous multifrontal solver using distributed dynamic scheduling, SIAM J. Matrix Anal. Appl., 23 (2001), pp. 15-41 (electronic), https://doi.org/10.1137/S0895479899358194, http://dx.doi.org/10. $1137 /$ S0895479899358194.

[8] P. R. Amestoy, A. Guermouche, J.-Y. L'Excellent, and S. Pralet, Hybrid scheduling for the parallel solution of linear systems, Parallel Comput., 32 (2006), pp. 136-156, https:// doi.org/10.1016/j.parco.2005.07.004, http://dx.doi.org/10.1016/j.parco.2005.07.004.

[9] R. Araya, G. R. Barrenechea, and A. Poza, An adaptive stabilized finite element method for the generalized Stokes problem, J. Comput. Appl. Math., 214 (2008), pp. 457-479, https://doi.org/10.1016/j.cam.2007.03.011.

[10] M. Bebendorf, A note on the Poincaré inequality for convex domains, Z. Anal. Anwendungen, 22 (2003), pp. 751-756, https://doi.org/10.4171/ZAA/1170.

[11] R. Becker, Mesh adaptation for stationary flow control, J. Math. Fluid Mech., 3 (2001), pp. 317-341, https://doi.org/10.1007/PL00000974.

[12] R. Becker, M. Braack, D. Meidner, R. Rannacher, and B. Vexler, Adaptive finite element methods for PDE-constrained optimal control problems, in Reactive flows, diffusion and transport, Springer, Berlin, 2007, pp. 177-205, https://doi.org/10.1007/ 978-3-540-28396-6_8.

[13] R. Becker And R. Rannacher, A feed-back approach to error control in finite element methods: basic analysis and examples, East-West J. Numer. Math., 4 (1996), pp. 237-264.

[14] P. Bochev And M. D. GunzBurger, Least-squares finite-element methods for optimization and control problems for the Stokes equations, Comput. Math. Appl., 48 (2004), pp. 1035- 

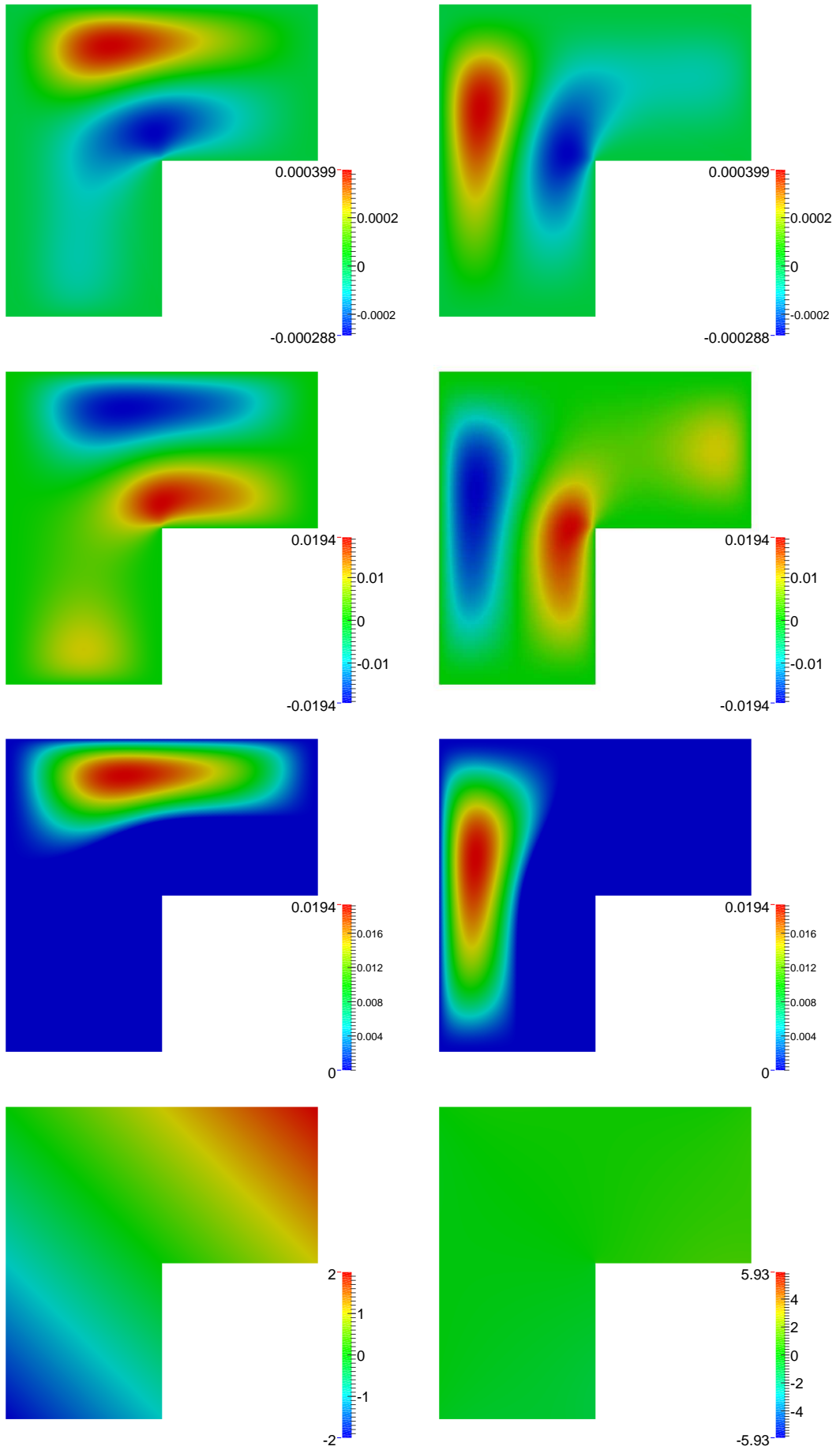

Fig. 7.7: Example 7.3: The first (left) and second (right) entries of $\overline{\mathbf{y}}_{\mathscr{T}}$ (top), $\overline{\mathbf{w}}_{\mathscr{T}}$ (second row) and $\overline{\mathbf{u}}_{\mathscr{T}}$ (third row), as well as $\overline{\mathrm{p}}_{\mathscr{T}}$ (bottom left) and $\overline{\mathrm{q}}_{\mathscr{T}}$ (bottom right), obtained on the final adaptively refined mesh. 

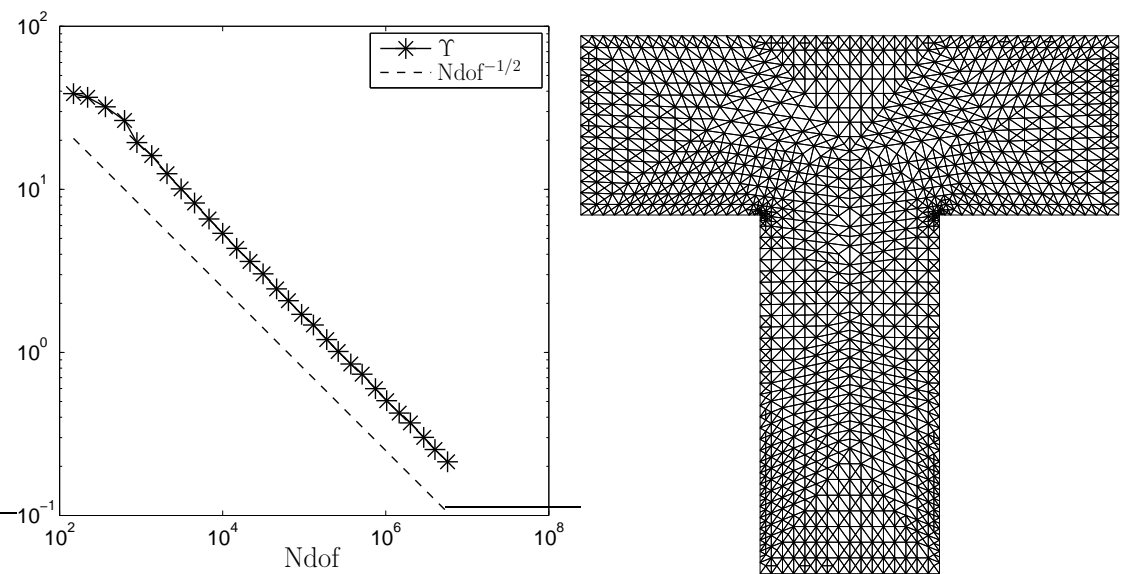

Fig. 7.8: Example 7.4: The estimator $\Upsilon$ (left) and the 12th adaptively refined mesh (right).

1057, https://doi.org/10.1016/j.camwa.2004.10.004.

[15] A. Borzì AND V. Schulz, Computational optimization of systems governed by partial differential equations, vol. 8 of Computational Science \& Engineering, Society for Industrial and Applied Mathematics (SIAM), Philadelphia, PA, 2012.

[16] M. BraACK, Optimal control in fluid mechanics by finite elements with symmetric stabilization, SIAM J. Control Optim., 48 (2009), pp. 672-687, https://doi.org/10.1137/060653494.

[17] M. Braack, E. Burman, V. John, and G. Lube, Stabilized finite element methods for the generalized oseen problem, Computer Methods in Applied Mechanics and Engineering, 196 (2007), pp. 853-866.

[18] P. G. Ciarlet, The finite element method for elliptic problems, vol. 40 of Classics in Applied Mathematics, Society for Industrial and Applied Mathematics (SIAM), Philadelphia, PA, 2002, https://doi.org/10.1137/1.9780898719208. Reprint of the 1978 original [NorthHolland, Amsterdam; MR0520174 (58 \#25001)].

[19] S. S. Collis And M. Heinkenschloss, Analysis of the streamline upwind/petrov galerkin method applied to the solution of optimal control problems, CAAM TR02-01, (2002).

[20] M. Cui And N. Yan, Residual based a posteriori error estimates for convex optimal control problems governed by Stokes-Darcy equations, Numer. Math. Theory Methods Appl., 5 (2012), pp. 602-634, https://doi.org/10.4208/nmtma.2012.m1113.

[21] J. C. De los Reyes, Numerical PDE-constrained optimization, Springer Briefs in Optimization, Springer, Cham, 2015, https://doi.org/10.1007/978-3-319-13395-9.

[22] J. C. de los Reyes, C. Meyer, And B. Vexler, Finite element error analysis for stateconstrained optimal control of the Stokes equations, Control Cybernet., 37 (2008), pp. 251284.

[23] A. Ern And J.-L. Guermond, Theory and practice of finite elements, vol. 159 of Applied Mathematical Sciences, Springer-Verlag, New York, 2004, https://doi.org/10.1007/ 978-1-4757-4355-5.

[24] V. Girault and P.-A. Raviart, Finite element methods for Navier-Stokes equations, vol. 5 of Springer Series in Computational Mathematics, Springer-Verlag, Berlin, 1986, https:// doi.org/10.1007/978-3-642-61623-5. Theory and algorithms.

[25] M. Heinkenschloss And D. Leykekhman, Local error estimates for SUPG solutions of advection-dominated elliptic linear-quadratic optimal control problems, SIAM J. Numer. Anal., 47 (2010), pp. 4607-4638, https://doi.org/10.1137/090759902.

[26] M. Hinze, R. Pinnau, M. Ulbrich, and S. Ulbrich, Optimization with PDE constraints, vol. 23 of Mathematical Modelling: Theory and Applications, Springer, New York, 2009.

[27] K. Ito And K. Kunisch, Augmented lagrangian methods for nonsmooth, convex optimization in hilbert spaces, Nonlinear Analysis: Theory, Methods \& Applications, 41 (2000), pp. 591616.

[28] K. Kohls, A. Rösch, AND K. G. SieBert, A posteriori error analysis of optimal control 

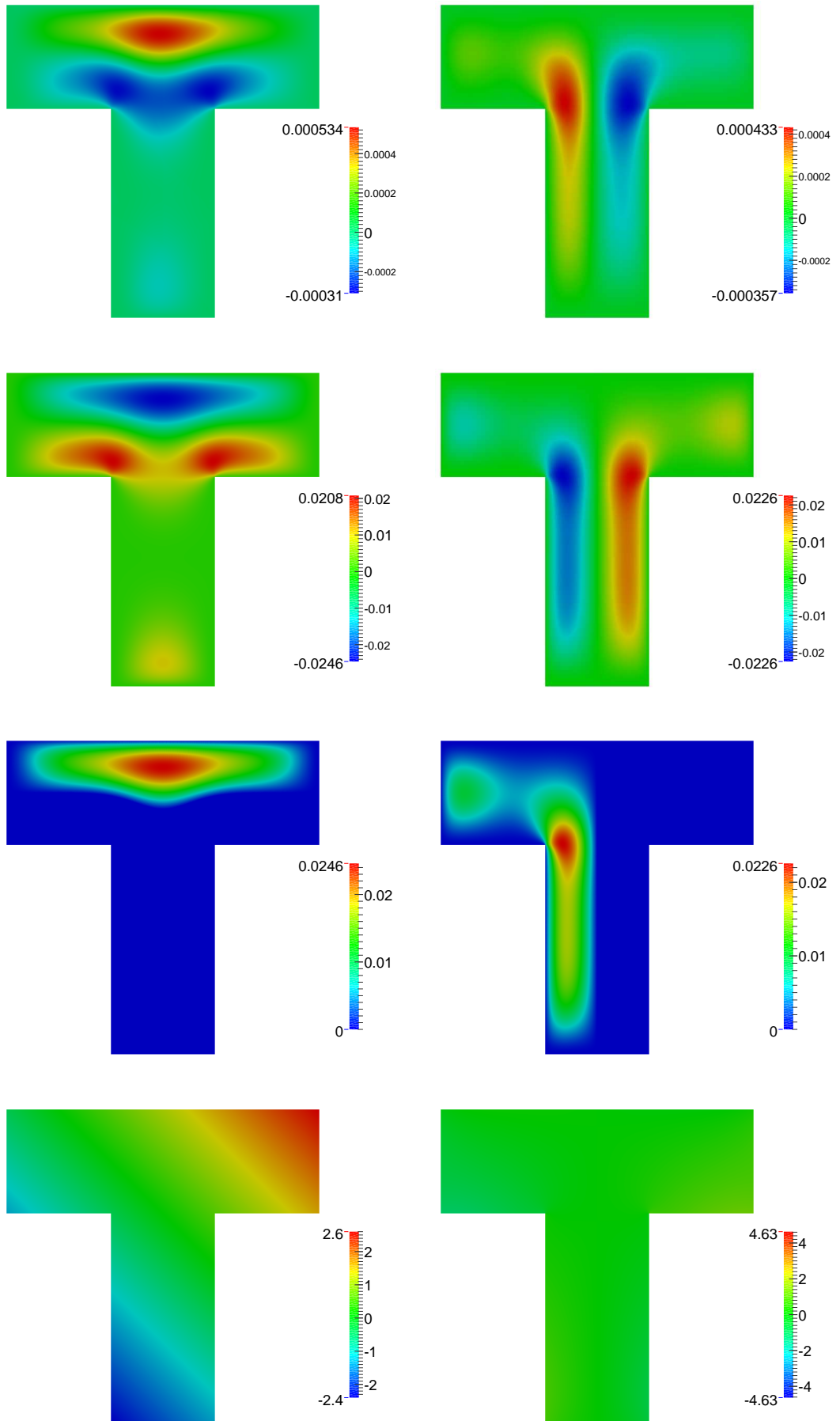

Fig. 7.9: Example 7.4: The first (left) and second (right) entries of $\overline{\mathbf{y}}_{\mathscr{T}}$ (top), $\overline{\mathbf{w}}_{\mathscr{T}}$ (second row) and $\overline{\mathbf{u}}_{\mathscr{T}}$ (third row), as well as $\overline{\mathrm{p}}_{\mathscr{T}}$ (bottom left) and $\overline{\mathrm{q}}_{\mathscr{T}}$ (bottom right), obtained on the final adaptively refined mesh. 


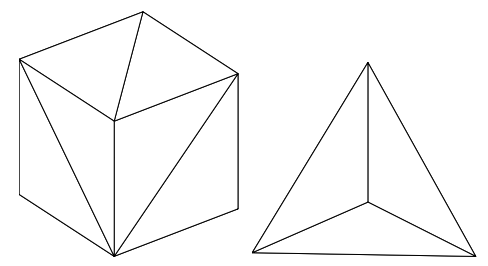

Fig. 7.10: Exterior views of the initial meshes used for Examples 7.5 and 7.6.
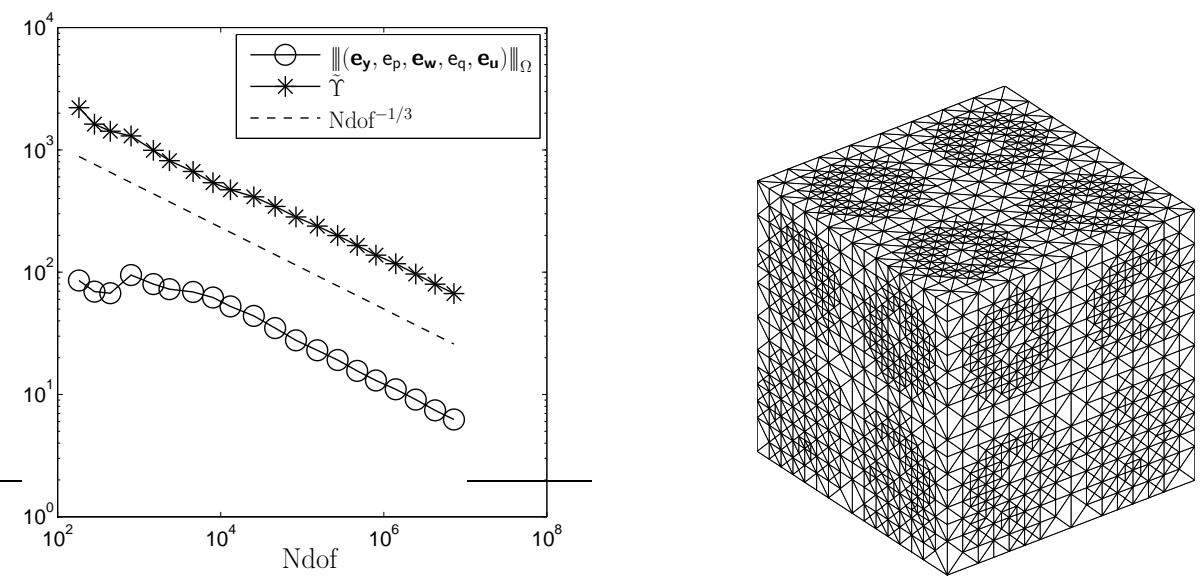

Fig. 7.11: Example 7.5: The error $\left\|\left(\mathbf{e}_{\mathbf{y}}, \mathrm{e}_{\mathrm{p}}, \mathbf{e}_{\mathbf{w}}, \mathrm{e}_{\mathbf{q}}, \mathbf{e}_{\mathbf{u}}\right)\right\|_{\Omega}$ and estimator $\tilde{\Upsilon}$ (left) and an exterior view of the 16 th adaptively refined mesh (right).

problems with control constraints, SIAM J. Control Optim., 52 (2014), pp. 1832-1861, https://doi.org/10.1137/130909251.

[29] D. LeykeKhman, Investigation of commutative properties of discontinuous Galerkin methods in PDE constrained optimal control problems, J. Sci. Comput., 53 (2012), pp. 483-511, https://doi.org/10.1007/s10915-012-9582-y.

[30] J.-L. Lions, Optimal control of systems governed by partial differential equations., Translated from the French by S. K. Mitter. Die Grundlehren der mathematischen Wissenschaften, Band 170, Springer-Verlag, New York-Berlin, 1971.

[31] H. LiU AND N. YAN, Recovery type superconvergence and a posteriori error estimates for control problems governed by Stokes equations, J. Comput. Appl. Math., 209 (2007), pp. 187-207, https://doi.org/10.1016/j.cam.2006.10.083.

[32] W. LiU AND N. YAN, A posteriori error estimates for control problems governed by Stokes equations, SIAM J. Numer. Anal., 40 (2002), pp. 1850-1869, https://doi.org/10.1137/ S0036142901384009.

[33] S. G. Mikhlin, Constants in some inequalities of analysis, A Wiley-Interscience Publication, John Wiley \& Sons, Ltd., Chichester, 1986. Translated from the Russian by Reinhard Lehmann.

[34] S. NicAise AND D. Sirch, Optimal control of the Stokes equations: conforming and nonconforming finite element methods under reduced regularity, Comput. Optim. Appl., 49 (2011), pp. 567-600, https://doi.org/10.1007/s10589-009-9305-y.

[35] H. NiU, L. YUAN, AND D. YANG, Adaptive finite element method for an optimal control problem of Stokes flow with $L^{2}$-norm state constraint, Internat. J. Numer. Methods Fluids, 69 (2012), pp. 534-549, https://doi.org/10.1002/fld.2572.

[36] R. H. Nochetto, K. G. Siebert, ANd A. Veeser, Theory of adaptive finite element methods: an introduction, in Multiscale, nonlinear and adaptive approximation, Springer, Berlin, 2009, pp. 409-542, https://doi.org/10.1007/978-3-642-03413-8_12. 



Fig. 7.12: Example 7.5: Slices at $x_{3}=0.75$ of the first (left), second (middle) and third (right) entries of $\overline{\mathbf{y}}_{\mathscr{T}}$ (top), $\overline{\mathbf{w}}_{\mathscr{T}}$ (second row) and $\overline{\mathbf{u}}_{\mathscr{T}}$ (third row), as well as $\overline{\mathbf{p}}_{\mathscr{T}}$ (bottom left) and $\overline{\mathrm{q}}_{\mathscr{T}}$ (bottom right), obtained on the final adaptively refined mesh.

[37] L. E. Payne and H. F. Weinberger, An optimal Poincaré inequality for convex domains, Arch. Rational Mech. Anal., 5 (1960), pp. 286-292 (1960).

[38] H.-G. Roos, M. STynes, and L. TOBiska, Robust numerical methods for singularly perturbed differential equations, vol. 24 of Springer Series in Computational Mathematics, SpringerVerlag, Berlin, second ed., 2008. Convection-diffusion-reaction and flow problems.

[39] A. Rösch ANd B. VeXler, Optimal control of the Stokes equations: a priori error analysis for finite element discretization with postprocessing, SIAM J. Numer. Anal., 44 (2006), pp. 1903-1920, https://doi.org/10.1137/050637364.

[40] S. RYu, H.-C. LEE, AND S. D. KIM, First-order system least-squares methods for an optimal control problem by the Stokes flow, SIAM J. Numer. Anal., 47 (2009), pp. 1524-1545, https://doi.org/10.1137/070701157.

[41] G. Stoyan, Towards discrete Velte decompositions and narrow bounds for inf-sup constants, Comput. Math. Appl., 38 (1999), pp. 243-261, https://doi.org/10.1016/ 

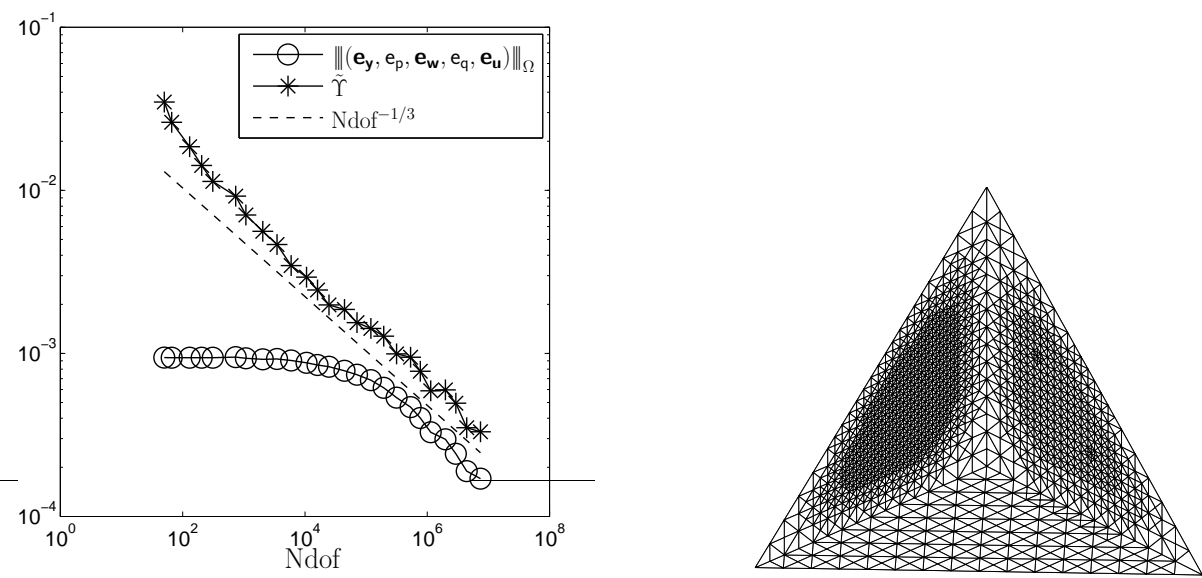

Fig. 7.13: Example 7.6: The error $\left\|\left(\mathbf{e}_{\mathbf{y}}, \mathrm{e}_{\mathbf{p}}, \mathbf{e}_{\mathbf{w}}, \mathrm{e}_{\mathbf{q}}, \mathbf{e}_{\mathbf{u}}\right)\right\|_{\Omega}$ and estimator $\tilde{\Upsilon}$ (left) and an exterior view of the 16th adaptively refined mesh (right).

S0898-1221(99)00254-0.

[42] S. TAKaCS, A robust all-at-once multigrid method for the Stokes control problem, Numer. Math., 130 (2015), pp. 517-540, https://doi.org/10.1007/s00211-014-0674-5.

[43] L. Tobiska and R. Verfürth, Robust a posteriori error estimates for stabilized finite element methods, IMA J. Numer. Anal., 35 (2015), pp. 1652-1671, https://doi.org/10.1093/ imanum/dru060.

[44] F. TröLtzsch, Optimal control of partial differential equations, vol. 112 of Graduate Studies in Mathematics, American Mathematical Society, Providence, RI, 2010, https://doi.org/ $10.1090 / \mathrm{gsm} / 112$. Theory, methods and applications, Translated from the 2005 German original by Jürgen Sprekels.

[45] R. VERFüRTH, A posteriori error estimators for the Stokes equations, Numer. Math., 55 (1989), pp. 309-325, https://doi.org/10.1007/BF01390056.

[46] R. Verfürth, A posteriori error estimation techniques for finite element methods, Numerical Mathematics and Scientific Computation, Oxford University Press, Oxford, 2013, https:// doi.org/10.1093/acprof:oso/9780199679423.001.0001.

[47] J. WANG, Y. WANG, AND X. YE, A posteriori error estimate for stabilized finite element methods for the Stokes equations, Int. J. Numer. Anal. Model., 9 (2012), pp. 1-16. 

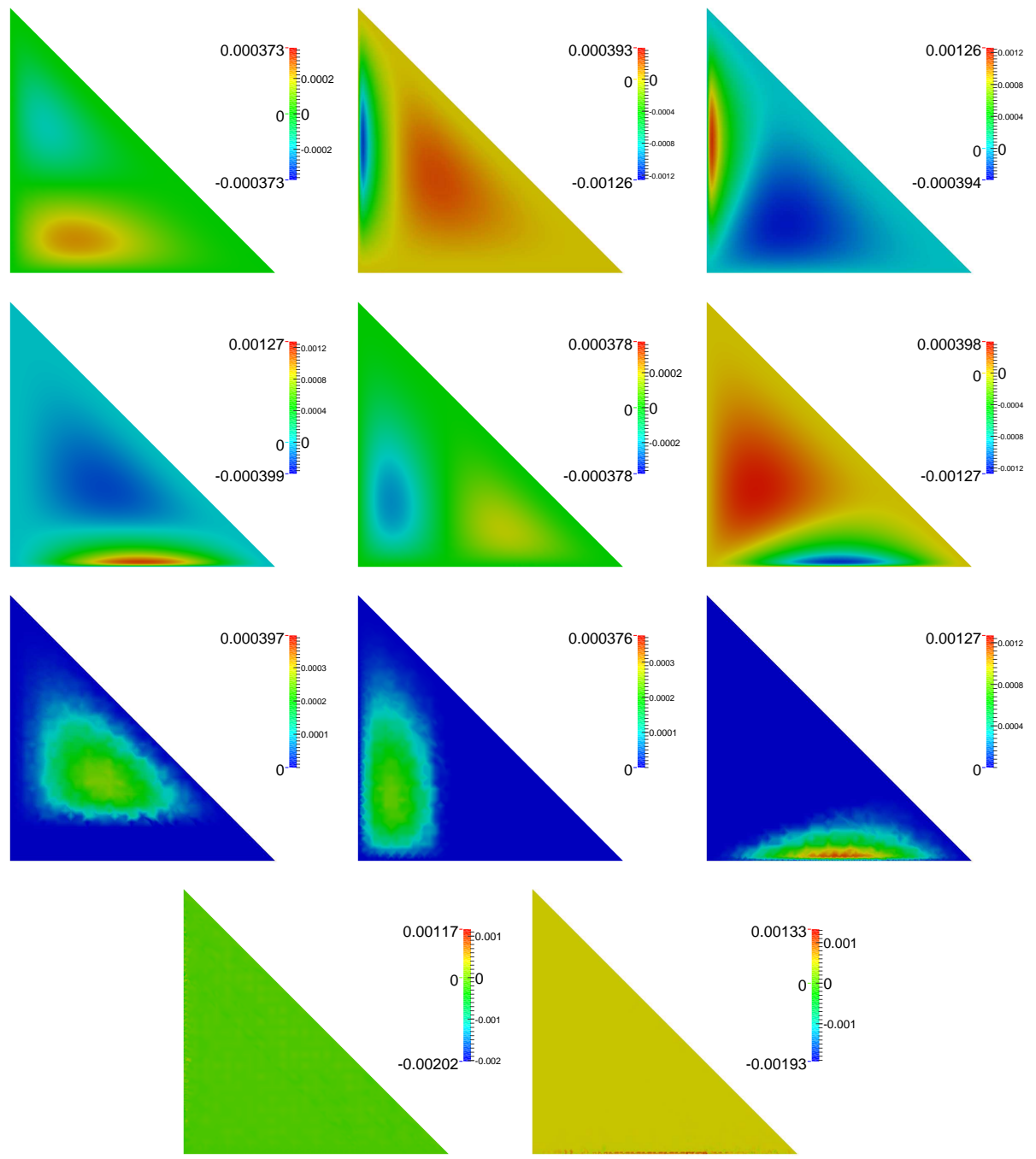

Fig. 7.14: Example 7.6: Slices at $x_{3}=0.25$ of the first (left), second (middle) and third (right) entries of $\overline{\mathbf{y}}_{\mathscr{T}}$ (top), $\overline{\mathbf{w}}_{\mathscr{T}}$ (second row) and $\overline{\mathbf{u}}_{\mathscr{T}}$ (third row), as well as $\overline{\mathbf{p}} \mathscr{T}$ (bottom left) and $\overline{\mathrm{q}}_{\mathscr{T}}$ (bottom right), obtained on the final adaptively refined mesh. 